\title{
MOLECULAR HYDROGEN AFFORDS NEUROPROTECTION IN A NEW TRANSLATIONAL PIGLET MODEL OF HYPOXIC-ISCHEMIC ENCEPHALOPATHY
}

\author{
Ph.D. Thesis
}

János Németh, MD

Szeged

2017 
MOLECULAR HYDROGEN AFFORDS NEUROPROTECTION IN A NEW TRANSLATIONAL PIGLET MODEL OF HYPOXIC-ISCHEMIC ENCEPHALOPATHY

by

János Németh, MD

A Thesis for the Degree of DOCTOR OF PHYLOSOPHY

(Ph.D.)

In the Department of Physiology

School of Medicine, University of Szeged

Consultant: Ferenc Domoki, MD, Ph.D.

Doctoral School of Theoretical Medicine

2017

Szeged 
PUBLICATIONS RELATED TO THE SUBJECT OF THE THESIS

I. Domoki F, Zolei-Szenasi D, Olah O, Toth-Szuki V, Nemeth J, Hopp B, Bari F, Smausz T (2014) Comparison of cerebrocortical microvascular effects of different hypoxic-ischemic insults in piglets: a laser-speckle imaging study. J. Physiol. Pharmacol. 65(4): 551-8.

IF: 2.386

II. Nemeth J, Toth-Szuki V, Varga V, Kovacs V, Remzso G, Domoki F (2016) Molecular hydrogen affords neuroprotection in a translational piglet model of hypoxic-ischemic encephalopathy. J. Physiol. Pharmacol. 67(5): 677-689.

IF: 2.883 


\section{Table of contents:}

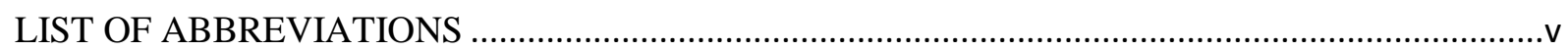

LIST OF TABLES AND FIGURES ….............................................................................................

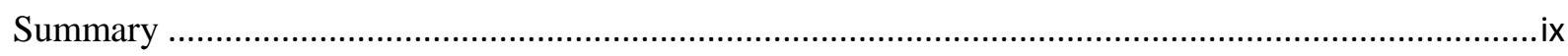

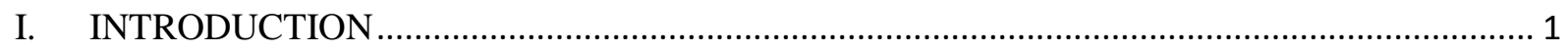

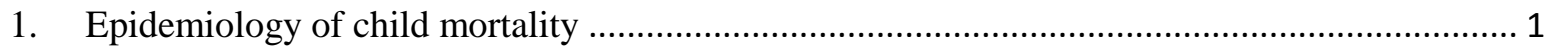

2. PA and hypoxic-ischemic encephalopathy (HIE) …............................................................. 1

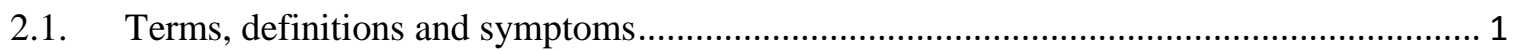

2.2. Clinical manifestations and tools for grading HIE …...................................................... 3

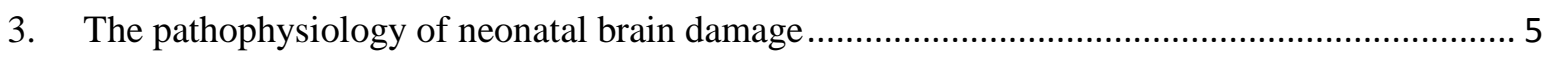

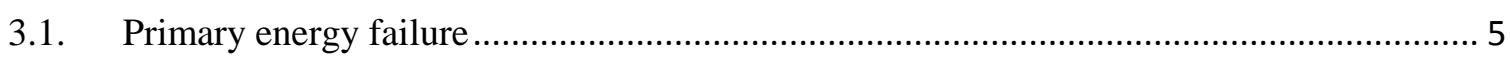

3.2. Reperfusion and secondary energy failure (SEF) .................................................... 6

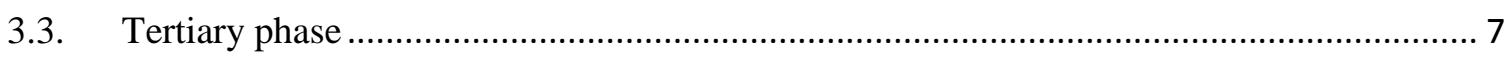

3.4. Metabolic biomarkers of brain injury ......................................................................... 7

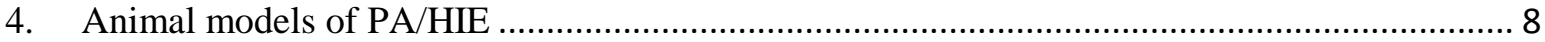

5. The newborn pig as model of the neonate …….................................................................. 9

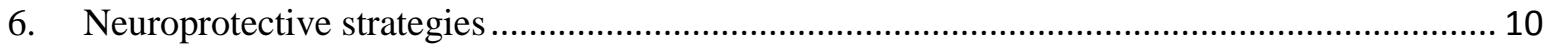

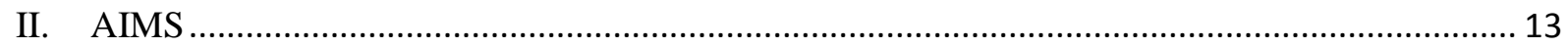

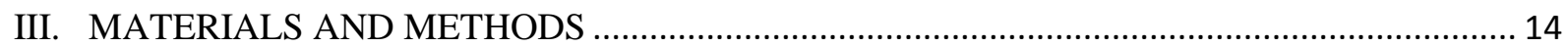

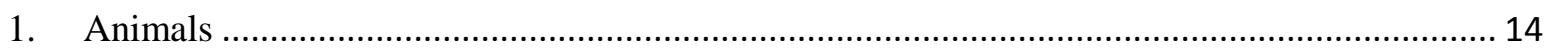

2. Laser speckle contrast imaging (LSCI) and analysis (LASCA) …....................................... 16

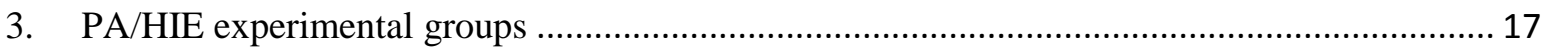

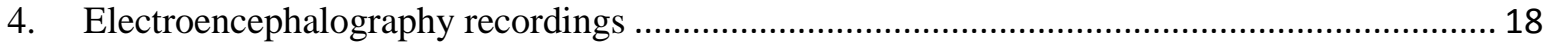

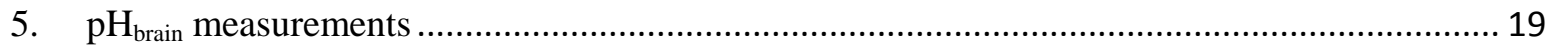

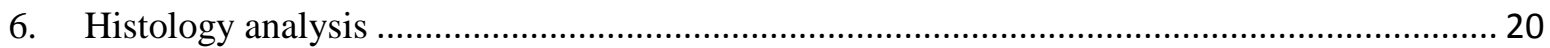

7. 8-hydroxy-2'-dezoxyguanosine (8-OHdG) immunohistochemistry ........................................ 21

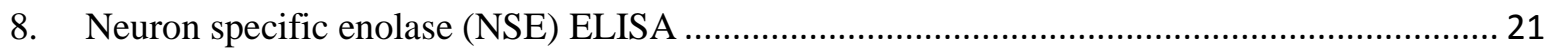

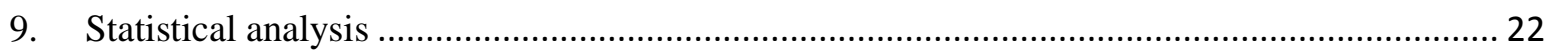

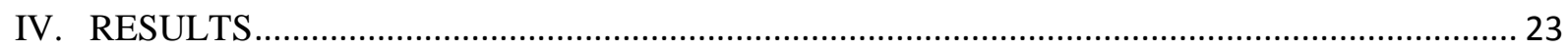

1. The effects of BCAO on CoBF velocity under different ventilation conditions........................ 23

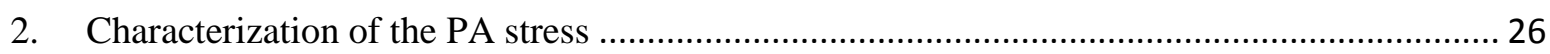

3. Characterization of the HIE development …....................................................................... 30

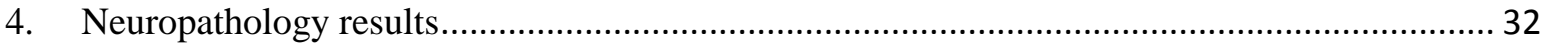




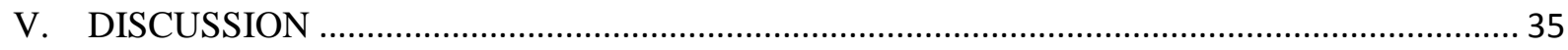

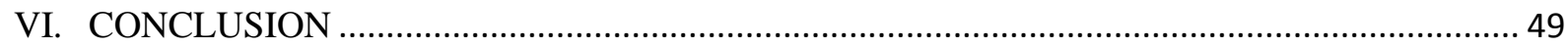

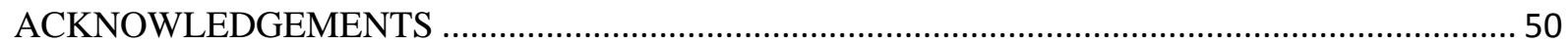

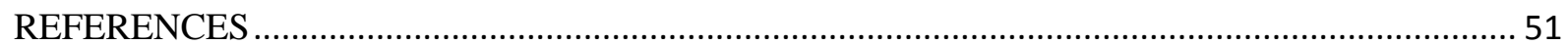




\title{
LIST OF ABBREVIATIONS
}

\author{
8-OHdG 8-hydroxy-2'-dezoxyguanosine \\ aCSF artificial cerebrospinal fluid \\ ASPH asphyxiated experimental group \\ $\mathrm{ASPH}+\mathrm{H} 2$ hydrogen treated asphyxia group \\ $\mathrm{ASPH}+\mathrm{pH} \quad$ asphyxiated experimental group with brain $\mathrm{pH}$ measurements \\ ATP adenosine triphosphate \\ BCAO bilateral carotid artery occlusion \\ CoBF cortical blood flow \\ aEEG amplitude-integrated electroencephalography \\ cEEG conventional electroencephalography \\ EEG electroencephalogram \\ COX-2 cyclooxygenase-2 \\ $\mathrm{CP} \quad$ cerebral palsy \\ CT computer tomography \\ CTR time control experimental group \\ CW cranial window \\ ECG electrocardiograph \\ EPO erythropoietin \\ $\mathrm{FiO}_{2} \quad$ fraction of inspired $\mathrm{O}_{2}$ \\ HI hypoxic/ischemic \\ HIE hypoxic-ischemic encephalopathy \\ HR heart rate \\ $\mathrm{KCl} \quad$ intravenous potassium chloride solution for euthanasia \\ LASCA laser speckle contrast analysis \\ LSCI laser speckle contrast imaging
}


LSCI+BCAO laser speckle contrast imaging group with bilateral carotid artery occlusion

LSCI+ASPH laser speckle contrast imaging group with experimental asphyxia

NAC N-acetylcysteine

NE neonatal encephalopathy

NMR neonatal mortality rate

NOS nitric oxide synthase

NSE neuron-specific enolase

MABP mean arterial blood pressure

MCAO middle cerebral artery occlusion

MDA malondialdehyde

MRI magnetic resonance imaging

MRS magnetic resonance spectroscopy

NMDA N-methyl-D-aspartate

${ }^{\circ} \mathrm{O}_{2} \quad$ superoxide anion

$\mathrm{OH}^{\bullet} \quad$ hydroxyl radical

$\mathrm{ONOO}^{-} \quad$ peroxynitrite radical

$\mathrm{p} \quad$ level of significance

PA perinatal asphyxia

$\mathrm{PCr} \quad$ phosphocreatine

$\mathrm{pH}_{\text {brain }} \quad$ brain interstitial $\mathrm{pH}$

PSM proton selective microelectrode

PTX pneumothorax

RE reference electrode

ROI region of interest

ROS reactive $\mathrm{O}_{2}$ species

RM ANOVA repeated measure of analysis of variance

RR respiratory rate 
SEF secondary energy failure

TGCI transient global cerebral ischemia

TH therapeutic hypothermia

UC umbilical cord

US ultrasonography

XO xanthine oxidase 


\section{LIST OF TABLES AND FIGURES}

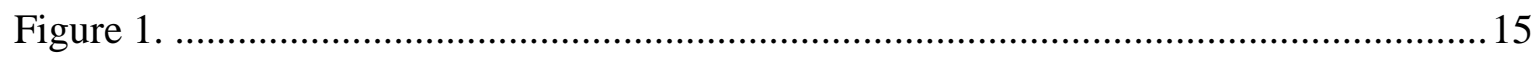

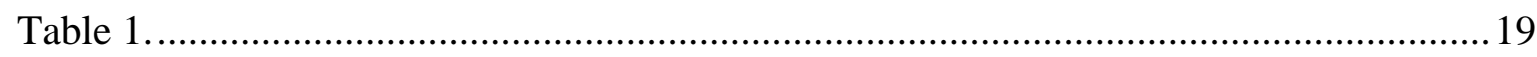

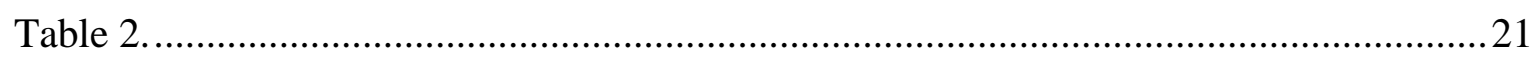

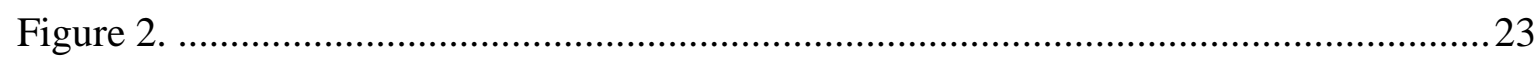

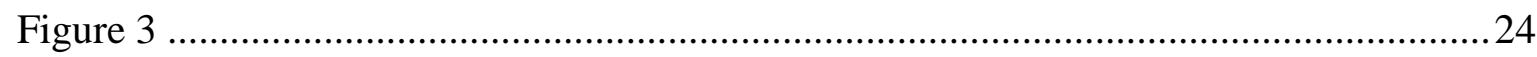

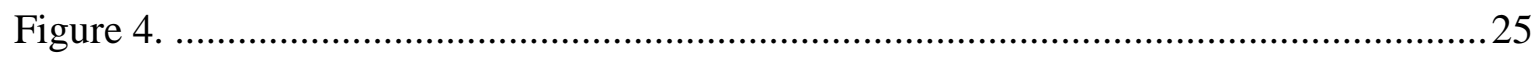

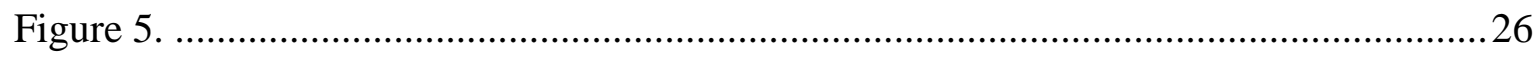

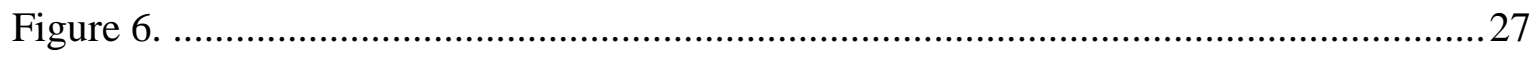

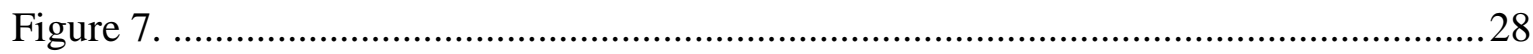

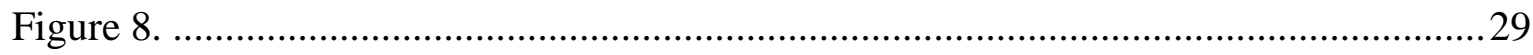

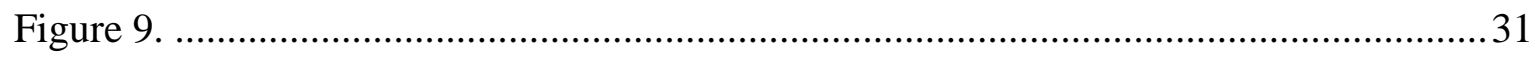

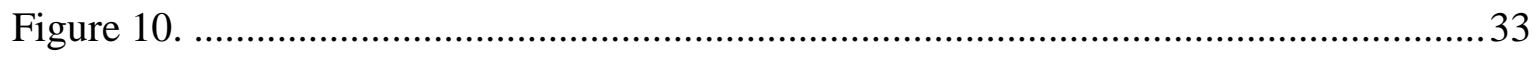

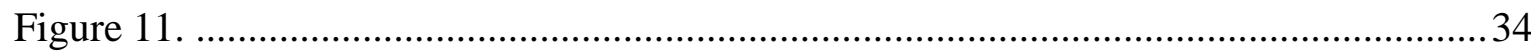




\section{Summary}

Perinatal asphyxia (PA) is one of the leading causes of neonatal mortality and morbidity worldwide. Despite the adequate neonatal resuscitation techniques, hypoxicischemic encephalopathy (HIE) may develop in survivors. Controlled therapeutic hypothermia (TH) became the gold standard care of asphyxiated babies, but its efficacy to prevent long-term disabilities is limited, therefore, additional neuroprotective interventions are required urgently. The newborn pig is regarded as one of the best models for human $\mathrm{PA} / \mathrm{HIE}$ pathology and testing putative therapeutic interventions. The best methodology to elicit pathophysiologically/clinically relevant PA insult in animals is still being debated perhaps resulting in some of the failures of alleged neuroprotective therapies in clinical trials.

Our major aim was to create a translationally relevant newborn piglet PA/HIE model. Accordingly, we investigated the effects of bilateral carotid artery occlusion (BCAO) on cortical blood flow (CoBF), a routinely applied method in the literature to augment hypoxic stress. Then we developed and extensively characterized our new PA/HIE model of PA/HIE and subsequently tested the neuroprotective potential of molecular $\mathrm{H}_{2}$ in our model.

Newborn piglets were anesthetized, instrumented and mechanically ventilated. The effects of BCAO on CoBF were analysed through an implanted cranial window by laser speckle contrast imaging and analysis. PA $\left(6 \% \mathrm{O}_{2}, 20 \% \mathrm{CO}_{2}, 20 \mathrm{~min}\right)$ was induced in separate groups of animals to elicit HIE. One group served for testing the effects of $\mathrm{H}_{2}$ administered during reventilation $\left(2.1 \% \mathrm{H}_{2}, 21 \% \mathrm{O}_{2}, 4 \mathrm{~h}\right)$ on brain electrical activity, neuronal damage and oxidative stress at $24 \mathrm{~h}$ survival).

BCAO did not reduce CoBF. PA elicited clinically relevant severe alterations in cardiovascular and blood gas/metabolic parameters, resulting in depressed electroencephalogram (EEG), extensive neuronal damage associated with oxidative stress assessed by $8-\mathrm{OHdG}$ immunoreactivity. Molecular $\mathrm{H}_{2}$ facilitated EEG recovery, ameliorated neonatal brain injury and alleviated oxidative stress.

In conclusion, our new PA method was able to elicit reproducible, moderate/severe HIE without BCAO. The model was adequate to reveal the neuroprotective effect of molecular $\mathrm{H}_{2}$ administered in a clinically relevant, post-insult time window. The mechanism of neuroprotection appears to involve the reduction of oxidative stress. Encouraged by these promising results, to describe the effect of $\mathrm{H}_{2}$ administration combined with $\mathrm{TH}$ remains our future direction. 


\section{INTRODUCTION}

\section{Epidemiology of child mortality}

Until 1990, 12.7 million children under age 5 died annually, with a child mortality rate of 90 deaths of 1000 live births among them. In addition, neonatal mortality rate (NMR) remained high until the Millennium as approximately 4 million newborns died annually during the neonatal period [1]. In September 2000, the United Nations adopted the Millennium Declaration in New York, including 8 Millennium Development Goals, proclaiming concrete international targets to improve life qualities in areas threatened by hunger and diseases [2]. The $4^{\text {th }}$ task aimed to reduce child mortality worldwide by two-thirds, till 2015. Due to the global efforts, child mortality rate declined by more than half, to 43 deaths of 1000 live births globally [3].

According to a recent global survey (2016) [4], child mortality under age 5 is estimated to be 6.3 million globally [5] whose $44 \%$ ( 2.77 million deaths) occurs during the neonatal period. The first week of life seems to be the most critical period for newborns, as approximately $77 \%$ of the neonatal mortality ( 2.13 million deaths) takes place at that time, particularly on the $1^{\text {st }}$ day of life [4]. It is noteworthy, that NMR shows regional variability: $\sim 0.4 \%$ (out of 1000 live births) in developed countries whilst as high as $3.3 \%$ in low- and middle income (e.g. Africa, Southeast Asia) countries [6]. However, infections (e.g. pneumonia, diarrhoea and malaria) are responsible for $52 \%$ of child mortality under the age of 5, and complications arising from preterm birth along with perinatal asphyxia (PA) ( 1 million and 0.66 million deaths annually) are the leading reasons of neonatal mortality. Finally, according to Hungarian estimations for 2016, NMR decreased first below 4 out of 1000 live births [7].

2. PA and hypoxic-ischemic encephalopathy (HIE)

\subsection{Terms, definitions and symptoms}

Birth asphyxia or PA, is a clinical condition before, during or soon after delivery, characterized by the inability of gas exchange of the fetus or the newborn, resulting in progressive hypoxemia, hypercapnia and mixed acidosis [8]. The respiratory distress induces immediately the centralization of circulation, favoring blood supply to the brain, the heart and the adrenal glands, simultaneously further jeopardising the oxygen $\left(\mathrm{O}_{2}\right)$ and glucose 
availability of the other organs, resulting in hypoxic/ischemic (HI) tissue damage [9]. If cardiovascular compensation fails, the inability to maintain adequate blood flow to the brain further worsens the cerebral metabolic crisis, leading to HIE in survivors. Although the aetiology of PA remains unclear in most cases, many preconceptional (e.g. maternal age or infertility treatment), intrapartum (e.g. maternal diabetes and hypertension, interruption of placental blood flow, uterine rupture, externally compressed umbilical cord [UC] or chorioamnionitis) as well as postpartum risk factors (secondary to cardiovascular or respiratory failure) are thought to increase the incidence of PA [10].

HIE is a subset of a neonatal encephalopathy (NE), perhaps one of the most intensively studied clinical condition in the field of neonatology. As the terms of HIE and NE are often used imprecisely in the literature, the American College of Obstetricians and Gynaecologists along with the American Academy of Paediatrics defined the following criteria for the diagnosis of HIE, related to PA: 1) severe acidosis in umbilical arterial blood $(\mathrm{pH}<7.0$ and base deficit $>12 \mathrm{mmol} / \mathrm{l}), 2)$ the persistence of low (0-3) Apgar scores [11], 3) neonatal neurologic disorders (e.g. coma, seizure activity or muscle hypotonia) and 4) multi organ failure [12]. Thus, if these criteria are met at birth, it is likely that PA can be responsible for perinatal brain damage, causing HIE. NE describes a broader clinical presentation of impaired neurological functions with the most notable symptoms of neurologic depression, including low level of consciousness or coma, seizures, muscle tone and reflex abnormalities along with respiratory problems and impaired feeding during the neonatal period [13]. However, these symptoms are not specific for PA aetiology, in addition, sometimes it is difficult to distinguish PA-induced HIE from other causes of NE during the first minutes of life [14]. Consequently, NE is not necessarily caused by PA but can be the consequence of other disorders as well as neonatal encephalitis, intracranial hemorrhage, birth trauma, neurodevelopmental or metabolic disorders, such as hypoglycaemia, hyperammonaemia, hyperbilirubinaemia, mitochondrial disorders and errors of amino acid metabolism [15]. Importantly, Volpe advised against the use of the term "NE" when clinical and neuroimaging characteristics of neonatal brain damage are related to HI events [13]

The outcome of PA shows great variability as infants can recover 1) completely, without neurological impairments, 2) with mild consequences (e.g. with attention deficit disorder), 3) or in more severe cases HIE can cause death or permanent symptoms of NE, leading subsequently to long-term neurodevelopmental sequelae including seizures, mental and somatic retardation as well as cerebral palsy $(\mathrm{CP})$. CP is the commonest motor disorder in 
childhood, however, the etiology of $\mathrm{CP}$ is still unknown in most cases. Its incidence is estimated to be 2 out of 1000 live births [16]. CP is defined as a life-long, non-progressive motor disability and classified into 2 forms according to whether pyramidal (the spastic type with muscle hypertonia and increased deep-tendon reflexes) or extrapyramidal lesions (the dyskinetic type with choreoathetosis, coordination problems and postural control deficits) are manifested [17], imposing a great financial burden on the families, the health care providers and the society as well [18]. Consequently, preventing the development of CP during early life is one of the greatest challenges of neonatology.

In summary and for clarity, HIE is defined as a type of NE with evidence of PA. PA can eventually cause life-long neuronal impairments in survivors, called $\mathrm{CP}$, through the progress of NE [19].

\subsection{Clinical manifestations and tools for grading HIE}

Following resuscitation and stabilization of the newborn, clinical management is initiated soon in the neonatal intensive care unit. It is noteworthy that beside the neurological dysfunctions arising from HIE, systemic complications of PA also contribute collectively to neonatal brain damage. These HI complications may include the following disorders: acute renal tubular and hepatic necrosis, necrotizing enterocolitis, adrenal insufficiency and cardiomyopathy [20]. Neurological examination, laboratory tests along with neuroimaging and electrophysiology techniques altogether help clinicians to identify the degree of perinatal cerebral injury and to grade the severity of HIE, in addition, to predict late neurodevelopmental outcome.

The clinical status of the neonate in the $1^{\text {st }}$ and the $5^{\text {th }}$ minute of age is assessed by a standardized, well-known scoring system: the Apgar scores, including the description of the color, heart rate, muscle tone, reflexes and respiration of the newborn, introduced by Virginia Apgar in 1952 [11]. However, Apgar scores are not specific for PA aetiology. In 1976, Sarnat and Sarnat proposed a scoring system [21] providing an accurate scale to determine the degree of perinatal brain damage. The scale classifies newborns into three stages of HIE as mild (stage I), moderate (stage II) and severe (stage III), based on the combined neurologic (level of consciousness, muscle tone, tendon reflexes, seizures, complex neonatal reflexes and autonomic functions) and electroencephalography (EEG) findings. Although these scoring systems are widely accepted and guide also clinicians in neonatal resuscitation and 
postasphyxial care, but unfortunately, their accuracy to predict late neurodevelopmental outcome is low $[22,23]$.

On the other hand, the analysis of UC $\mathrm{pH}$ and blood gases along with base deficit is fundamental to meet the criteria of PA, the severity of HIE cannot be graded according to these laboratory values alone [23], thus, more sensitive and complementary methods were required.

Neuroimaging with cranial ultrasonography (US), computer tomography (CT) and Magnetic Resonance Imaging (MRI) are routinely used for early visualization of perinatal brain damage with their merits and limitations. Cranial US provides easy and fast access to the lateral ventricles, moreover, the ability to monitor cerebral hemodynamics. Early US was reported to determine accurately the presence and the extent of cerebral edema soon after PA, however, it has limitations in detecting intracranial bleedings [24]. Interestingly, a recent meta-analysis revealed the lower accuracy of CT alone against MRI in assessing brain damage, as CT delineated poorly the classic patterns of HI brain damage, including deep grey matter, white matter and brainstem injury [25]. The use of MRI with Magnetic Resonance Spectroscopy (MRS) has increased dramatically and became the principal non-invasive tool to assess the severity of HIE over the past few decades, especially in the United States. This technique offers the chance to visualize both acute and chronic perinatal brain lesions during the antepartum and postpartum periods, moreover, to analyse real time the level of cellular metabolites in vivo. [26]. MRI along with MRS help clinicians to select safely and early critically sick neonates who could profit from neuroprotective interventions [27]. In addition, obtaining a time-series of MRI/MRS data offers the possibility to evaluate the effectiveness of putative neuroprotectants on the neonatal brain, to predict faithfully the outcome of cerebral injury (even at 4 years of age [28]), finally, to consider the withdrawal of therapy in non-responding patients. The extent of neonatal brain injury is influenced by many factors such as the duration and severity of PA. In the past, extensive amounts of autopsies along with the recent help provided by MRI studies helped to describe the classic patterns of HI brain injury in term asphyxiated neonates. The most common findings include: 1) diffuse neuronal necrosis or necrosis located to the cortex with the deep grey matter or to the deep grey matter with the brainstem, 2) parasagittal injury along the watershed areas and less often 3) periventricular leukomalacia or 4) focal ischemic necrosis [29]. Despite the numerous advantages of MRI and MRS, neuroimaging is still expensive and MRI compatible tools are also required. 
Conventional electroencephalography (cEEG) recording is a cheap, safe and noninvasive method to visualize real-time cerebrocortical functional activity. Although performing the measurements is not so complicated but the interpretation of the EEG patterns requires special training. As neonatal EEG activity develops with age, moreover, the interpretation and recognition of pathophysiologic EEG findings is difficult, numerous classification protocols were proposed by clinicians for the assessment of cerebral functions [30]. According to these recommendations, EEG recordings are scored gradually according to the presence or absence of the hemispheric symmetry, the sleep-wake cycles, the frequency, the continuity and the amplitude of background electric activity. However, there is still no consensus how to grade the severity of HIE in term asphyxiated newborns. On the other hand, EEG recordings can quickly identify babies for therapeutic interventions with signs of HIE in the acute phase soon after birth and can also be a good prognostic tool to predict late neurodevelopmental outcome. Normal and moderately abnormal EEG patterns recorded as early as 6 hours of life in asphyxiated babies have been reported to be good predictors of neurologic outcome at 2 years [31].

From cEEG recordings, amplitude-integrated EEG (aEEG) signal can be converted [32]. During the past few years, aEEG spread widely in neonatal intensive care [33] as it is a simplified, time-compressed EEG signal on a semi-logarithm scale. Thus, the easier interpretation of aEEG patterns in term neonates favors for its use, although the simultaneously recorded raw EEG signal can improve its sensitivity and specificity in determining the prognosis of critically ill neonates.

\section{The pathophysiology of neonatal brain damage}

\subsection{Primary energy failure}

The molecular mechanisms of HI-induced brain damage arises principally from animal experiments [34-36]. Briefly, hypoxemia with reduced glucose delivery to the brain are thought to be the initial steps leading to the activation of biochemical cascades in neurons and glial cells. First, hypoxemia shifts aerobic metabolism to anaerobic glycolysis, causing prominent tissue acidosis. The subsequent depletion of high energy compounds (adenosine triphosphate $[\mathrm{ATP}]$ and phosphocreatine $[\mathrm{PCr}]$ ) results in the inability to maintain adequate function of the sodium-potassium pumps and consequently of the sodium-calcium exchangers. Therefore, intracellular calcium $\left(\mathrm{Ca}^{++}\right)$, sodium along with cell volume homeostasis break, inducing depolarization, swelling (cytotoxic edema) or lysis [37], and the 
activation of $\mathrm{Ca}^{++}$dependent downstream neurotoxic cascades in the cells. High intracellular $\mathrm{Ca}^{++}$contributes on the one part to the activation of various proteases, lipases, endonucleases and the nitric oxide synthase (NOS) enzyme, on the other hand, via depolarization, $\mathrm{Ca}^{++}$entry initiates the release of excitatory neurotransmitters, glutamate and aspartate into the extracellular space [38]. Glutamate floods the synaptic cleft and in turn, these chains of molecular events will culminate in glutamate receptor overactivation-related cytoplasmic $\mathrm{Ca}^{++}$ accumulation, neuronal depolarisation as well as endoplasmic reticulum and mitochondrial dysfunctions [39]. Finally, such biochemical responses can eventually lead to death.

Fundamentally, two types of neuronal cell death were characterized: necrosis is principally described after severe HI events as an early type of death, whereas less severe insults can result in delayed, highly coordinated, energy consuming "suicide programme", called apoptosis [40]. Interestingly, a recently rediscovered molecular mechanism, called autophagy, is under extensive research these years [41]. Autophagy, is a reversible, transitory period in a cell, promoting cell survival via degrading and recycling proteins and eliminating the damaged organelles, even after PA [42, 43]. However, in case of unfavorable conditions or irreversible cell damage the outcome of the autophagic machinery can also result in cell death. All of these mechanisms in the brain coexist after PA.

\subsection{Reperfusion and secondary energy failure (SEF)}

Successful resuscitation ameliorates arterial blood gases and cerebral perfusion eliminating cerebral hypoxia/ischemia. The restored fuel supply to the brain at least partially decreases extracellular glutamate levels limiting excitotoxicity and reduces cell swelling. On the other hand, this early reoxygenation/reperfusion period after HI is characterized by oxidative stress as xanthine oxidase (XO), cyclooxygenase-2 (COX-2) and NOS enzymes were reported to be upregulated in the brain, producing predominantly reactive $\mathrm{O}_{2}$ species (ROS, e.g. hydroxyl radical $\left[\mathrm{OH}^{*}\right]$ and peroxynitrite radical $\left.\left[\mathrm{ONOO}^{-}\right]\right)$. In addition, mitochondrial cytochrome $\mathrm{C}$ oxidase is also overactivated by the $\mathrm{Ca}^{++}$cytotoxicity leading also to the extensive production of ROS. Lipid membranes, proteins and the DNA are the major targets of such oxidative storm, resulting in lipid- and protein peroxidation with dysfunctions along with the irreversible damage to the DNA [44]. Beside, toxic radicals deplete the endogenous antioxidant systems (e.g. glutathione) as well and further trigger mitochondrial damage, causing the activation of proteolytic caspase proteins. Reperfusion injury can also induce early, proinflammatory gene expression (e.g. adhesion proteins, cytokines, chemokines and prostaglandins [45]) leading to the activation and accumulation of 
microglia and neutrophils in the injured areas, promoting the opening of the blood-brain barrier [46] and further aggravating neuronal damage [47].

MRS studies led first to the concept of SEF [48] in infants with HIE. Notably, the recovery from cerebral hypoxia/ischemia can result in the complete restoration of high energy metabolite levels (PCr), measured by MRS in survivors of PA. Although the primary energy failure was abolished for several hours (called as the "latent phase") in these newborns, however, despite the adequate resuscitation techniques and postasphyxial intensive care [49], a SEF was observed soon. This secondary deterioration of cerebral oxidative metabolism was demonstrated as early as 6-24 hours and the metabolic crisis peaked approximately between 24-48 hours after resuscitation. It is speculated that progressive damage to the mitochondria disrupts the process of oxidative phosphorylation, eliciting a "delayed injury" or SEF in neurons [50]. Thus, the existence of a window preceding the SEF in severely asphyxiated neonates may offer a chance to introduce putative neuroprotective interventions to delay and/or mitigate the second drop in ATP/PCr levels, promoting cell survival [10].

\subsection{Tertiary phase}

Inflammatory and reparative processes in the brain after PA may extend for weeks or even years in children, surviving HIE [51]. The tertiary brain injury represents the period of gliosis, epigenetic changes, persistent activation of inflammatory cells along with tissue reparation. Interestingly, at cellular level, persisting intracellular lactic alkalosis was detected after a year in children surviving PA, with adverse neurologic outcome [52].

\subsection{Metabolic biomarkers of brain injury}

Although clinical examination, neuroimaging and electrophysiology tests are the primary diagnostic tools of HI-induced neonatal brain injury, routine measurement of brain constituents in body fluids may also provide an indirect, quantitative diagnostic tool about the extent of brain injury [53]. Numerous cerebral biomarkers (e.g. S100B protein, activin A, adrenomedullin, glial fibrillary acidic protein) along with inflammatory cytokines (e.g. IL-1, IL-6, IL-8, tumor necrosis factor alpha) [54] were serially measured in newborns surviving PA, however, there is still no early and specific serum indicator of cerebral injury at birth offering good prognostic value for late neurodevelopmental outcome. Interestingly, neuronspecific enolase (NSE) detected after PA in either cerebrospinal fluid [55] or blood [56] during the $1^{\text {st }}$ day of life was found to be an early and promising biomarker of neonatal brain damage as protein levels in asphyxiated babies correlated well with the clinical manifestation 
of HIE. Furthermore, plasma NSE levels during the $2^{\text {nd }}$ and $3^{\text {rd }}$ day of life after PA showed also high predictive value for late neurological outcome [57].

\section{Animal models of PA/HIE}

In biomedical research, animal models offer an invaluable aid to reveal unknown mechanisms of pathophysiological processes, moreover, they open the door to study the pathophysiology of PA/HIE and to test putative neuroprotective therapies. The earliest published attempts describing the effects of asphyxiation on animals were probably performed as early as two centuries ago. From a historical perspective it is very interesting that these $19^{\text {th }}$ century observations reported on the effects of submerging rabbits and rats under water, thus establishing the first steps towards discovering the physiologic responses of asphyxiation [58, 59]. Subsequently, pregnant rabbits $[60,61]$ as well as pregnant guinea pigs [62] were introduced as affordable models of PA where fetal asphyxiation was performed via UC occlusion. From the 1950s, new large animal models were created closing the translational gap between human neonates and animal models. In order to study the effects of the interruption of cord blood flow, even fetal Rhesus monkeys were exposed to placenta detachment [63]. Interestingly, one year later, Levine established an anoxic-ischemic encephalopathy adult rat model based on unilateral carotid artery occlusion with hypoxic ventilation [64] that was adjusted for 7-day-old rat pups by Rice and Vannucci. This model has since become the gold standard method to elicit PA/HIE in newborn rats [65].

The choice of the animal species and the method to induce asphyxia is critical to reproduce the metabolic (e.g. plasma glucose and lactate, brain excitatory amino acids levels, blood gas values, cerebral oxygenation values) and functional (e.g. core body temperature, heart rate, blood pressure, central venous pressure, respiratory changes, EEG activity) changes observed in the human neonate. However, the exact cause, onset, duration as well as the severity of PA have not been fully discovered yet, resulting in the introduction of a great number of further animal models, all with their respective merits and limitations. Small animal (e.g. guinea pig, rat, rabbit, mouse) models contributed significantly to exploring the molecular mechanisms in the brain as costs and availability favor their application. In addition, easy handling and husbandry provide long-term survival but the interpretation of results for clinical relevancy must be done carefully. On the other hand, body size of large gyrencephalic animals (e.g. sheep, lamb, goat, dog, rhesus monkey and pig) allows multiple instrumentation and their brain development extends around birth as in the human neonate 
[66], however, their prohibitive costs, seasonal availability and ethical concerns can limit their use in research. Above these animal models, the newborn pig became the deputy of the term neonate.

5. The newborn pig as model of the neonate

Pig in biomedical research was already employed in the 1960s and the species was proposed as an adequate candidate of the human model because of similar genetic and physiologic characteristics $[67,68]$. Although gestation of the sow lasts approximately only for 4 months, the developmental stages of the animal still show similarities to the human intrauterine development [69], therefore, prenatal pig has become a valuable model to investigate human embryology and teratology as well. The gyrification pattern and the brain developmental spurt at birth [70] share similarities with the term human neonatal brain around delivery $[71,72]$. However, there are notable differences in brain maturation as pigs are able to walk within hours after birth and can be weaned from their sows early. The swine and the human brain mass at birth represent a very similar $25 \%$ and $27 \%$ of the final adult brain, respectively [66]. Dobbing and Dickerson determined that the brain mass growth in the infant during the first 12 months of life corresponded to brain development of the pig during the first 12 weeks $[70,73]$, further increasing the significance of pigs in paediatric research as well.

It is noteworthy, that natural PA occurs in the fetal pig around birth. Due to the multiparity, the effects of uterine contractions during delivery increase the length of HI events and the risk for placental detachment of unborn animals [74]. Randall's extensive research on swine delivery revealed that $20-25 \%$ of pig perinatal mortality is due to UC rupture-related intrauterine stillbirth [75-78]. Thus, birth order has been suggested to have an impact on cord $\mathrm{pH}$ and $\mathrm{pCO}_{2}$ levels [79]. These metabolic changes are similar to values reported from human asphyxiated neonates thus closing the translational gap between pigs and neonates [80].

During the past few decades numerous PA/HIE swine models were introduced ranging from $\mathrm{HI}$ insults restricted to the brain (e.g. cerebral ischemia induced by raised intracranial pressure [81] or vessel ligation [82]) to global asphyxiations (e.g. bilaterally induced pneumothorax [PTX] [83] or the cardiac arrest model [84]). Nowadays, hypoxic ventilation with [85] or without [86] bilateral carotid artery occlusion (BCAO) has become the gold standard way to induce PA, however, hypercapnia, also a major hallmark of PA, is not always developed at this type of insult. Moreover, the clinical relevance of mechanical vessel occlusion (BCAO) is questionable. Thus, an ideal model should display all metabolic 
hallmarks of PA and should be free of mechanical vessel occlusion, in addition, it should produce reproducible and clinically relevant HI brain damage.

\section{Neuroprotective strategies}

Before the introduction of therapeutic hypothermia $(\mathrm{TH})$, the management of infants with signs of HIE consisted only supportive care along with anticonvulsive therapy. Then TH became the proven, gold standard care of asphyxiated babies [87]. However, TH cannot offer full neuroprotection, and currently no other clinically established interventions were reported to complement its neuroprotective effect. In addition, not all of the survivors of PA may profit from $\mathrm{TH}$, on the other hand, numerous side effects of $\mathrm{TH}$ were also reported related to total body cooling (e.g. bradycardia, hypotension, hemoconcentration, hyperkalemia during rewarming, thrombocyte activation and aggregation and so on [88]). Thus, complementary, safe and aimed neuroprotective treatments of HIE are still urgently required.

Interestingly, numerous substances showed neuroprotection in various small and large animal PA/HIE models, however, not all of them (e.g. $\mathrm{Ca}^{++}$channel blocker: nicardipine; magnesium) could pass the clinical trials, due to probably the lack of neuroprotection or the observed side effects. On the other hand, new promising candidates are currently under active scope of research [89]. For the sake of brevity, xenon inhalation, not alone but combined with TH was proved to be neuroprotective in a newborn piglet model of PA/HIE [90]. In clinical trials, cardiovascular stability was preserved in asphyxiated babies during xenon administration, however, the need for a closed-circuit delivery along with a recycling system and its expensive cost, limit the routine application of the gas in neonatal intensive care [91]. Allopurinol administration, an inhibitor of $\mathrm{XO}$, was effective in reducing oxidative stress in numerous PA/HIE animal experiments, although only few clinical trials could report beneficial effects of the drug especially only in moderately asphyxiated babies [92]. Interestingly, a recent meta-analysis (2008) of 3 clinical trials did not reveal significant benefit from allopurinol administration [93]. N-acetylcysteine (NAC), the antidote of paracetamol intoxication, has excellent antioxidant and free radical scavenging properties and afforded marked neuroprotection in numerous $\mathrm{HI}$ and intrauterine inflammation rat and piglet models, with or without the combination of TH [94]. Although NAC penetrates the placenta easily, but due to its ample side effects [95] the clinical use of NAC has not spread yet in perinatal medicine. Currently, the most attractive candidates for future clinical trials are perhaps melatonin and erythropoietin (EPO). Therapeutic application of these substances in 
neonatal asphyxia animal models is appealing because of the safety of their administration [96]. Melatonin was found to induce neuroprotection via its antioxidant, anti-inflammatory and anti-apoptotic effects observed in preclinical studies [97]. Furthermore, EPO offers antiinflammatory capacity and recent clinical trials have already found improved outcome in asphyxiated babies treated with EPO [98]. Phenobarbital, one of a widely used anticonvulsants in neonates could show neuroprotection after PA and augmented hypothermic neuroprotection as well in term neonates [99]. After all, an ideal neuroprotectant should fulfill the following criteria: 1. promising results obtained in reliable translational animal models, 2 . easy and cheap administration at bedside, 3. no/minimal side effects, 4. ability to pass the biological membranes and the barriers, and finally 5 . good clearance.

In 2007, Ohsawa et al. introduced molecular hydrogen $\left(\mathrm{H}_{2}\right)$ as a putative antioxidant gas selectively reducing the levels of $\mathrm{OH}^{-}$and $\mathrm{ONOO}^{-}$radicals in primary culture of neocortical cells and cell free systems. Moreover, they demonstrated that $\mathrm{H}_{2}$ inhalation dosedependently decreased the infarct volume at 1 day in focal ischemia adult rat model. The most effective concentration for neuroprotection was found between $2-4 \%$, in addition, $\mathrm{H}_{2}$ suppressed the progression of brain damage at 1 week survival as well [100]. Since then, ample preclinical and clinical studies in almost every field of science reported the beneficial effects of this molecule regarded as an inert gas previously [101]. Supposedly, $\mathrm{H}_{2}$ can effectively reduce the amount of $\mathrm{OH}^{*}$ and $\mathrm{ONOO}^{-}$radicals, however, the pharmacological targets of the gas may not be limited to this mechanism, and many details of the actions of $\mathrm{H}_{2}$ are not discovered yet. It is also noteworthy that beside its antioxidant effect, antiinflammatory, cytoprotective and cell signal modulatory effects of $\mathrm{H}_{2}$ were also reported [102].

$\mathrm{H}_{2}$ administration afforded neuroprotection in numerous cerebral $\mathrm{HI}$ animal models; for instance, Li et al. observed reduced oxidative damage and cytokine production along with increased activities of antioxidant enzymes after focal cerebral ischemia in adult rats [103] or Cai et al. found that $\mathrm{H}_{2}$ treatment significantly decreased also oxidative stress, suppressed caspase- 3 activity and inhibited microglia activation in neonatal rat PA/HIE model [104]. To the best of our knowledge, our laboratory was the first employing $\mathrm{H}_{2}$ gas in large animal PA/HIE model [105], however, the beneficial effects of the neuroprotectant was found to be limited as the employed experimental asphyxia (induced by clamped endotracheal tube and halted ventilation) elicited only moderate $\mathrm{HI}$ brain injury in piglets evaluated by neuropathology at 24 hours of survival [105]. Consequently, the efficacy of inhaled $\mathrm{H}_{2}$ to 
afford significant neuroprotection in a more severe PA/HIE piglet has been thus unanswered yet. 


\section{AIMS}

During the past 50 years, a large number of newborn piglet models were proposed to study the pathophysiology of PA/HIE with various clinical relevancy. The limitations were due to either the application of mechanical vessel occlusion or the lack of metabolic hallmarks (e.g. hypercapnia) of PA at the end of the experimental insults that strongly influence neuronal damage and survival. TH offers neuroprotection in asphyxiated babies but complementary interventions are required to augment the beneficial effects of cooling. Although $\mathrm{H}_{2}$ afforded neuroprotection in various HI animal models but was not tested in a severe, large animal PA/HIE model yet. Moreover, beside the activation of asphyxia-induced intracellular cascade mechanisms, brain interstitial $\mathrm{pH}\left(\mathrm{pH}_{\text {brain }}\right)$ plays also a crucial role in neuronal survival and excitability, however, in vivo recordings of $\mathrm{pH}_{\text {brain }}$ in the literature are scarce. To address these issues, the aims of the present work were the followings:

1.) We investigated the effects of BCAO on cerebrocortical microcirculation in newborn piglets both under normoxic and hypoxic conditions to study the necessity and efficacy of mechanical vessel occlusion on the severity of $\mathrm{HI}$ insult.

2.) We established and characterized profoundly a new PA/HIE piglet model with 24hour survival. We collected unique data on the $\mathrm{pH}_{\text {brain }}$ changes during asphyxia and the subacute phase of HIE.

3.) Using our new PA/HIE model we sought to test the potential neuroprotective effects of molecular $\mathrm{H}_{2}$. We administered $\mathrm{H}_{2}$ after PA to enhance the translational potential of the study

4.) Finally, we assessed the effect of $\mathrm{H}_{2}$ on cortical oxidative neuronal damage to test whether $\mathrm{H}_{2}$ effects are in accordance with its proposed antioxidant mechanism of action. 


\section{MATERIALS AND METHODS}

1. Animals

Newborn ( 1-day-old) male Large-White piglets (body weight: $1.5-2.5 \mathrm{~kg}, \mathrm{n}=48$ ) were obtained from a local company (Pigmark Ltd., Co., Szeged, Hungary) and delivered to the laboratory on the morning of the experiments. All procedures were approved by the Animal Care and Use Committee of the University of Szeged. Anesthesia was induced by intraperitoneal injection of sodium thiopental (45 mg/kg; Sandoz, Kundl, Austria). Piglets were intubated via tracheotomy and artificially ventilated with warmed, humidified room air $\left(21 \% \mathrm{O}_{2}\right.$, balance $\mathrm{N}_{2}$ ) that could be supplemented with $\mathrm{O}_{2}$ via a pressure-controlled neonatal ventilator. Respiratory settings (fraction of inspired $\mathrm{O}_{2}\left[\mathrm{FiO} \mathrm{O}_{2}\right]$ : 0.21-0.25; respiratory rate [RR]: 30-35 1/min, peak inspiratory pressure: $120-135 \mathrm{mmH}_{2} \mathrm{O}$ ) were adjusted to maintain blood gas values and $\mathrm{O}_{2}$ saturation in the physiologic range. The right femoral vein was catheterized under aseptic conditions to maintain anesthesia/analgesia with a bolus injection of morphine (100 $\mu \mathrm{g} / \mathrm{kg}$; Teva, Petach Tikva, Israel) and midazolam (250 $\mu \mathrm{g} / \mathrm{kg}$; Torrex Pharma, Vienna, Austria), then with continuous infusion of morphine $(10 \mu \mathrm{g} / \mathrm{kg} / \mathrm{h})$, midazolam $(250 \mu \mathrm{g} / \mathrm{kg} / \mathrm{h})$ and fluids (5\% glucose, $0.45 \% \mathrm{NaCl} 3-5 \mathrm{ml} / \mathrm{kg} / \mathrm{h})$ throughout the whole experiment, as artificial ventilation-induced stress [106] affects the neuro-endocrine system in intubated neonates. A second catheter was placed either into the right femoral artery in piglets, subjected to $\mathrm{BCAO}$, or into the right carotid artery in piglets, subjected to $\mathrm{PA} / \mathrm{HIE}$ research, for continuous monitoring of mean arterial blood pressure (MABP) and heart rate (HR). In the latter case, the carotid artery was chosen as ligation of the femoral artery resulted in critical ischemia of the hindlimb over the chosen survival period (personal observations), in contrast, unilateral carotid artery occlusion has been shown to unaffect cerebral blood flow [107]. To elicit BCAO, remotely controlled vascular occlusion cuffs (OC A2, In Vivo Metric, Healdsburg, CA, USA) were secured around both exposed common carotid arteries. After suturing the surgical incisions, animals were divided into 6 groups (Fig.1):

1) group $\mathrm{LSCI}+\mathrm{BCAO}(\mathrm{n}=7)$ for evaluating the effects of $\mathrm{BCAO}$ on cerebrocortical microcirculation under various ventilation conditions;

2) in group CTR $(n=7)$ time control animals without asphyxic stress survived 24 hour;

3) in groups ASPH (n=8) animals were asphyxiated and reventilated with room air for 24 hours; 
4) $\mathrm{ASPH}+\mathrm{H}_{2}(\mathrm{n}=8)$ animals were asphyxiated and reventilated with molecular $\mathrm{H}_{2}$ for the initial 4 hours of survival, than with room air for the remaining time;

5) group $\mathrm{ASPH}+\mathrm{pH}(\mathrm{n}=13)$, for measuring $\mathrm{pH}_{\text {brain }}$ changes;

6) and finally, group LSCI+ASPH $(n=5)$ served for evaluating the effects of experimental asphyxia on cerebrocortical microcirculation and finally.

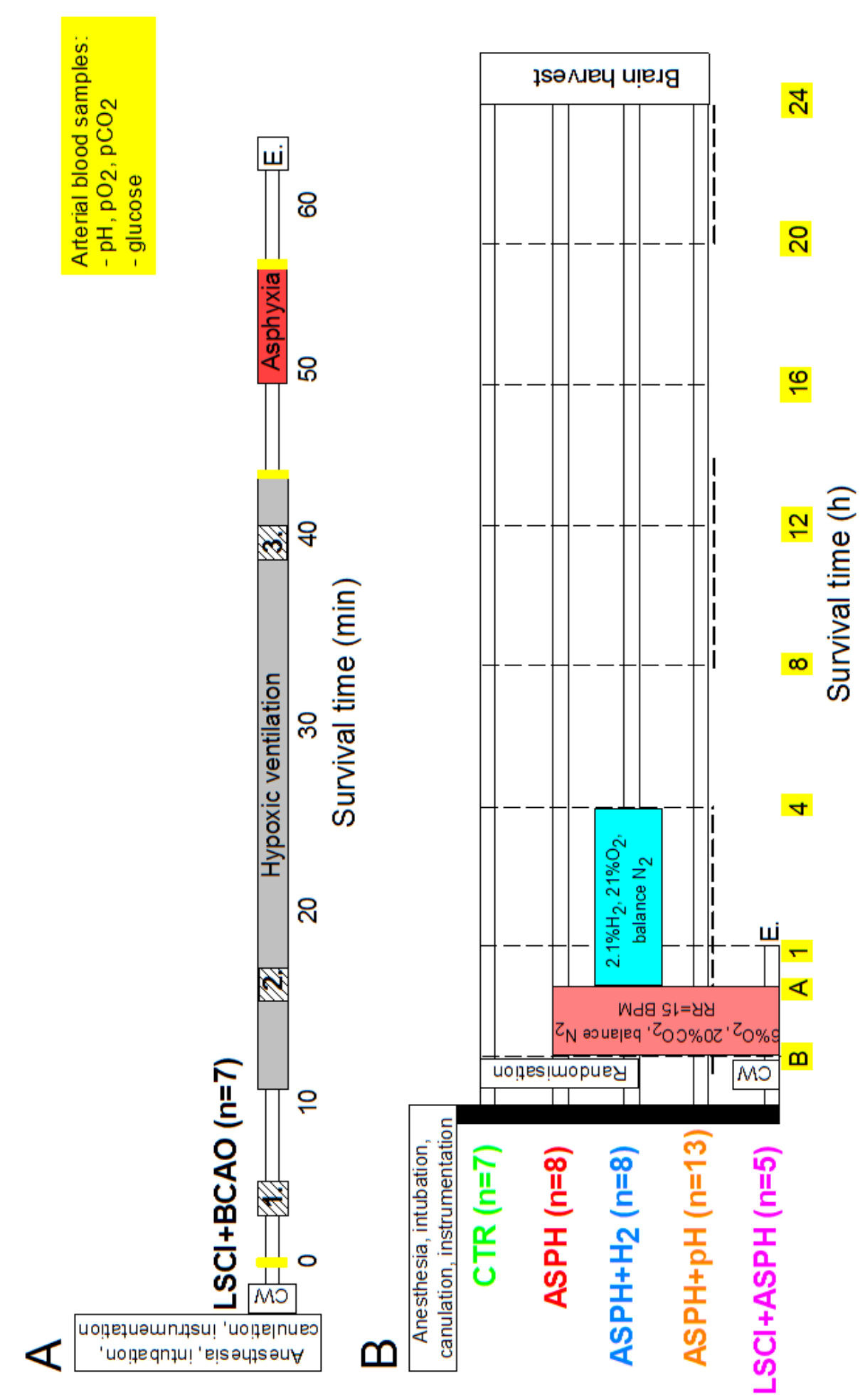


Figure 1. Experimental protocols and groups. Panel A: first, to test the effects of mechanical vessel occlusion with LSCI/LASCA on cerebrocortical microcirculation, animals of group LSCI+BCAO $(n=7)$ were equipped with a closed cranial window $(\mathrm{CW})$. Transient $\mathrm{BCAO}$ was performed 3 times in mechanically ventilated piglets, as indicated by the hatched squares: the $1^{\text {st }}$ one under baseline conditions (room air ventilation), meanwhile the $2^{\text {nd }}$ and $3^{\text {rd }}$ ones under 35 min $10 \%$ hypoxic $\left(10 \% \mathrm{O}_{2}\right.$, balance $\mathrm{N}_{2}$, grey) ventilation. Then, animals were reoxygenated with room air for 5 min and ventilation was halted to induce asphyxia (dark red) for $7 \mathrm{~min}$ followed by $5 \mathrm{~min}$ room air reventilation. The biological zero was recorded after the euthanasia (E). Panel B: Second, the potential neuroprotective effects of molecular $\mathrm{H}_{2}$ against asphyxia-induced HIE were tested in 3 groups. After randomization, animals of group CTR ( $n=7$, green) served for normoxic, time control animals, and were mechanically ventilated with room air during the observation period. Piglets in groups ASPH ( $\mathrm{n}=8$, red) and $\mathrm{ASPH}+\mathrm{H}_{2}\left(\mathrm{n}=8\right.$, blue) were subjected to 20 min hypoxic-hypercapnic $\left(6 \% \mathrm{O}_{2}, 20 \% \mathrm{CO}_{2}\right.$, reduced RR, stopped glucose infusion, light red) insult. Animals of group ASPH were reventilated with room air during the 24-hour survival period, whereas animals in group $\mathrm{ASPH}+\mathrm{H}_{2}$ were switched to a gas mixture, containing $2.1 \% \mathrm{H}_{2}(+21 \%$ $\mathrm{O}_{2}$, balance $\mathrm{N}_{2}$, cyan) for 4 hours, then to room air for the remaining time of the experiment. In a separate group of piglets (group $\mathrm{ASPH}+\mathrm{pH}, \mathrm{n}=13$, orange), $\mathrm{pH}_{\text {brain }}$ measurements were performed at given time intervals, indicated by the scattered lines. Then, brains at 24 hour of survival were harvested and processed for histopathology analysis. Finally, to characterize CoBF velocity changes in our currently presented PA piglet model with the LSCI/LASCA technique, animals in group LSCI+ASPH ( $\mathrm{n}=5$, purple) were equipped with closed CW and experimental asphyxia was also performed. Animals survived a brief $(\sim 1 \mathrm{~h})$ observation period afterwards. Arterial blood samples were checked at given timepoints (yellow). Nevertheless, physiologic parameters of the piglets (core temperature, $\mathrm{O}_{2}$ saturation, HR, MABP, EEG and ECG) were recorded continuously.

\section{Laser speckle contrast imaging (LSCI) and analysis (LASCA)}

Animals of groups LSCI+BCAO and LSCI+ASPH served for cerebrocortical microcirculation measurements.

Initially, animals were placed in prone position with the head fixed in a stereotactic frame. Then, the scalp was retracted and following a circular craniotomy and removal of the dura mater, a closed cranial window (CW) with 3 injectible ports was inserted over the left parietal region [108]. The CW was sealed with bone wax, cyanoacrylate and dental acrylate (Lang Dental Manufacturing Co Inc, Wheeling, IL, USA). After 45 min incubation, the subarachnoidal space under the $\mathrm{CW}(\sim 3 \mathrm{ml}$ chamber volume) was filled with warmed, $\mathrm{pH}$ equilibrated artificial cerebrospinal fluid (aCSF) through the ports. Our custom-designed speckle imager and the LASCA software allow to monitor rapid changes in tissue perfusion by determining the autocorrelation decay time $(\tau)$ of interference patterns produced by laser light (laser speckle) scattered from moving particles (red blood cells). The average velocity is directly proportional to $1 / \tau$. Accordingly, the $\mathrm{CW}$ was illuminated with a polarized light of near-infrared diode laser ( $\lambda=808 \mathrm{~nm}, 200 \mathrm{~mW}$, DL-8141-002 Sanyo Electric Co., Japan) and speckle images were recorded $(1 \mathrm{~Hz}, 2 \mathrm{msec})$ through an operating microscope by a PLB741F monochrome camera (PixeLINK, Ottawa, Canada), visualised online with a custommade software in LabVIEW (National Instruments Co., Austin, Texas, USA) and stored on a personal computer. LASCA was performed offline; regions of interests (ROI, 4 for each LSI 
image in each animal) were selected over the cortical parenchyma not obstructed by surface pial vessels or over pial arteries using a ROI size of $5 \times 5$ pixels $\left(\sim 100 \mu \mathrm{m}^{2}\right)$. The determined $1 / \tau$ values were normalized for baseline, and the data were then expressed as relative changes from baseline. Pial arteriolar diameter changes were determined offline from laser speckle contrast images using our custom-designed software and were expressed as relative changes from baseline.

The experimental protocols were the following: in group LSCI+BCAO $(n=7)$, after obtaining 2 min baseline, transient BCAO was elicited by inflating the occluders with air to $280 \mathrm{mmHg}$ for $2 \mathrm{~min} .5 \mathrm{~min}$ after the termination of the first $\mathrm{BCAO}, \mathrm{FiO}_{2}$ was reduced to 0.1 . Two more BCAOs $(2-2 \mathrm{~min})$ were elicited at the $5^{\text {th }}$ and $30^{\text {th }}$ minutes of hypoxic period. Five min after the last occlusion period, the animals were reoxygenated by ventilating them again with air for $5 \mathrm{~min}$, followed by $7 \mathrm{~min}$ asphyxia induced by halting artificial ventilation and blocking the endotracheal tube. After asphyxia, the animals were reventilated with air for 5 min then the anesthetized animals were euthanized to obtain the biological zero measurements. In group LSCI+ASPH $(n=5)$, cortical blood flow $(\mathrm{CoBF})$ velocity changes were assessed during asphyxic conditions: 20-minute asphyxia was induced (characterized in the following chapter) as in groups $\mathrm{ASPH}$ and $\mathrm{ASPH}+\mathrm{H}_{2}$ followed by room-air reventilation and short-term survival.

\section{PA/HIE experimental groups}

Animals for PA/HIE (groups CTR, ASPH, ASPH+H${ }_{2}$ ) were placed into a neonatal incubator (SPC 78-1; Narco Air-Shields, Inc., Hatboro, Pa., USA) in prone position then supplied with electrocardiograph (ECG) and electroencephalograph (EEG, Natus Neurology, Middleton, WI, USA) electrodes. Rectal temperature was measured continuously and kept in the physiologic range $\left(38.5 \pm 0.5^{\circ} \mathrm{C}\right)$ with a servo-controlled heating lamp. $\mathrm{O}_{2}$ saturation, MABP, HR and ECG were continuously monitored using a Hewlett-Packard M1094 monitor (Palo Alto, California, USA) and recorded online (MecifView, Arlington, Mass., USA). Prophylactic antibiotics were given iv.: penicillin $(50 \mathrm{mg} / \mathrm{kg} / 12 \mathrm{~h}$, Teva, Petah Tikva, Israel) and gentamicin $(2.5 \mathrm{mg} / \mathrm{kg} / 12 \mathrm{~h}$, Sanofi, Paris, France). Seizures were treated with 1-2 bolus injections of midazolam $(250 \mu \mathrm{g} / \mathrm{kg})$ [109]. The urinary bladder was tapped by suprapubic puncture at 12 hour of survival. Arterial blood samples ( $300 \mu \mathrm{l} / \mathrm{sample})$ were checked (ABL 5, Radiometer, Denmark or EPOC Blood Analysis, Epocal Inc., Ottawa Canada) at baseline, at the end of asphyxia, then at selected intervals up to 20 hours to keep blood gas values in the 
physiological range (Fig. 1). After the surgical procedure, an hour recovery period allowed stabilization of monitored physiological parameters prior obtaining baseline values. These parameters were recorded then during PA and for 10 minutes. After obtaining the baseline physiologic parameters, animals were divided into 3 groups: time control group ( $\mathrm{n}=7, \mathrm{CTR})$, asphyxia group $(\mathrm{n}=8, \mathrm{ASPH})$ and asphyxia $+\mathrm{H}_{2}$ treated group $\left(\mathrm{n}=8\right.$, ASPH $\left.+\mathrm{H}_{2}\right)$. Animals were randomized between groups ASPH and $\mathrm{ASPH}+\mathrm{H}_{2}$ by coin flip. PA in groups ASPH and $\mathrm{ASPH}+\mathrm{H}_{2}$ was induced by switching ventilation from medical air to a hypoxic-hypercapnic gas mixture $\left(6 \% \mathrm{O}_{2}, 20 \% \mathrm{CO}_{2}\right.$, balance $\left.\mathrm{N}_{2}\right)$ for 20 minutes, reducing the $\mathrm{RR}$ to $151 / \mathrm{min}$ and stopping the fluid/glucose administration. Piglets were reventilated (RR: 30 1/min) in group ASPH with medical air, whereas in group $\mathrm{ASPH}+\mathrm{H}_{2}$ with a gas mixture containing $\mathrm{H}_{2}$ gas $\left(2.1 \% \mathrm{H}_{2}, 21 \% \mathrm{O}_{2}\right.$, balance $\mathrm{N}_{2}$ ). In group $\mathrm{ASPH}+\mathrm{H}_{2}$, after 4 hours $\mathrm{H}_{2}$ treatment was stopped and ventilation with medical air was resumed.

\section{Electroencephalography recordings}

EEG activity in groups CTR, ASPH and $\mathrm{ASPH}+\mathrm{H}_{2}$ was recorded via subcutaneously inserted silver pin electrodes above the fronto-parietal and occipital regions. The impedance of all electrodes was checked to be below $5 \mathrm{k} \Omega$. Bandpass filters were applied between 1-70 $\mathrm{Hz}$, and an automatic noise filter (notch filter) rejected the $50 \mathrm{~Hz}$ component. EEG signal was amplified (Nicolet EEG v32, Natus Medical Inc, San Carlos, California, USA), recorded (sampling rate: $250 \mathrm{~Hz}$ ) and visualized online during the entire experiment with the manufacturer's software (NicoletOne). Data were stored on a hard disc of a personal computer. EEG activity was analysed offline with two approaches. First, 10 minute EEG epochs recorded at the beginning of each hour after PA were scored by a researcher unaware of the experimental group using an amplitude-based, incremental scoring system (Table 1) [110]. Briefly, continuous and high amplitude background activities $(>10 \mu \mathrm{V})$ were given lower scores while severely depressed and isoelectric activities $(<10 \mu \mathrm{V})$ received higher ones. In addition, if seizures appeared in the evaluated hour, 2 extra points were added. Second, EEG power spectrum analysis of the same EEG epochs was performed by the NicoletOne Review software using Fast Fourier Transformation (linear detrending, Hamming window; $\delta$ 1-4 Hz, $\theta 4-8 \mathrm{~Hz}, \alpha$ 8-12 Hz, $\beta$ 12-30 Hz) and absolute band powers were calculated. Total EEG power $\left(\mu \mathrm{V}^{2}\right)$ (summation of bands) values were selected to quantitatively characterize the recovery of brain electrical activity following PA and to complement the semiquantitative and the more observer-dependent scoring system. 


\begin{tabular}{c|c|} 
Score & Amplitude based EEG pattern \\
\hline 1 & $>25 \mu \mathrm{V}$ dominating pattern \\
2 & $>25 \mu \mathrm{V}$ with short durations $(1-5 \mathrm{sec})$ below \\
3 & $25-10 \mu \mathrm{V}$ \\
4 & $10-5 \mu \mathrm{V}$ \\
5 & Low voltage $(<5 \mu \mathrm{V})$ with bursts $(>25 \mu \mathrm{V}, 1-5 \mathrm{sec})$ \\
6 & $<5 \mu \mathrm{V}$ \\
7 & practically isoelectric \\
+2 & electro/clinical convulsion \\
\hline
\end{tabular}

Table 1. EEG activity above the occipital cortex was assessed visually at the end of asphyxia and during the initial $10 \mathrm{~min}$ of a given hour according to an amplitude-based scoring system, where low scores (1-3) represent predominantly high amplitudes $(>10 \mu \mathrm{V})$ and continuous EEG activity, whereas high scores (4-7) indicate severely depressed $(<10 \mu \mathrm{V})$ electrocortical functions. 2 extra points were added arbitrarily to the scored background activity if seizure occurred during the given timepoint.

5. $\mathrm{pH}_{\text {brain }}$ measurements

$\mathrm{pH}_{\text {brain }}$ measurements were performed inside a self-made Faraday cage in animals of group ASPH+pH $(n=13)$. Proton selective microelectrodes (PSM) were obtained from Unisense (Aarhus, Denmark), whereas saline-filled glass reference electrodes (RE, external tip diameter: $\sim 20 \mu \mathrm{m}$ ) were self-made then connected to $\mathrm{Ag} / \mathrm{AgCl}$ electrodes. The electrodes were mounted on stereotaxic manipulators, then the PSM was calibrated before each measurement in 3 different warmed buffer solutions ( $\mathrm{pH}: 6.10,7.10$, and 8.10, respectively). The heads of the animals were fixed in a stereotaxic frame and after retracting the scalp, two small circular craniotomies $(\varnothing \cong 5 \mathrm{~mm})$ were made over the fronto-parietal cortex, and the dura mater was gently removed. The PSM and the RE were each installed $\sim 2-2 \mathrm{~mm}$ deep into the exposed cortex, and a third $\mathrm{Ag} / \mathrm{AgCl}$ ground electrode was placed under the scalp. Potential difference between the PSM and the RE was amplified, converted to direct current (National Instruments, Austin, Texas, USA) then visualized and recorded online with the WinEDR freeware (author: John Dempster, University of Strathclyde, Glasgow, UK). Evaluation of the recordings was performed offline: by applying linear regression analysis, the signals from the calibration solutions were fitted with a curve and finally, the potential difference was converted and expressed on a $\mathrm{pH}$ scale. As the technique allows stable continuous $\mathrm{pH}_{\text {brain }}$ measurements reliably only for 3-4 hours, in different animals different time windows were chosen to be assessed (baseline, PA and the first 4 hours of survival, $8^{\text {th }}-14^{\text {th }}$ hours and $20^{\text {th }}-$ $24^{\text {th }}$ hours of survival as presented in the Results and also in Fig.1). After surgery, one-hour recovery period allowed stabilization of monitored physiological parameters prior obtaining the baseline values. 


\section{Histology analysis}

24 hours after the end of asphyxia, the brains were perfused with cold $\left(4^{\circ} \mathrm{C}\right)$ physiologic saline through the catheterized common carotid arteries in groups CTR, ASPH, $\mathrm{ASPH}+\mathrm{H}_{2}$ and $\mathrm{ASPH}+\mathrm{pH}$, respectively. Brains were gently collected and were immersionfixed in $4^{\circ} \mathrm{C}, 4 \%$ paraformaldehyde solution and further processed after two weeks. Paraffin embedded, $4 \mu \mathrm{m}$ sections were made from the frontal, temporal, parietal, occipital lobes. The haematoxylin-eosin stained slides were evaluated by a researcher blinded to the experimental groups with light microscopy (Leica Microsystems, Wetzlar, Germany). Damaged neurons were identified using the major hallmarks of dark eosinophilic cytosol, as well as pyknotic or disrupted nuclei. The degree of cerebrocortical neuronal damage was determined adapting a previously published scoring system (Table 2) [111] that allowed more comprehensive study of these large brain regions compared to the cell counting used in our previous studies [105]. Briefly, the pattern of neuronal injury (none < scattered < grouped < panlaminar) was determined in 20-20 non-overlapping fields of vision under 200x magnification in each assessed cortical region. Then, scores (0-9) were given to each region based on the frequency (\% of 20 examined fields) of the most severe pattern of injury observed. The neuronal damage in the ganglionic cell layer of the cerebellum, basal ganglia, thalamus and the hippocampal CA1 and CA3 regions was assessed with cell counting in non-overlapping areas (in 10, 5, 5, 3, 3 fields of vision respectively; under 200x magnification) as in [105]. The impact of asphyxia on cerebellum and subcortical brain regions was expressed as the percentage of damaged neurons. In order to be able to compare the severity of asphyxia-induced cortical neuronal damage at 24 hour of survival between the present new and our previously published [105] asphyxia method (20 min ventilation with $6 \% \mathrm{O}_{2} / 20 \% \mathrm{CO}_{2}$ versus 8 min suspension of ventilation), we re-analyzed the cortical samples from the ASPH group $(n=9)$ of the previous study using the scoring system as well, and we present the combined neuropathology scores in the Results for this group as well. 


\begin{tabular}{c|cc} 
Score & $\begin{array}{c}\text { Morphology of cortical } \\
\text { damage }\end{array}$ & $\begin{array}{c}\text { Ratio of the most severe } \\
\text { pattern per area }\end{array}$ \\
\hline 0 & \multicolumn{2}{|c|}{ No damage } \\
1 & \multicolumn{2}{|c|}{$<\%$} \\
2 & Scattered & $21-50 \%$ \\
3 & & $50 \%<$ \\
4 & & $<20 \%$ \\
5 & Grouped & $21-50 \%$ \\
6 & & $50 \%<$ \\
7 & & $<20 \%$ \\
8 & Panlaminar & $21-50 \%$ \\
9 & & $50 \%<$ \\
\cline { 2 - 3 }
\end{tabular}

Table 2. Cerebrocortical brain damage was evaluated by a graded, neuropathology scoring system, proposed by Foster et al. [111]. Briefly, 20-20 non-overlapping fields in the frontal, parietal, temporal and occipital cortices were checked in light microscopy under 200x magnification and the incidence (\% of the 20 examined areas) of most severe pattern (panlaminar $>$ grouped $>$ scattered $>$ no damage) of injury was expressed.

\section{8-hydroxy-2'-dezoxyguanosine (8-OHdG) immunohistochemistry}

Parietal cortex tissue microarrays from groups CTR, ASPH and $\mathrm{ASPH}+\mathrm{H}_{2}$ were produced using a custom-made stainless steel tissue puncher $(3 \mathrm{~mm})$ from the paraffin tissue blocks, sectioned at $4 \mu \mathrm{m}$, mounted on sylanized slides and processed for 8 -OHdG immunohistochemistry using a LEICA BOND-MAX automated immunostainer (Leica Microsystems). In the immunostainer, the slides were dewaxed at $72^{\circ} \mathrm{C}$, and antigen retrieval was performed at $\mathrm{pH}=6$. Then, slides were incubated with a 1:200 dilution of mouse monoclonal primary antibody against 8-OHdG (JaICA Inc., Fukuroi, Japan) for 20 min followed by horseradish peroxidase-conjugated rabbit anti-mouse secondary antibody for 15 minutes. 3,3'-diaminobenzidine was used to visualize the immunostaining, then the slides were counterstained with haematoxylin, to visualize the cell nuclei. The slides were covered with a coverslip then scanned in a slide scanner (Pannoramic MIDI, 3DHISTECH Ltd., Budapest, Hungary), and visualized on a personal computer using the Pannoramic Viewer software (3DHISTECH Ltd.) at 40x magnification. Homogenous, strong nuclear 8-OHdG immunoreactivity was considered as a sign of oxidative damage and the ratio of such nuclei to the total number of cell nuclei was determined and presented in the Results.

\section{Neuron specific enolase (NSE) ELISA}

$1 \mathrm{ml}$ arterial blood samples were taken from groups CTR, ASPH and $\mathrm{ASPH}+\mathrm{H}_{2}$ at regular intervals (baseline, 4 and 20 hours of survival) into EDTA-coated eppendorf tubes supplemented with $40 \mu \mathrm{l}$ protease inhibitor cocktail procured following the manufacturer's directions (cOmplete, EDTA-free Protease Inhibitor Cocktail, Roche Diagnostics Gmbh, 
Mannheim, Germany). The blood samples were centrifuged at $2200 \mathrm{~g}$ and $4^{\circ} \mathrm{C}$ for 5 minutes (Model 5418R, Eppendorf, Hamburg, Germany) and the plasma was transferred to fresh microcentrifuge tubes and stored at $-80^{\circ} \mathrm{C}$. Blood plasma NSE levels were determined using a commercially available, porcine-specific sandwich ELISA kit (Elabscience Biotechnology Co., Ltd., Wuhan, China). The measurements were performed in duplicates following the manufacturer's instructions using a microplate reader (Fluostar Optima, BMG LABTECH, Gmbh, Ortenberg, Germany) at $\lambda=450 \mathrm{~nm}$.

9. Statistical analysis

Results were analysed offline and plotted using SigmaPlot (v12.0, Systat Software Inc., San Jose, CA., USA). Vital parameters (core temperature, saturation, HR and MABP), arterial blood gas and metabolic parameters, cell counts, $\mathrm{pH}_{\text {brain }}$, plasma NSE levels, relative cortical as well as arteriolar blood flow velocity changes and pial arteriolar diameters were expressed as mean \pm S.E.M. EEG scores, EEG total power and neuropathology scores were expressed as median, $25^{\text {th }}-75^{\text {th }}$ and $5^{\text {th }}-95^{\text {th }}$ percentiles. Normality was tested with the ShapiroWilk test. Parametric data were compared with one-way- or two-way repeated measure of analysis of variance (RM ANOVA) followed by the Student-Newman-Keuls post hoc test. For non-parametric data RM ANOVA on ranks and for pairwise comparisons the StudentNewman-Keuls post hoc test was applied. Level of significance (p) was set at 0.05. 


\section{RESULTS}

1. The effects of BCAO on CoBF velocity under different ventilation conditions.

Prior the onset of hypoxic ventilation, vital parameters of the animals (group LSCI+BCAO) were in the respected physiological ranges and did not differ significantly from the other experimental groups at baseline (core temperature: $38.5 \pm 0.1^{\circ} \mathrm{C}, \mathrm{O}_{2}$ saturation: 94 $\pm 1 \%$, MABP: $79 \pm 3 \mathrm{mmHg}$ and HR: $163 \pm 11$ 1/min).

Hypoxia resulted in acidosis $\left(\mathrm{pH}_{\mathrm{a}}\right.$ : from $7.42 \pm 0.03$ to $\left.7.29 \pm 0.04\right)$, hypoxemia $\left(\mathrm{P}_{\mathrm{a}} \mathrm{O}_{2}\right.$ : from $79 \pm 8$ to $22 \pm 2 \mathrm{mmHg})$ but normocapnia $\left(\mathrm{P}_{\mathrm{a}} \mathrm{CO}_{2}: 37 \pm 2 \mathrm{mmHg}\right)$ was retained. It is noteworthy, that 5 out of 7 animals maintained MABP throughout the hypoxic insult (MABP: from $78 \pm 12$ to $85 \pm 19 \mathrm{mmHg}$ ) but moderate hypotension occurred in 2 piglets. Then, room air ventilation restored blood gas values (data not shown). The subsequently induced asphyxia elicited more severe acidosis $\left(\mathrm{pH}_{\mathrm{a}}: 6.73 \pm 0.02\right)$, hypoxemia $\left(\mathrm{P}_{\mathrm{a}} \mathrm{O}_{2}: 14 \pm 3 \mathrm{mmHg}\right)$ and hypercapnia $\left(\mathrm{P}_{\mathrm{a}} \mathrm{CO}_{2}: 87 \pm 6 \mathrm{mmHg}\right)$.

The $1^{\text {st }}$ BCAO performed during normoxic ventilation (Fig.2 Panel A) did not influence baseline parenchymal perfusion. During hypoxic ventilation, progressive increases in CoBF velocity were observable in animals maintaining MABP during the insult (Fig.2 Panel B) and interestingly, the effects of the $2^{\text {nd }}$ and $3^{\text {rd }}$ BCAO on cerebrocortical microcirculation were not detectable. In addition, in hypotensive animals $(n=2 / 7)$ at $\sim 10 \mathrm{~min}$ after the onset of hypoxemia cortical perfusion decreased to $63 \%$ and $62 \%$ of baseline, respectively.
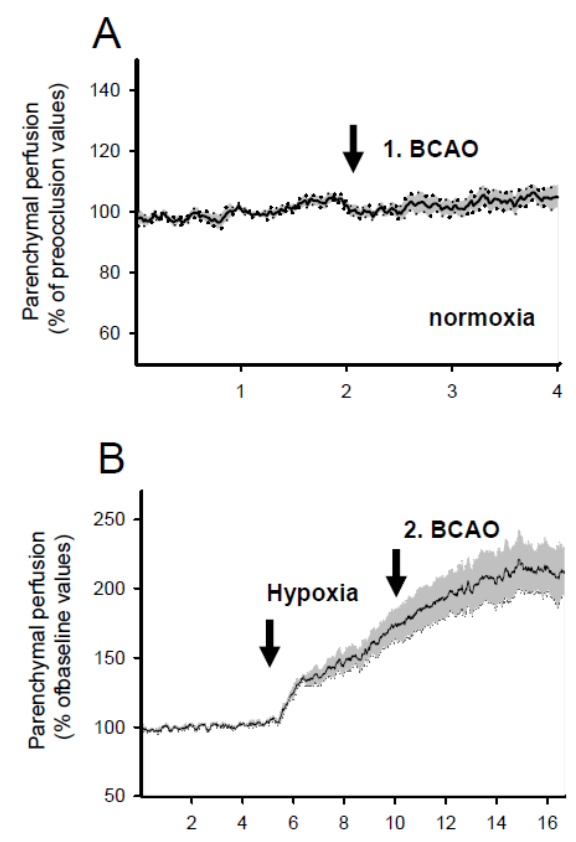
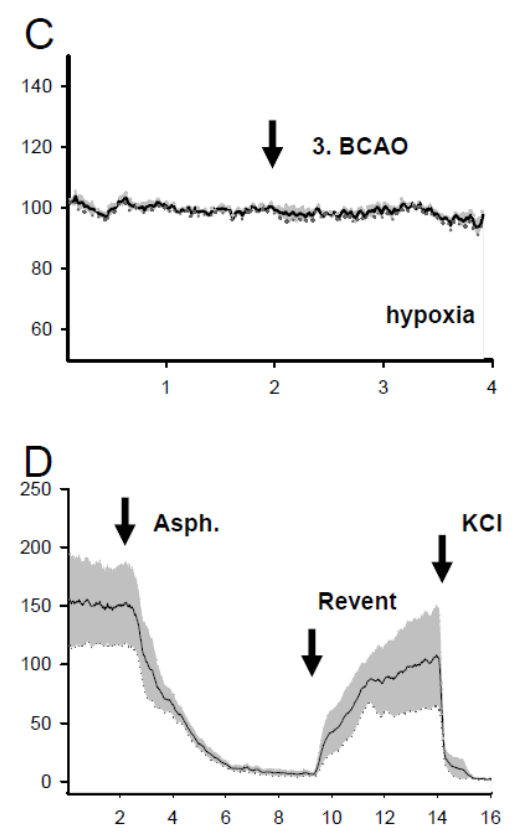

Figure 2. CoBF changes in newborn piglets obtained from LASCA. Panel A introduces baseline (100\%) CoBF. $\mathrm{BCAO}$ at $2 \mathrm{~min}$ did not influence parenchimal perfusion. Hypoxic ventilation elicited hyperemic response in the cortical microcirculation, however, it is clearly seen that BCAOs at the beginning (Panel B) or at the end of hypoxia (Panel C) unaffected CoBF. Finally, asphyxia (Panel D) resulted in severe cerebrocortical ischemia, indicated by the drop in CoBF that was restored to baseline again prior euthanasia $(\mathrm{KCl})$. CoBF data expressed as \% change from baseline, recorded before the $1^{\text {st }} \mathrm{BCAO}$, and presented as mean \pm S.E.M. (black line and grey shaded areas, respectively). 
Room air reventilation elicited a reactive hyperaemic response in parenchymal perfusion. Asphyxia, in contrast to hypoxia triggered severe reductions in cortical perfusion, however this ischemia was also reversible upon reventilation (Fig.2 Panel D).


Figure 3. Representative laser speckle contrast images (LSCI) obtained through a closed CW from a piglet. Scale bars on the right (top for Panels A and D, middle for Panels B and E and bottom for Panels C and F) represent the contrast values: higher and lower blood flow velocities (e.g. in the arterioles and the parenchima, respectively) are indicated by the lower and the higher contrast values, respectively. Panel A represents the baseline condition. BCAO did not influence markedly the speckle map on Panel B. $10 \mathrm{~min}$ (Panel C) and $30 \mathrm{~min}$ (Panel D) after the onset of hypoxic ventilation, sustained hyperemic response was observed, moreover, substantial pial arterial vasodilation on both pixel maps can be clearly seen. Arteriolar and cortical blood flow velocity decreased significantly during asphyxia (Panel E). Finally, euthanasia resulted in increased speckle contrast (Panel F) with the simultaneous disappearance of the perfusion map. 
BCAO during normoxic ventilation did not influence pial arteriolar diameter (Fig.4 Panel A), although marked increase in arteriolar blood flow velocity was observable. Furthermore, significant vasodilation was detectable during hypoxia along with remarkable elevations in pial arteriolar blood flow velocities (Fig.4 Panel B). Interestingly, similar to parenchymal perfusion, the analysed parameters were largely unaffected by BCAO. Finally, asphyxia elicited a significant drop $(\sim 90 \%)$ in arteriolar blood flow reversed upon reventilation.
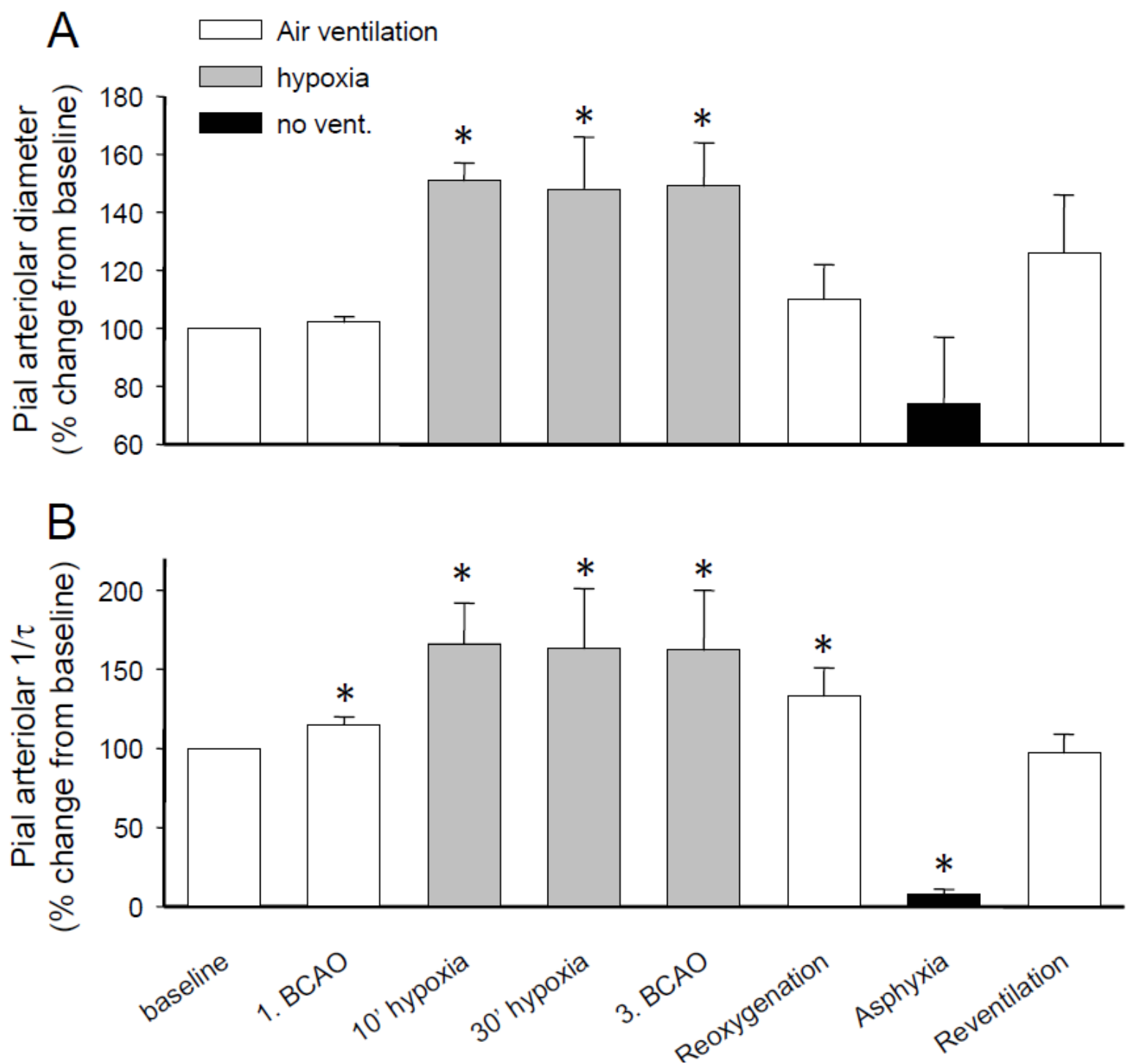

Figure 4. The effects of mechanical vessel occlusion on pial arteriolar diameter (Panel A) and $1 / \tau$ changes (Panel B) during different ventilation conditions. The $1^{\text {st }}$ BCAO performed under normoxic ventilation did not influence baseline pial arteriolar diameters. Hypoxic ventilation elicited significant vasodilation in the examined pial vessels along with marked increase in arteriolar blood flow velocity changes, indicated in the $10^{\text {th }}$ and $30^{\text {th }}$ minute of the stimulus. BCAO during hypoxia unaffected pial arteriolar responses. Reoxygenation restored the diameters of the vessels, however, blood flow velocity remained still elevated during the short observation period. Pial arteriolar blood flow velocity was remarkably ceased by the end of asphyxic insult (clamped endotracheal tude and suspended artificial ventilation). Upon room air reventilation, the examined hemodynamic parameters were corrected. Data presented as mean \pm S.E.M. $\mathrm{p}<0.05 . * v s$. baseline. 


\section{Characterization of the PA stress}

The normoxic time control animals (group CTR) showed physiologic vital parameters (core temperature, $\mathrm{HR}, \mathrm{MABP}$ and $\mathrm{O}_{2}$ saturation), blood gas values along with blood glucose levels during the whole experiment (Fig.7), according to values obtained from newborn piglets during the first days of life, reported by Randall et al. [77]. Nevertheless, baseline physiologic and blood gas values of groups $\mathrm{ASPH}$ and $\mathrm{ASPH}+\mathrm{H}_{2}$ did not differ from time control animals as well (Fig.7).

20 min asphyxia induced a biphasic response in HR and MABP values: after the onset of asphyxia, $\mathrm{O}_{2}$ saturation fell rapidly while $\mathrm{HR}$ and MABP increased simultaneously, remaining elevated for minutes during the insult. Then HR along with MABP decreased uniformly in all asphyxiated animals till the end of asphyxia (Fig.5). Interestingly, severe hypotension (MABP $<50 \mathrm{mmHg}$ ) did not develop in the asphyxiated animals.
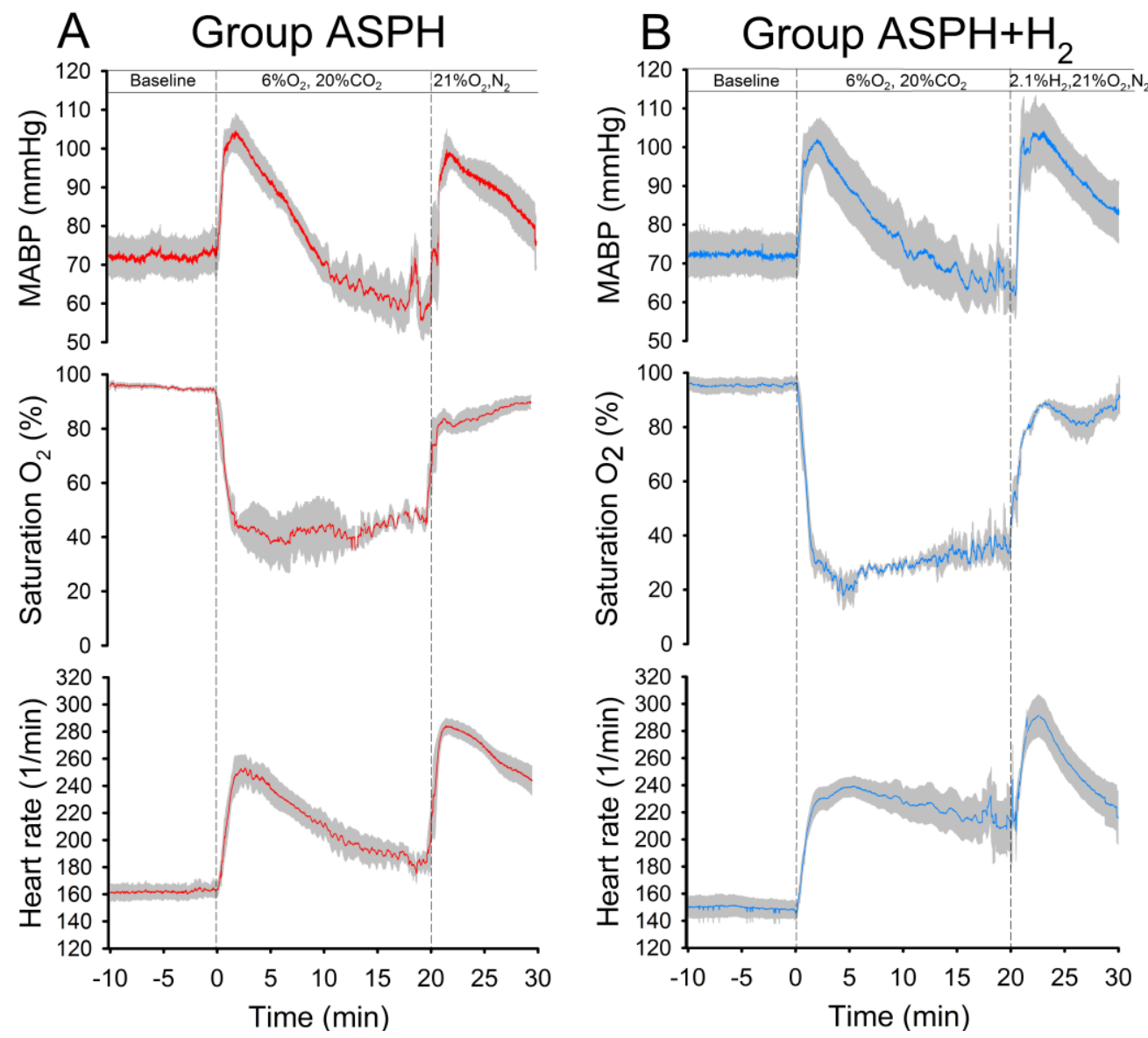

Figure 5. Hemodynamic responses during experimental asphyxia and early reventilation. Baseline vital parameters did not differ among experimental groups (ASPH and $\mathrm{ASPH}+\mathrm{H}_{2}$ ). 20 min hypoxic-hypercapnic ventilation elicited a biphasic response in mean arterial blood pressure (MABP, top panels) and heart rate (bottom panels) as $\mathrm{O}_{2}$ saturation (middle panels) fell. Reventilation from asphyxia elicited a second elevation in MABP and heart rate that returned gradually to baseline levels afterwards (data not presented). Data shown as mean \pm S.E.M. (colored lines and grey shaded areas, respectively). 
From metabolic perspective, our hypoxic-hypercapnic stress resulted in severe acidosis, hypercapnia and hypoxemia along with marked elevations in blood glucose (Fig.7 Panels E-H). In addition to the hypercapnia-induced respiratory acidosis, base excess was reduced by $17.4 \pm 5.0 \mathrm{mmol} / \mathrm{l}$, blood lactate level rose significantly (from $1.58 \pm 1.02 \mathrm{mmol} / \mathrm{l}$ to $10.30 \pm 2.51 \mathrm{mmol} / \mathrm{l})$ that remained still elevated $(6.42 \pm 2.39 \mathrm{mmol} / \mathrm{l})$ at 1 hour of survival then returned to baseline $(0.82 \pm 0.22 \mathrm{mmol} / \mathrm{l})$ by 4 hour (data from group ASPH+pH). The severe reduction in $\mathrm{O}_{2}$ saturation, monitored by pulse oximetry, was confirmed by a significant drop in central $\mathrm{O}_{2}$ saturation as well, obtained from arterial samples (from $94 \pm 5 \%$ to $13 \pm 4 \%$, representative data from group $\mathrm{ASPH}+\mathrm{pH})$.

The EEG became isoelectric within 1-2 minutes after the onset of asphyxia and it is noteworthy that CoBF increased (Fig.6) and remained elevated during the asphyxic insult (group ASPH+LSCI).

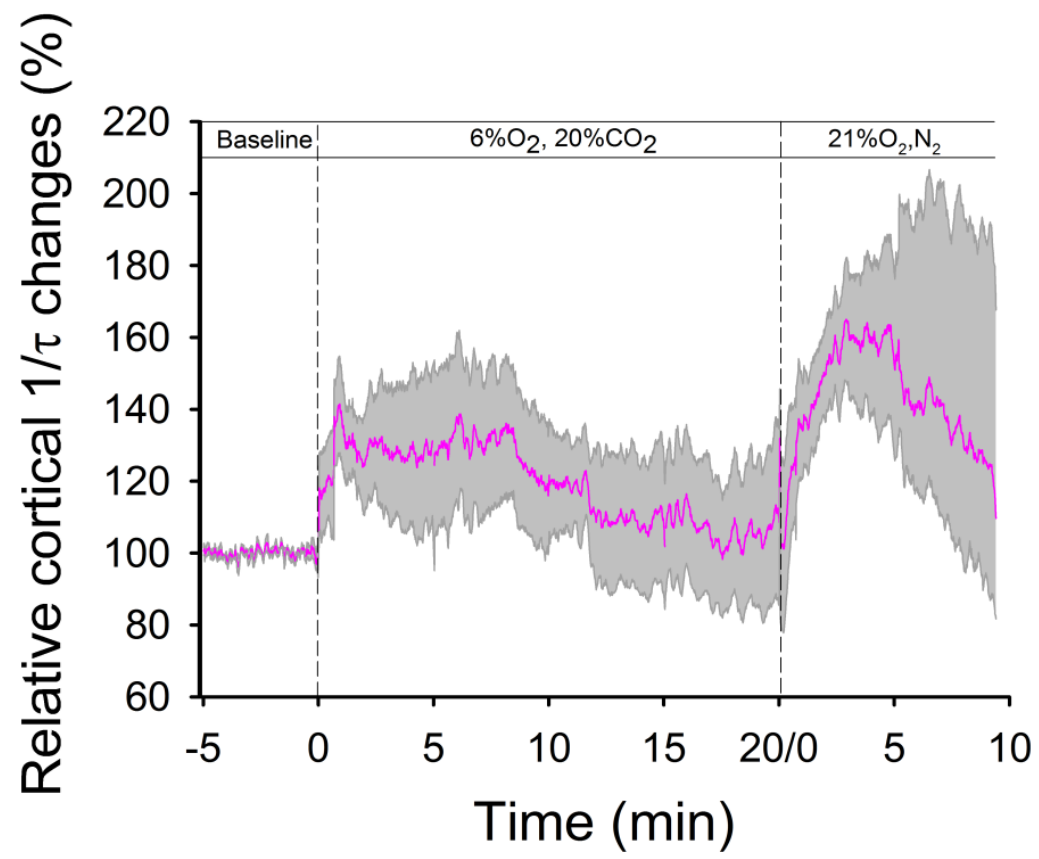

Figure 6. CoBF velocity changes during asphyxia. After the onset of asphyxia, CoBF rose moderately and remained elevated during the 20 min period. Notably, cerebral ischemia did not develop during the insult. Room air reventilation resulted in a second, reactive hyperemic response in the experimental animals (group ASPH+LSCI, n=5). Data presented as mean \pm S.E.M. (purple solid line, grey shaded areas; respectively). 



Figure 7. Vital parameters (core temperature $\left[\right.$ Panel A], $\mathrm{O}_{2}$ saturation $[$ Panel $\mathrm{B}]$, heart rate $[$ Panel $\mathrm{C}]$ and mean arterial blood pressure [MABP, Panel D]) were continuously monitored and recorded at baseline (B), in the last minute of asphyxia (A) and during the survival period. Arterial blood samples were checked regularly for $\mathrm{pH}$ (Panel E), $\mathrm{pCO}_{2}$ (Panel F), $\mathrm{pO}_{2}(\mathrm{Panel} \mathrm{G})$ and glucose (Panel H) to maintain metabolic parameters in the respected physiologic range after PA. Vital and blood metabolic parameters prior the asphyxic insult were identical between groups and remained in physiologic ranges during the observation period in the time control animals (CTR, $n=7$, green). Asphyxia elicited comparable and significant degree of hypoxemia (Panels B and G), hypercapnia (Panel F), acidosis (Panel E) and hyperglycemia in groups ASPH ( $n=7$, red) and $\mathrm{ASPH}+\mathrm{H}_{2}(\mathrm{n}=7$, blue) that were restored to preasphyxic levels with reventilation. Data presented up to 8 hours as animals did not show marked alterations in these parameters during the remaining observation period. However, animals of group ASPH required higher $\mathrm{FiO}_{2}$ to maintain acceptable saturation resulting in modestly elevated $\mathrm{P}_{2} \mathrm{O}_{2}$ during the early survival period (Panel G). In addition, HR changes in this group were found to be significantly higher vs. time control (CTR) animals (Panel C). Data are presented as mean \pm S.E.M. $\mathrm{p}<0.05 . * v s . \mathrm{B}, \uparrow v s . \mathrm{CTR}$ and $\S v s . \mathrm{ASPH}+\mathrm{H}_{2}$ at given time point. 
Finally, $\mathrm{pH}_{\text {brain }}$ decreased progressively and robustly during the hypoxic-hypercapnic insult from $7.08 \pm 0.02$ to $5.75 \pm 0.27$ (Fig.8 Panel A) that largely exceeded the $\mathrm{pH}$ drop in the arterial blood $\left(\mathrm{pH}_{\mathrm{a}}\right.$ : from $7.53 \pm 0.27$ to $\left.6.79 \pm 0.07\right)$ in this group.
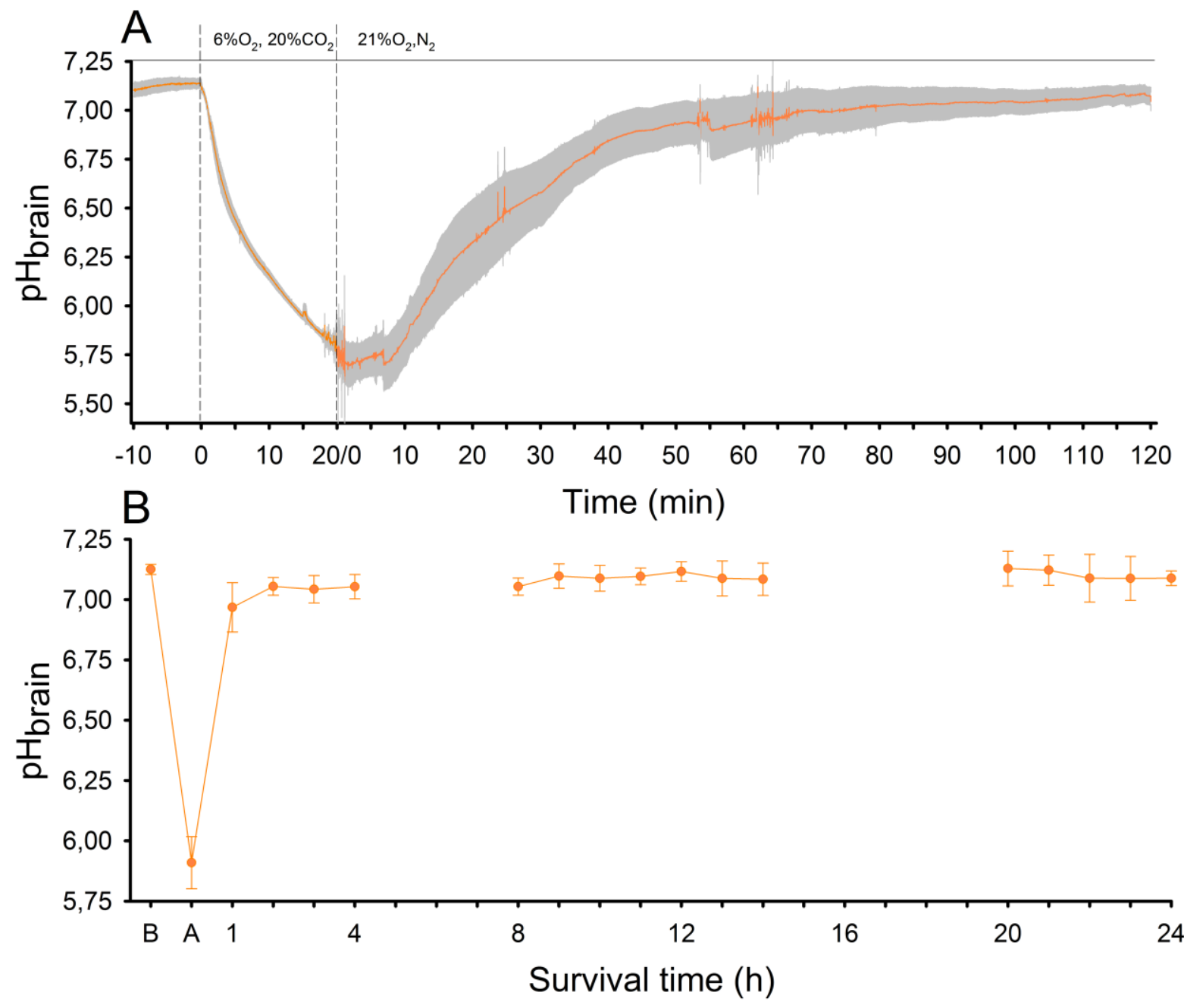

Figure 8. $\mathrm{pH}_{\text {brain }}$ changes during asphyxia (A) and the subacute phase of HIE in our large animal PA model. Baseline (B) physiologic and blood metabolic parameters did not differ significantly from the other ASPH

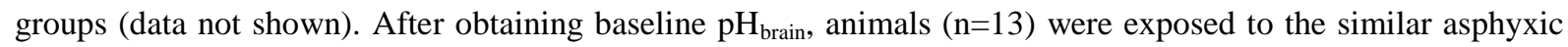
insult compared to the other ASPH groups. $\mathrm{pH}$ in the cerebral cortex dropped uniformly during the insult ( $\mathrm{n}=5$ recordings) that returned gradually to preasphyxic level by 1-2 hours under room air reventilation (Panel A). During the observation period (number of recordings: $n=8$ between 8 -14 hours, $n=3$ between 20-24 hours), $\mathrm{pH}_{\text {brain }}$ remained in the physiologic range and did not show significant alterations (Panel B). Data are presented as mean \pm S.E.M. on Panels A and B (solid line with grey shaded areas and dots with error bars; respectively). 


\section{Characterization of the HIE development}

Cardiovascular instability in 1-1 piglets (at 8 and 12 hours, respectively) of groups $\mathrm{ASPH}$ and $\mathrm{ASPH}+\mathrm{H}_{2}$ resulted in a $12.5 \%$ (2 out of 16 animals) mortality rate, consequently, data of these animals were excluded from the results.

Reventilation from asphyxia, irrespective of the composition of the gas mixture, resulted in a quick restoration of $\mathrm{O}_{2}$ saturation followed by uniform elevation in $\mathrm{HR}$ and MABP in all experimental groups. Nevertheless, room air reventilation elicited an immediate reactive hyperaemic response in $\mathrm{CoBF}$ changes revealed in group LSCI+ASPH. Core temperature, $\mathrm{MABP}$ and $\mathrm{O}_{2}$ saturation remained in the respective physiologic ranges during the entire experiment and did not show statistical difference between groups. Interestingly, only HR was markedly elevated in group ASPH and statistically different from time control animals.

Although central $\mathrm{O}_{2}$ saturation along with arterial $\mathrm{pH}, \mathrm{pCO}_{2}$ and $\mathrm{O}_{2}$ returned to baseline values by 1 hour of reventilation, blood glucose with lactate levels were still elevated that time point. By 4 hour, blood metabolites were also restored and remained in the normal range along with blood gas values for the remaining time of the experiment. Some piglets (5:7:4 in groups CTR, $\mathrm{ASPH}$ and $\mathrm{ASPH}+\mathrm{H}_{2}$; respectively) required higher $\mathrm{FiO}_{2}$ to maintain adequate $\mathrm{O}_{2}$ saturation resulting in significantly elevated $\mathrm{P}_{a} \mathrm{O}_{2}$ in group ASPH during early reventilation.

Brain electric activity was gradually restored in piglets, reventilated with room air containing $2.1 \% \mathrm{H}_{2}$ (group $\mathrm{ASPH}+\mathrm{H}_{2}$ ), resulting in lower EEG scores and statistically higher total EEG power, whilst EEG remained isoelectric in 5/7 animals in group ASPH (Fig.9). It is noteworthy, that 2 animals in group ASPH manifested electro-clinical convulsions at 9 and 13 hour of survival, identified by repetitive trunk/limb muscle contractions with characteristics EEG patterns. 

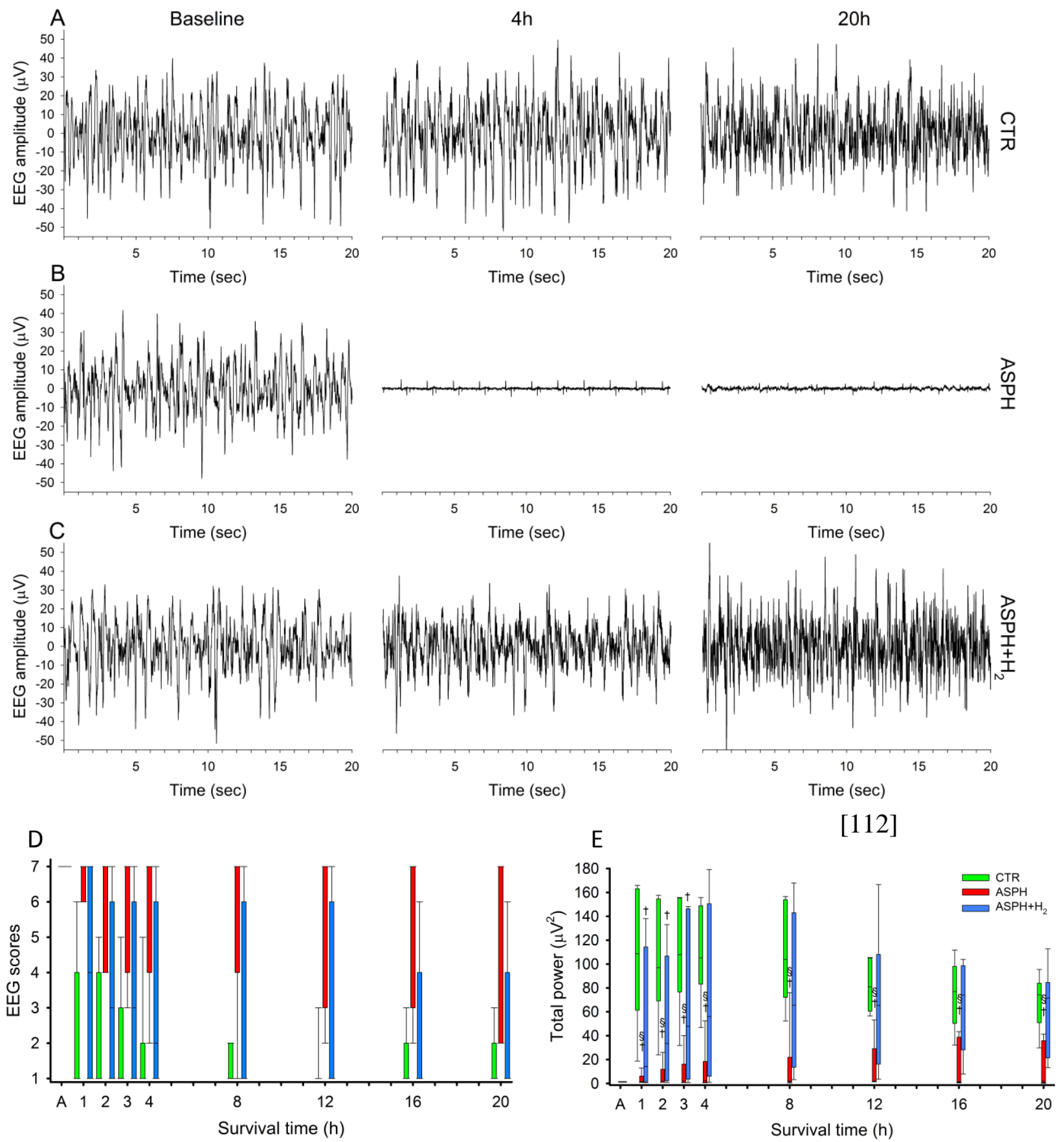

Figure 9. Cerebrocortical activity changes during the observation period. Raw EEG recordings over the occipital cortex (Panels A-C) represent the dominating brain electric activity at given timepoints in the experimental groups. Time control animals (CTR) manifested continuous EEG activity during the survival period, resulting in low EEG scores (Panel D) along with high total EEG power (Panel E). Asphyxia severely depressed EEG activity in group ASPH and most animals manifested isoelectric brain activity with mechanical ventilation related artefacts (Panel B), corresponding high EEG scores and low total power. Molecular $\mathrm{H}_{2}$ reventilation restored brain electric activity (group ASPH $+\mathrm{H}_{2}$, Panel C) and total power values did not differ significantly from time control animals at given timepoints. Data presented as median, $25^{\text {th }}-75^{\text {th }}$ and $5^{\text {th }}-95^{\text {th }}$ percentiles (line, box, error bars). $p<0.05 . \dagger v s$. CTR, $\S v s$. $\mathrm{ASPH}+\mathrm{H}_{2}$. 
PA did not induce elevations in plasma NSE levels that were $96.2 \pm 6.7 \%$ and $106.1 \pm 36 \%$ of baseline levels at 4 and 20 hour of survival, respectively (group ASPH, $n=6$ ).

Furthermore, $\mathrm{pH}_{\text {brain }}$ was gradually restored within 1-2 hours (Fig.8) under room air reventilation and marked $\mathrm{pH}_{\text {brain }}$ alterations were not observed during the subsequent survival period (between 8-14 and 20-24 hours).

\section{Neuropathology results}

Histology analysis of time control animals (group CTR) revealed minimal brain injury resulting from the low-frequency occurrence of scattered damaged neurons (Fig.10). Asphyxia induced extensive brain damage by 24 hour of survival, indicated by laminar or even confluent panlaminar necrotic areas in the cortical areas and by the high incidence of damaged neurons in other brain regions. Molecular $\mathrm{H}_{2}$, administered during the first 4 hour of reventilation, alleviated the cortical damage and preserved neurons also in other examined (e.g. cerebellum, thalamus, basal ganglia and hippocampus) areas as well.

Then, we applied the neuropathology scoring system on the asphyxia group from our previously reported PA/HIE swine model (group ASPH, n=9, clamped endotracheal tube with suspended ventilation [105]), in order to be able to compare the degree of cortical neuronal damage between the two PA methods. We found that the summated cortical histopathology scores of that group were significantly smaller than the values of our current new PA/HIE model $\left(17 ; 10 ; 21\right.$ versus $32 ; 20 ; 36$; median; $25^{\text {th }}-75^{\text {th }}$ percentiles, respectively). We conclude that our new model could elicit more robust cortical damage compared to the previously employed PA method.

In addition, 8-OHdG immunohistochemistry analysis revealed intensive nuclear staining in the parietal cortex and the hippocampal CA1 subfield in the asphyxiated animals (group ASPH). $2.1 \% \mathrm{H}_{2}$ reventilation (group ASPH $+\mathrm{H}_{2}$ ) reduced oxidative stress indicated by the lower incidence of immunopositive nuclei at 24 hour of survival (Fig.11). 

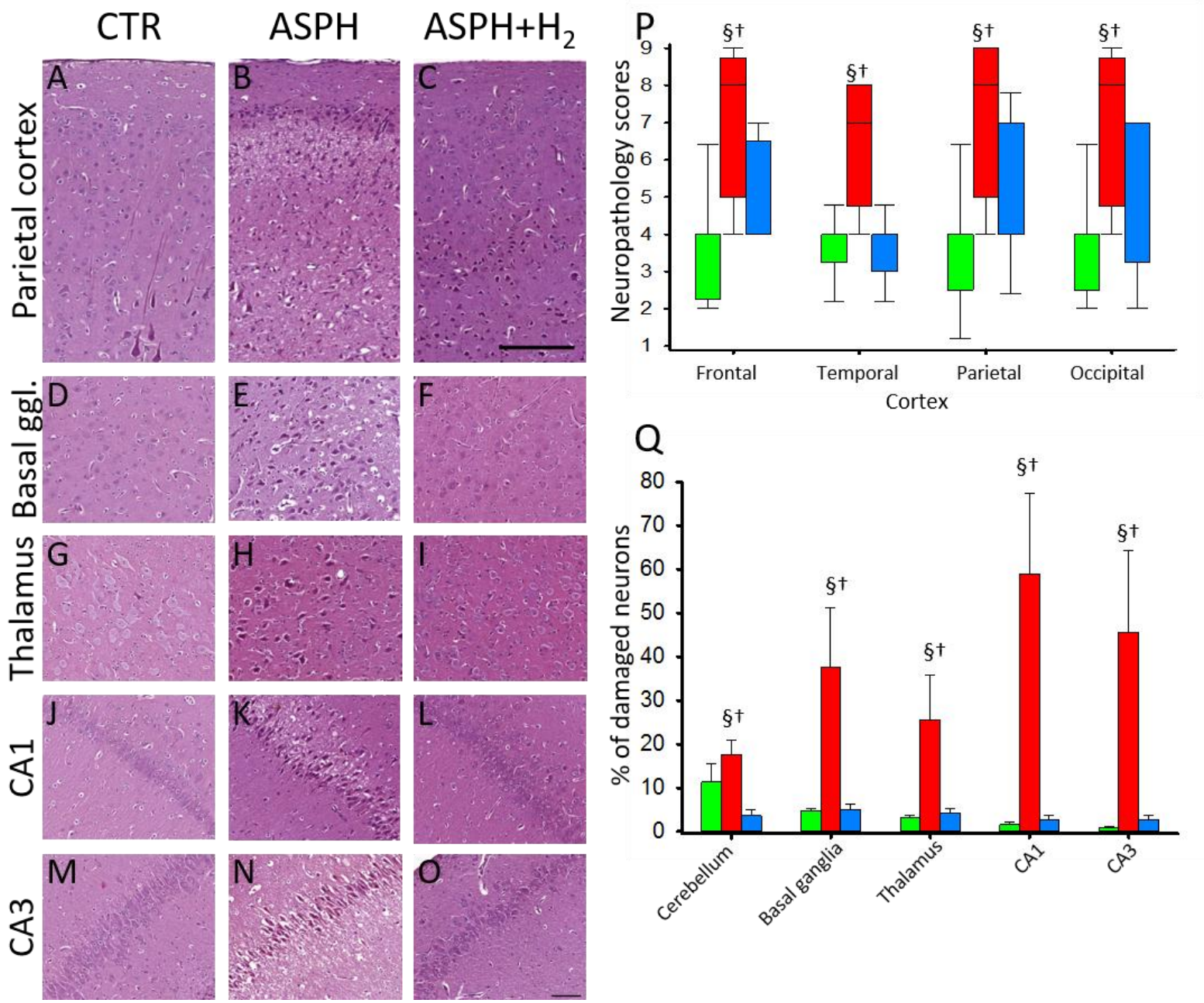

Figure 10. $\mathrm{H}_{2}$ reventilation mitigates PA-induced neonatal brain damage. Representative photomicrographs from the parietal cortex (Panels A-C), basal ganglia (Panels D-F), thalamus (Panels G-I) along with hippocampal $\mathrm{CA} 1$ and $\mathrm{CA} 3$ regions (Panels $\mathrm{J}-\mathrm{O}$ ) demonstrate the degree of neuronal injury, quantitatively expressed by neuropathology scores in cortical regions (Panel P) and by cell counting in various other brain areas (Panel Q). Neuropathology analysis revealed low incidence of damaged neurons in time control animals (CTR, green), indicated also by low histology scores. Experimental asphyxia in group ASPH (red) elicited more extensive often panlaminar cortical damage in all examined lobes, resulting in higher scores. Similarly, the proportion of damaged neurons were higher in the other assessed areas. Reventilation (4h) with $2.1 \% \mathrm{H}_{2}$ alleviated neonatal brain damage, marked by lower histology scores along with preserved brain areas. Scale bar: $100 \mu \mathrm{m}$ for top (AC) and $50 \mu \mathrm{m}$ for the other (D-O) panels. Data presented as median, $25^{\text {th }}-75^{\text {th }}$ and $5^{\text {th }}-95^{\text {th }}$ percentiles (line, box, error bars) on Panel P and as mean \pm S.E.M. on Panel Q. $\mathrm{p}<0.05$. $\dagger$ vs. CTR, $\S v s . \mathrm{ASPH}+\mathrm{H}_{2}$. 



Figure 11. Molecular $\mathrm{H}_{2}$ affords neuroprotection via reducing oxidative stress after PA. Immunohistochemistry analysis revealed low incidence of $8-\mathrm{OHdG}$ immunopositive nuclei (Panels A and D) in the time control animals (CTR). The 20 min asphyxia increased the ratio of strong immunopositive neurons in the examined areas: in the hippocampal CA1 (Panel B) and parietal cortex (Panel E). 2.1\% $\mathrm{H}_{2}$ resuscitation markedly attenuated oxidative stress (Panels $\mathrm{C}$ and F), indicated by the lower proportion of stained nuclei against 8-OHdG. Scale bars: $50 \mu \mathrm{m}$ and $100 \mu \mathrm{m}$ on Panels $\mathrm{C}$ and F, respectively. Data on Panels $\mathrm{G}$ and $\mathrm{H}$ are expressed as mean \pm S.E.M. $\mathrm{p}<0.05$. $\dagger v s . \mathrm{CTR}, \S v s$. ASPH $+\mathrm{H}_{2}$. 


\section{DISCUSSION}

The major findings of the studies were the followings:

1) BCAO per se does not influence cerebrocortical perfusion and pia arteriolar blood flow velocity during different ventilation (normoxic and hypoxic) conditions in newborn piglets.

2) We introduced a novel PA/HIE newborn piglet model. By characterizing our model, we demonstrated all the major metabolic hallmarks of PA (hypoxemia, hypercapnia with mixed acidosis) to be present in a translationally relevant degree. Furthermore, we showed that no significant cortical ischemia developed. Although the reventilation restored blood gases along with the metabolic (e.g. glucose, lactate) parameters within hours, HIE development was clearly demonstrated on one hand by the severely depressed brain electrical activity throughout the observation period and on the other hand by the detection of extensive neuronal damage revealed by neuropathology analysis.

3) We successfully employed ion-selective electrodes to study $\mathrm{pH}_{\text {brain }}$ over the subacute phase of HIE development in a large animal model. We found that (1) $\mathrm{pH}_{\text {brain }}$ dropped to extremely low values during asphyxia exceeding the blood acidosis by more than 1 $\mathrm{pH}$ unit, (2) after the gradual restoration of $\mathrm{pH}_{\text {brain }}$ after asphyxia, there were no secondary $\mathrm{pH}_{\text {brain }}$ alterations despite the development of HIE at least during the 24hour survival period.

4) Administration of molecular $\mathrm{H}_{2}$ starting after PA but within the suggested therapeutic window (1-4 hours) afforded neuroprotection assessed in the subacute phase ( $1^{\text {st }}$ day) of HIE, indicated by the restoration of brain electric activity and the better preservation of neuronal viability in various cortical and subcortical brain areas. Moreover, $\mathrm{H}_{2}$ exerted its neuroprotection likely through alleviation of the asphyxiainduced oxidative stress shown by the reduction of $8-\mathrm{OHdG}$ immunopositive neuronal nuclei in the assessed regions: the parietal cortex and the hippocampal CA1 subfield.

One of the greatest successes in human history was the halving of global NMR due to an international effort thus fulfilling this Millennium Developmental Goal in 15 years [3]. According to the 2015 UNICEF report NMR declined to 20 out of 1000 live births globally since 1990, however, it is still high in less developed areas as in Sub-Saharan Africa and Oceania [113]. Hence, since 2015, new strategies have been implemented to reduce 
preventable neonatal death and thus to further lower NMR below 10 out of 1000 live births globally, by 2035 [114].

$\mathrm{NE}$ is complex clinical syndrome of the term neonate, characterized by impaired neurologic functions during early life. HIE is regarded as a subset of NE, however, the terms of NE and HIE are still often used imprecisely or synonymously in the literature, hence, many clinical reviews with editorial comments suggest the use of HIE in the certainty of intrapartum asphyxia $[14,15,19,115]$. The inaccurate application of the nomenclatures for many years resulted in the inability to assess the exact incidence of HIE retrospectively, although NE, irrespective for aetiology, is estimated for 1-6 out of 1000 live births globally [116]. Approximately $15-20 \%$ of the affected infants die during the neonatal period whilst 20 $25 \%$ suffer permanent neurological sequelae [117].

PA and HIE are significant contributors to neonatal mortality and morbidity worldwide. Asphyxia-related neonatal loss occurs predominantly ( 98\%) on the first week of life, particularly during the first 72 hours ( 75\%) [4]. Victims of asphyxia can recover completely, nevertheless, variable neuropsychiatric syndromes may develop later as long term effects of mild asphyxiation, e.g. attention deficit disorder, hyperactivity or even schizophrenia [118]. Furthermore, the infants manifesting symptoms of HIE are also at great risk for the development of seizures and $\mathrm{CP}$, resulting in life-long neurodevelopmental disabilities. Beside the motor deficits characterizing $\mathrm{CP}$, persistent consequences of HIE may include cognitive and sensory impairments, learning problems, nutritional difficulties (e.g. feeding problems, recurrent aspiration pneumonia) along with musculoskeletal disorders (e.g. scoliosis, muscle atrophy, osteoporosis), resulting in limited life expectancies of survivors. Therefore, CP imposes great burden on families, societies and health care service as well [18]. From economic perspectives it is also noteworthy, that the life-long cost of CP per person is estimated to be approximately $\$ 900.000$ in the USA [119]. In addition, one of the parents will not able to work and to contribute to economy. Moreover, the HIE survivor children will also have poor employment prospects. Thus, prompt recognition of signs of fetal distress along with cost-effective therapeutic interventions are urgently warranted to rescue infants with suspected PA and to mitigate the life-long consequence of HIE.

PA and HIE are key areas of paediatric research. Severe perinatal respiratory distress can induce a chain of events in the newborn increasing the risk for permanent neonatal brain injury. To combat against the development of HIE and to improve the quality of lives of PA 
survivors, advanced strategies in resuscitation and neuroprotection, furthermore, innovative diagnostic tools must be successfully implemented into clinical practice. Preclinical animal experiments open the door to study the molecular mechanisms of HIE and to test neuroprotective approaches. Various neonatal animal models have been considered to be the substitutions of the human neonate. Establishing a PA/HIE animal model truly reproducing the human clinical entity with its all critical hallmarks is challenging because of many reasons. First, the exact clinical history and the mechanism of PA are heterogeneous, thus the cause, onset, duration and severity remain unclear in many cases. Second, the ongoing PA cannot usually be tracked directly (e.g. the degree and duration of hypoxia) but can rather be suspected from indirect signs of fetal distress (e.g. irregular HR patterns, meconium stained amniotic fluid). Third, there are no clear universally accepted criteria for the levels of severe hypoxemia and hypercapnia defining severe birth asphyxiation. Consequently, animal models remain always just approximation of the human clinical conditions and the interpretation of the results obtained from animal experiments must be applied carefully.

Small animal models (e.g. guinea pig, rat, rabbit and mouse) contributed undoubtedly to better understanding the molecular responses of cerebral hypoxia/ischemia. The merits of PA/HIE small animal models include easy availability and simple maintenance, low costs, and favorable ethical issues. However, the interpretation of the results for a term neonate must be done very carefully as significant differences in brain developmental stage, cerebral metabolism, and cardiovascular regulation exist. On the other hand, large animal models (e.g. sheep, lamb, goat, dog, monkey and pig) offer better instrumentation possibilities to more precisely regulate the physiology of the experimental animals and possess larger clinical relevancy, but their availability and the need for special laboratory equipment for intensive care can limit their routine use. Each major research group in the field of neonatology designed their own animal model to study the pathophysiology of PA/HIE, claiming their animal species/model is to be superior for testing possible hypotheses. Over the past five decades as our understanding of PA/HIE accumulated, the animal models have also been continuously adapted to reflect these changes. Unfortunately, none of these animal models have been unequivocally shown or accepted to fit best the clinical entity. For the sake of brevity, the following models need to be mentioned as the most widely used models. Intrauterine sheep at 0.9 gestation $(\sim 135$ days $)$ is thought to be comparable neurodevelopmentally with the term neonate [66], thus, occlusion of the uterine artery for 3060 min with hypoxic ventilation of the ewe elicits true fetal asphyxiation [120], but 
interestingly only $30 \%$ of surviving animals showed neuronal loss in the hippocampus at 72 hours in this model. On the other hand, 10 min UC occlusion in near term lamb resulted in significant neuronal loss in cortical grey- and subcortical white matter areas at 24 hour survival [121]. Although non-human primates (e.g. Rhesus monkey or Macaca nemestrina) are considered as the best models of the neonate, their widespread use is prohibited because of strict ethical rules. Ranck et al. reported a PA/HIE macaque model induced by detaching the placenta after hysterotomy [63]. Marked neuronal damage in brainstem nuclei, thalamus as well as in the basal ganglia was observed whilst the cortex suffered the least damage. UC occlusion in near-term macaque fetuses resulted in classic signs of HIE after birth (e.g. muscle hypotonia, severely depressed EEG and seizures), moreover, neuropathology evaluation at 4 month of age indicated significant cortical neurodegeneration [122]. Finally, the extensive work of Myers and his colleagues [123, 124] over two decades revealed 4 patterns of newborn brain injury in PA/HIE full-term macaque models, based on the type of the experimental insults (whether anoxia/hypoxemia is developed combined with or without acidemia) [125]. It means, cardiovascular responses of the fetal animals along with the neurohistology findings observed in their brains showed remarkable correlation to brain damage, observed in neonates.

The newborn piglet, as a deputy of the term neonate, is one of the most accepted large animal models in paediatric research since the 1980s. Historically, first quite unsophisticated swine PA models were introduced, focusing on different aspects (e.g. hypoxia, asphyxia or cerebral ischemia) of PA. HI stress was induced for instance by covering the head of the animals with a face mask or eliciting airway obstruction [126, 127], or by increasing the respiratory dead space [128, 129]. Although hypotension and respiratory arrest (ceased gasping) indicated the severity of these insults, still none of these animals manifested the symptoms of HIE during the recovery period. In another, intrauterine swine PA model, 5-8 min UC occlusion resulted only in moderate changes in fetal blood gas and metabolite levels, and subsequently the delivered piglets could drink independently within 24 hours of life. Moreover, the UC occlusion did not influence body weight gain of the newborns during the 72 hour survival period [130]. Isolated cerebral ischemia, induced by raised intracranial pressure elicits HI brain damage in newborn pigs, nevertheless, it does not induce alterations in blood gases and $\mathrm{pH}$ characteristic of PA or unaffects blood biomarkers and systemic blood pressure changes due to the compensatory blood withdrawal [131]. In addition, controlled bleeding-induced hypotension [132] or the ligation of the brachiocephalic artery [82] in pigs 
may also elicit cerebral hypoxia/ischemia but their clinical relevancy is questionable. Historically, bilaterally induced PTX model was employed in our laboratory based on the work of Temesvári et al. [83]. Although the model was able to reproduce all of the critical hallmarks of PA but animal mortality rate was high due to cardiovascular failure as the PTX was not resolved until asystole occurred. Still, no significant neuropathologic alterations were reported at 24 hour of survival [133]. Then, our laboratory tested the effects of suspended ventilation with clamped endotracheal tube in anesthetized, artificially ventilated animals but cerebrocortical electric activity recovered soon after the experimental asphyxia and brain histology analysis revealed only mild neuronal damage at 24 hours [105]. Thus, establishing a $\mathrm{PA} / \mathrm{HIE}$ piglet model reproducing all important features of the clinical entity is challenging, nevertheless, inevitable. The major advantages of our recently introduced PA/HIE piglet model are the followings. First, reproducible, severe and similar degree of mixed (carbonic and lactate) acidosis, hypoxemia along with hypercapnia were observed at the end of experimental asphyxia. Second, none of the animals were lost during asphyxia and immediate reventilation, thus, the animals had stable cardiovascular parameters without pharmacological treatment for hypotension afterwards. Third, brain electric activity remained depressed during the 24-hour survival period in the asphyxiated animals, indicating robust HI brain damage. Fourth, animal mortality rate due to asphyxiation was similar to natural mortality rate observed in piglets at birth. Finally, our model is free of mechanical vessel occlusion and still the brain histology confirmed moderate/severe neuronal damage at 24 hour based on the high incidence of damaged neurons often along with panlaminar necrotic areas in all subcortical and cortical areas, respectively.

Today, ventilation with reduced $\mathrm{FiO}_{2}$ gas mixtures often combined with $\mathrm{BCAO}$ is the most popular method to induce experimental PA. However, the effects of BCAO on cerebral blood flow are still undetermined as the cerebrovascular architecture of the pig has some unique features that are profoundly different from the human anatomy. In the pig, the circle of Willis arises from a complex vascular network, the so-called rete mirabile [134]. The intracranial part of the rete is embedded in the cavernous sinus around the oval foramen of the skull from which the reconstituted internal carotid artery joins the circle. This rete represents a dense network of small arterial arborisation receiving afferent branches in addition to the internal carotid artery also from the termination of the ascending pharyngeal artery, and from other extra- and intracranial arteries: e.g. the ramus anastomicus as well as the arteria anastomica of the external ophtalmic artery [135], offering rich vascular communication 
along both sides of the porcine skull [136]. Furthermore, the bilateral retia are also interconnected by each other on the posterior wall of the sella turcica. The complexity of such vascular network made the swine rete mirabile of the model of human vascular malformations [137, 138]. Accordingly, radionuclide-labeled microsphere measurements revealed unchanged cerebral blood flow in both hemispheres after unilateral carotid artery occlusion in the newborn pig, in addition, under asphyxic conditions as well [107]. Moreover, Haaland et al. [139] along with Burbridge et al. [140] reported swine angiographic studies visualizing the well-developed anastomosis system between the intra- and extracranial arteries, suggesting the inability of inducing focal cerebral ischemia by single extracerebral artery ligation in pigs. To the best of our knowledge, our current results reported the effects of different ventilation conditions with BCAO on the cortical microcirculation in newborn pigs for the first time. Our LSCI/LASCA measurements revealed unchanged cerebrocortical blood flow in response to BCAO under normoxic or hypoxic ventilation or even under asphyxic (clamped endotracheal tube) conditions without affecting pial arteriole diameters. Consequently, we speculate that effective PA induction in this species does not necessarily need to involve BCAO, and BCAO likely does not affect PA severity in a significant matter. Still, mechanical vessel occlusion is still being employed despite the controversies concerning its usefulness.

Our current PA/HIE experimental design have been based on pilot studies using different asphyxia protocols in which EEG activity was selected as a primary indicator of HIE severity for the following reasons. First, Helmy et al. presented a PA/HIE newborn rat model, asphyxiating spontaneously breathing animals in $6 \% \mathrm{O}_{2}$ mixed with $9 \% \mathrm{CO}_{2}$ (and balance $\mathrm{N}_{2}$ ) for 60 minutes [141]. Interestingly, the hypoxic-hypercapnic stress elicited progressive cerebral acidosis that shifted to alkalosis upon room air reventilation, increasing the incidence of neonatal seizures. To reproduce the same conditions in a large PA/HIE model as well, the similar gas mixture was given to newborn pigs, however, in spite of the gradual increase in the hypoxic-hypercapnic duration (30-40-60 minutes, 2-2-2 animals), the piglets suffered only mild asphyxic damage, indicated by the quick (within 4 hours) restoration of the EEG amplitudes. Next, the $\mathrm{FiO}_{2}$ was further lowered to $6 \%$ without changing the percentage of $\mathrm{CO}_{2}$ to elicit greater cerebral injury. Thus, piglets were asphyxiated for 35, 25 and 15 minutes (2-2-3 animals, respectively) duration. Brain electric activity remained isoelectric/depressed at 4 hour of survival, nevertheless, severe cardiovascular failure occurred in most animals after 35 and 25 minutes of stress and these animals could maintain their MABP depending on continuous inotropic therapy during the observation period ( 24 hours). On the other hand, in 
the 15-minute asphyxiation group, EEG activity was quickly restored as well. Consequently, we chose the 20-minute asphyxia with $6 \% \mathrm{O}_{2}-20 \% \mathrm{CO}_{2}$ and reduced $\mathrm{RR}$ that resulted in reproducible, severe but still reversible brain damage in most animals. Interestingly, blood gas and $\mathrm{pH}$ values at the last minute of asphyxia, indicated almost similar values after the different asphyxia protocols, hence, we speculate that not only the severity but the duration of hypoxemia, hypercapnia along with acidosis altogether influence the severity of perinatal brain damage. In our experiments, cEEG recording was proved to be a good guide to titrate the severity of hypoxic-hypercapnic ventilation, moreover, to induce reproducible and reversible brain damage in piglets.

cEEG monitoring offers a non-invasive, real-time recording of cerebrocortical electric activity at bedside. In addition, the ability of the Apgar scores with the umbilical blood gas values is weak to predict safely the severity and outcome of HIE [23], cEEG is thought to be an important method to identify early and accurately sick babies who could benefit from neuroprotective interventions. The prognostic value of cEEG is well-known since 1972 when Monod et al. reported a statistical, retrospective EEG study on 270 asphyxiated children proclaiming that normal neonatal EEG has favorable prognostic significance for good outcome in childhood [142]. Since then, numerous clinical studies could confirm the good predictive value of early (during the first 24-72 hours of life) cEEG recording, therefore, assessing brain electric activity is a routinely applied method in the field of neonatology. Nevertheless, the interpretation of neonatal electric patterns requires trained neurophysicians because of many reasons. First, cortical electric activity varies progressively with postnatal development, second, there is no clear consensus how to grade EEG patterns in neonates with HIE and in addition, some anticonvulsants interfere the EEG waves and suppress brain electric activity sometimes entirely. Thus, to quantify EEG patterns, numerous scoring systems were introduced, principally based on the amplitude of the waves, the continuity, the hemispheric asymmetry, the presence or absence of sleep-wake cycles, seizures along with special EEG features [30]. In a clinical study of Pezzani et al., neonatal EEG recorded during the first 24 hours of life provided valuable information to assess early neonatal brain damage in full-term newborns and to select survivors of PA for intensive care [143]. Interestingly, normal and mildly abnormal EEG traces in full-term asphyxiated neonates, recorded as early as 6 hours of life, predicted reliably normal neurodevelopmental outcome at 2 years, however, as electric patterns changed with increasing survival time, the greatest predictive value of EEG was calculated at 48 hours of survival [31]. Wertheim et al. introduced a computer 
analysis method to evaluate the continuity of brain electric activity within 24 hours of life in children, manifesting symptoms of HIE after birth asphyxiation and confirmed that continuous background activity correlates well with good outcome whilst maximal EEG suppression during this observation period was associated with poor prognosis [144]. Finally, Pressler et al. found that if EEG activity in full-term asphyxiated babies remains abnormal for more that 8-12 hours on the first day of life predicts poor prognosis [145]. However, we could not perform aEEG recordings in our experiments, but we admit that it represents one of the most commonly used non-invasive monitoring devices of critically sick neonates [146].

Successful resuscitation/reventilation will restore $\mathrm{O}_{2}$ /glucose delivery along with reactive increases in cerebral blood flow in asphyxiated animals, although the early reventilation/re-oxygenation period further aggravates the primary neonatal brain injury because of the activation of various enzymes (e.g. XO, COX-2. NOS) in the brain contributing to the excessive generation of free radicals related oxidative stress [147]. High $\mathrm{FiO}_{2}$ during resuscitation is a proven contributor to brain injury as in both our [148] and other laboratories [149, 150], resuscitation with $\mathrm{FiO} 2: 1.00$ after experimental asphyxia negatively affected cerebral neuropathology in large animal models of PA. Therefore, beside adequate neonatal resuscitation recommended by the current guidelines [112, 151], the subsequent "latent phase" between the resolution of the primary and onset of the secondary energy failure offers a potential time window for the initiation of neuroprotective interventions to alleviate reperfusion-induced neuronal injury. Incipiently, numerous PA/HIE animal models (explored in human term equivalent age of rats, sheep and pig) showed that $\mathrm{TH}$, initiated shortly ( 6 hours) after the onset of resuscitation can ameliorate HI brain damage [152-154]. Based on the ample preclinical evidence, large, multi-centre clinical studies (the TOBY [155], the CoolCap [156] and the NICHD trials [157]) were recruited with strict criteria to assess the beneficial effects of controlled body cooling on neonates diagnosed with HIE. Fortunately, the results obtained from these trials declared better neurodevelopmental outcome in neonates receiving TH treatment. Consequently, the controlled mild whole body cooling became the gold standard care of survivors after moderate/severe PA [158]. It is also noteworthy, that some PA/HIE newborn piglet models also contributed remarkably to the exploration of the feasibility of body cooling $[159,160]$ and merging these preclinical and clinical observations, guidelines were proposed for the entry criteria for hypothermic treatment, in addition, for the optimization of the onset, length as well as the depth of cooling [161, 162]. Currently, TH is the only clinically proven neuroprotective intervention for HIE, however, the exact molecular 
mechanisms of the beneficial effects are not fully discovered yet. Despite the significant neuroprotection, $\mathrm{TH}$ alone is not able to ameliorate completely the HI-induced cerebral damage, and in addition, not all asphyxiated babies profit from total body cooling. Thus, synergistic therapies are sought to augment the beneficial effects of cooling.

In 2007, Ohsawa et al. [100] reported the antioxidant properties of molecular $\mathrm{H}_{2}$ in Nature Medicine Journal and since then numerous gases were experimentally tested after cerebral hypoxia/ischemia for putative neuroprotective purposes, e.g. the noble gases argon [163-165], helium [163] or xenon [90], with or without the combination of TH. Resuscitation with a gas mixture containing xenon was also performed in term asphyxiated neonates and Dingley et al. reported the feasibility of the noble gas administration in clinical settings [166]. Despite adequate neuroprotection, the availability and costs of these gases can limit their routine use at bedside. Interestingly, a recent open-label, randomised clinical trial of xenon administration could not demonstrate improved neuroprotection with the combination of $\mathrm{TH}$ in survivors of PA [167].

$\mathrm{H}_{2}$ is a colorless, odourless and nontoxic gas, that can be abundantly produced from water hydrolysis [168] or chemical reactions [101, 102] and thus easily affordable and available. Although $\mathrm{H}_{2}$ is known to be flammable in air, it cannot combust below $4 \%$ and the optimal neuroprotective concentration around $2 \%$ could be safely administered even in a hospital setting. The major advantages of the gas are the small molecular diameter along with the amphiphilic solubility, thus, diatomic $\mathrm{H}_{2}$ can be dissolved in aqueous solutions and be able to penetrate biological membranes as well. This is an important physical characteristic of a putative antioxidant as hydrophilic substances cannot/moderately pass through the biomembranes (in the presence of specific transport proteins) whilst hydrophobic ones remain dissolved in lipid layers, limiting their antioxidant capacity. It is noteworthy, that molecular $\mathrm{H}_{2}$ is rapidly distributed in subcellular compartments, e.g. in cell nucleus or mitochondria, more importantly, is able to penetrate easily the blood-brain-barrier as well. Interestingly, colon bacteria are also able to produce quantities of molecular $\mathrm{H}_{2}$ via anaerobic fermentation [169], however, it is consumed by other bacteria immediately or exhaled through the lungs rapidly. Nevertheless, intestinal $\mathrm{H}_{2}$ production shows correlation to the alimentary status of the animal or the human as plasma levels of molecular $\mathrm{H}_{2}$ are not detectable during fasting but during/after feeding [170]. Oral administration of lactulose can dramatically induce bacterial fermentation and contribute to endogenous $\mathrm{H}_{2}$ production as well [171]. Thus, the dependence on the alimentary status along with the quick elimination, endogenous $\mathrm{H}_{2}$ per se may not offer 
strong neuroprotection against reperfusion-induced oxidative damage, consequently, exogenous supplementation of the gas is required for possible antioxidant purposes even in adult patients.

Therapeutic $\mathrm{H}_{2}$ can be safely administered via inhalation, drinking or injection/infusion of $\mathrm{H}_{2}$-rich saline solution. The advantage of $\mathrm{H}_{2}$ inhalation is the rapid distribution of the gas in blood plasma, however, continuous gas administration requires special respiratory circuitry. On the other hand, drinking $\mathrm{H}_{2}$-rich water is cheaper and can be easily produced (e.g. immersing magnesium bar into water liberates molecular $\mathrm{H}_{2}$ ). Though, $\mathrm{H}_{2}$ penetrates glass and plastic bottles easily, consequently, steady concentration of the gas cannot be preserved for long period. Infusion of $\mathrm{H}_{2}$-rich saline solution was proved to be safe in patients after cerebral ischemic stroke [172], although extra fluid load in several cases may risk cardiovascular instability. After administration, molecular $\mathrm{H}_{2}$ can be detected in tissues. Ono et al. reported that 30 minute inhalation of a gas mixture containing 3-4\% $\mathrm{H}_{2}$ in air raises blood plasma $\mathrm{H}_{2}$ levels dose-dependently to $10-20 \mu \mathrm{mol} / 1$, respectively, without jeopardising physiologic parameters or blood metabolite levels in patients [173]. In addition, blood plasma $\mathrm{H}_{2}$ concentration shows arteriovenous difference, measured by gas chromatography, indicating the incorporation of the gas into tissues [100]. Finally, animal experiments allow the measurement of tissue level of $\mathrm{H}_{2}$ gas by specific $\mathrm{H}_{2}$ electrodes [174].

Unfortunately, the exact molecular target of the diatomic $\mathrm{H}_{2}$ is still not revealed, although research studies over the past 20 years proposed a large amount of molecular mechanisms observed during $\mathrm{H}_{2}$ administration. In the seminal work of Ohsawa et al. [100], cell free experiments showed that molecular $\mathrm{H}_{2}$ selectively decreases the levels of $\mathrm{OH}^{*}$ and $\mathrm{ONOO}^{\circ}$, whilst the levels of other reactive species (e.g. hydrogen peroxide, superoxide anion $\left[{ }^{\circ} \mathrm{O}_{2}\right]$ or nitric oxide $\left.\left[\mathrm{NO}^{\bullet}\right]\right)$ remained unchained [100]. The selective ability of the gas to neutralize principally the $\mathrm{OH}^{*}$ is supposed by the strongest oxidative strength of the radical. Due to these advantageous physicochemical properties of $\mathrm{H}_{2}$, numerous research groups tested molecular $\mathrm{H}_{2}$ in various $\mathrm{HI} /$ reperfusion animal models, focusing on its anti-oxidant, anti-inflammatory and anti-apoptotic effects, consequently, $\mathrm{H}_{2}$ was introduced to almost every field of research where oxidative stress plays an important role in cellular damage [102, 175177]. For the sake of brevity, in a myocardial ischemia rat model $2 \% \mathrm{H}_{2}$ inhalation significantly improved left ventricular contraction meanwhile reduced the infarct size and the level of oxidative injury, assessed by $8-\mathrm{OHdG}$ immunohistochemistry staining [174]. In addition, $\mathrm{H}_{2}$-rich saline markedly ameliorated the renal functions after experimental ischemia 
in a rat model, moreover, it decreased the level of the pro-inflammatory cytokines (e.g. tumor necrosis factor alpha) and alleviated oxidative stress by decreasing the tissue levels of malondialdehyde (MDA) and 8-OHdG [178]. In an other model of retinal ischemia/reperfusion injury, daily $\mathrm{H}_{2}$ inhalation for a week significantly preserved the functions of retinal ganglion cells in rats by inhibiting the overexpression of inflammatory cytokines [179]. Furthermore, molecular $\mathrm{H}_{2}$ was found to be neuroprotective in various middle cerebral artery occlusion (MCAO) rat models as well as $\mathrm{H}_{2}$ administration markedly decreased the infarct size [180], attenuated oxidative injury indicated by the decreased levels of MDA immunostaining [181], decreased caspase-3 staining along with the number of 8OHdG positive cells in the infarct area $[168,171,182]$ and finally, increased the activities of anti-oxidant enzymes (e.g. superoxide dismutase and catalase) [103]. In transient global cerebral ischemia (TGCI) rat models, $\mathrm{H}_{2}$ combatted effectively against I/R injury as well [183] and improved the cognitive functions of the animals via preserving neurons in the hippocampal CA1 region [184]. In addition, our laboratory could also prove the protective effects of the gas after TGCI in rats as $2.1 \% \mathrm{H}_{2}$ resuscitation decreased the ischemia-induced expression of COX-2 and connexin proteins in the parietal cortex and the hippocampus [185]. To the best of our knowledge, our laboratory was the first who confirmed the neuroprotective effects of $2.1 \%$ molecular $\mathrm{H}_{2}$ resuscitation in a PA (suspended artificial ventilation for 10 min) large animal model [186], indicated by the preserved pial arteriolar vascular reactivity to hypercapnic ventilation at 4 hour of survival. Moreover, 4 hour resuscitation with the similar gas mixture afforded also neuroprotection in our previous, subacute swine model (24 hour survival) of PA (clamped endotracheal tube with suspended ventilation) [105]. Interestingly, some but not all experimental studies could report beneficial effects from $\mathrm{H}_{2}$ resuscitation in the field of perinatology reporting conflicting data. For instance, although Cai et al. reported that asphyxiated rat pups, receiving intraperitoneal injection of $\mathrm{H}_{2}$-rich saline, performed better neurologic outcome at 5 weeks compared to asphyxia control animals [187], nevertheless Matchett $e t$ al. found that survivors of moderate or severe experimental asphyxia did not profit from $\mathrm{H}_{2}$ administration, and in addition, $\mathrm{H}_{2}$ therapy preceding the insult was associated with worse neurologic outcome [188].

Beside the putative free radical scavenging capacity, animal studies and our experiments as well indicated safe and well-tolerable administration of molecular $\mathrm{H}_{2}$. Moreover, no adverse effects of $\mathrm{H}_{2}$ treatment were documented yet enabling clinical researchers to introduce the gas into human health care, principally in elderly patients in 
Japanese hospitals. For instance, in a 6 month trial of hemodialysis, patients receiving $\mathrm{H}_{2}$-rich dialysis solution showed lower levels of systemic inflammatory markers along with improved blood pressure control [189]. Kajiyama et al. observed improved glucose tolerance in a randomized, double-blind, placebo-controlled study, in which $\mathrm{H}_{2}$ saline solution was administered to patients for 8 weeks, manifesting symptoms of diabetes mellitus type 2 [190]. In a study of Ono et al., $\mathrm{H}_{2}$ enriched saline solution could potentiate the anti-oxidant effects of Edaravone treatment, evaluated by MRI in patients with brainstem infarction [191]. Since the initial clinical successes with molecular $\mathrm{H}_{2}$, recent promising results were also obtained in Parkinson's disease [192], chronic hepatitis [193], rheumatoid arthritis [194] as well as in mitochondrial myopathies [195]. A recently founded non-profit organization, Molecular Hydrogen Foundation, aimed to promote molecular $\mathrm{H}_{2}$ research in clinical studies along with providing a comprehensive resource medical organizations (http://www.molecularhydrogenfoundation.org/).

Beside the reperfusion-induced oxidative damage, alterations in $\mathrm{pH}_{\text {brain }}$ can also significantly affect neuronal injury. Regulation of $\mathrm{pH}$ in the brain is finely tuned under physiologic conditions [196], thus, perturbations in extracellular $\mathrm{pH}$ would highly influence neuronal excitability. In vitro studies reported that acidosis suppresses the activity of the Nmethyl-D-aspartate (NMDA) ion channel meanwhile alkalosis facilitates ion currents through the NMDA receptors in rat hippocampal neurons [197]. Moreover, environmental $\mathrm{pH}$ changes similarly modulate the conductance and gating properties of voltage-gated $\mathrm{Na}^{+}$and $\mathrm{Ca}^{++}$ion channels in rat hippocampal cells [198]. Thus, both cerebral acidosis and alkalosis can significantly contribute to neuronal dysfunctions. For instance, Helmy et al. [141] reported that asphyxia, induced by hypoxic-hypercapnic $\left(\begin{array}{llll}9 \% & \mathrm{O}_{2}-20 \% & \mathrm{CO}_{2}\end{array}\right)$ gas mixture in spontaneously breathing rat pups, elicited cerebral acidosis during asphyxia and interestingly, during reventilation, the $\mathrm{pH}_{\text {brain }}$ progressively shifted towards alkalosis that was associated with the incidence of increasing seizure burden in the pups. Because of its importance to determine neuronal injury, numerous in vivo studies recorded $\mathrm{pH}_{\text {brain }}$ changes in various $\mathrm{HI}$ animal models. However, quantitative data on $\mathrm{pH}_{\text {brain }}$ changes during/after PA in large animal models are very scarce in the literature, thus, our current observations provide important new information to the pathophysiology of HIE in piglets. We are aware of only one previous study [199] that assessed $\mathrm{pH}_{\text {brain }}$ with similar technique after PA in piglets. In this study, Bender et al. induced 30 min PA with a hypoxic-hypercapnic gas mixture $\left(5-8 \% \mathrm{O}_{2}-7 \% \mathrm{CO}_{2}\right)$ [199], at the end of PA the reported $\mathrm{pH}_{\text {brain }}$ was $6.26 \pm 0.14$, almost half $\mathrm{pH}$ unit higher than in 
our study likely at least in part due to the much lower $\mathrm{P}_{\mathrm{a}} \mathrm{CO}_{2}$ values $(61 \pm 1$ vs. $160 \pm 23 \mathrm{mmHg}$, [199] $v s$. present study, respectively). In addition, the previous study did not follow up on $\mathrm{pH}_{\text {brain }}$ changes beyond 4-hour survival, in contrast, our current measurements extended the observation period considerably, to 24 hours of survival. According to our present data, no major secondary alterations in $\mathrm{pH}_{\text {brain }}$ levels are present in the piglet up to 24 hours despite obvious development of neuronal damage. Our results suggest that $\mathrm{pH}_{\text {brain }}$ alterations play an important role in the pathophysiology of HIE development as very severe acidosis during the acute phase of asphyxia, and possibly brain alkalosis after the first 24-hour of reventilation. This suggestion is corroborated by observations in human neonates. MRS is a promising method to measure and to follow mainly intracellular $\mathrm{pH}$ changes in the brain [200]. Using MRS, Hope et al. reported no significant changes in brain $\mathrm{pH}(7.13 \pm 0.05)$ from term asphyxiated newborns during the first day of life that essentially match our experimental results in the present study, Importantly, brain $\mathrm{pH}$ rose during the following days indicating the belated development of brain alkalosis [201]. Robertson et al. reported also brain alkalosis appearing in term neonates in the first 2 weeks of life after PA. The extent of neonatal brain injury showed good correlation with the elevation of cerebral $\mathrm{pH}$ that persisted for months afterwards corresponding to poor neurodevelopmental outcome [202]. Interestingly, Chopp et al. reported in adult rats that forebrain ischemia induced by transient BCAO and arterial hypotension also triggered post-ischemic cerebral alkalosis appearing first only after 24 hour of survival and was still present at 48 hours [203]. Dynamics of $\mathrm{pH}_{\text {brain }}$ alterations in response to HI stress can thus clearly show species, and age specific variations, the large animal piglet HIE model may follow a more similar course to the human, and may be markedly different from the rat.

There are some limitations of the findings presented in the dissertation. The 24-hour survival period may have not allowed us to assess fully the long-term neuroprotective efficacy of molecular $\mathrm{H}_{2}$, and we were not able in this period to test the effect of $\mathrm{H}_{2}$ with $\mathrm{TH}$, the current gold standard care of HIE patients. The time frame was simply not enough to initiate $\mathrm{TH}$, as rewarming alone from hypothermia would have lasted for approximately 10 hours $\left(0.5^{\circ} \mathrm{C} /\right.$ hour $\left.[161,162]\right)$, allowing only a very short period at the therapeutic core temperature. Furthermore, literature data also suggested against TH in this time frame, as Thoresen et al. reported no beneficial effects after 24 -hour mild $\mathrm{TH}$ (rectal temperature: $35^{\circ} \mathrm{C}$ ) in a $\mathrm{PA} / \mathrm{HIE}$ piglet model. However, the failure to show beneficial effects might have been caused by the hypothermia-induced stress and shivering in the unsedated animals [204]. In contrast, short- 
term TH can have neuroprotection. In another study, 7-day-old rat pups were subjected to experimental PA and only 6-hour-long postasphyxial hypothermia (rectal temperature: $32^{\circ} \mathrm{C}$ ), and this limited TH was found to be remarkably neuroprotective at 6 week survival [205]. The equivocal reports of short-term $\mathrm{TH}$ in the literature could be explained by the differences in animal species, the type of anesthesia and experimental protocol, nevertheless, the onset, duration and deepness of cooling also influence the outcome. Increasing the survival period (up to 48-72 hours) and testing whether $\mathrm{TH}$ potentiates the neuroprotective effects of molecular $\mathrm{H}_{2}$ remains our future aim. Next, the type of anesthesia may influence neuronal functions and is often criticized in the literature. Ethical committees understandably require adequate anesthesia/analgesia to reduce/eliminate pain/discomfort in the laboratory animals. We tried to select anesthetic drugs that retain as much as possible the translational strength of the piglet model as human HIE patients are of course not generally anesthetized. Morphine with midazolam are frequently administered analgesic regimens in neonatal intensive care units [206]. Morphine was proved to alleviate effectively ventilation-induced pain and stress in neonates [207]. Furthermore, midazolam provides stable sedation in artificially ventilated babies, moreover, is routinely given to treat neonatal seizures, it was shown to unaffect brain electric activity, thereby not jeopardising amplitude-based EEG analysis [208]. In summary, despite these limitations, we believe that our studies can contribute to the better understanding of HIE pathophysiology and provide a good model for testing neuroprotective treatments. 


\section{CONCLUSION}

Our research effort focused on assessing the putative neuroprotective effect of molecular $\mathrm{H}_{2}$ in a translational model of moderate/severe HIE. To reach this goal, we first critically examined the methodology and the translational potential of previously published piglet PA/HIE models. During this process, we demonstrated that BCAO in the newborn piglet does not affect the cerebrocortical microcirculation e.g. it does not induce significant ischemia under various ventilation conditions. Thus, the usefulness of mechanical vessel occlusion to augment the severity of PA insult has been questioned. We then successfully designed a subacute HIE model, where anesthesia/analgesia and the methodology to induce PA have been chosen to maximize the translational potential of the model. Our new PA/HIE model has been painstakingly characterized: changes in metabolism, hemodynamic parameters, brain electrical activity and uniquely also $\mathrm{pH}_{\text {brain }}$ were determined, and demonstrated to comply with the data obtained from neonates. Our $\mathrm{pH}_{\text {brain }}$ measurements yielded that acidosis during PA may be 10 times ( $1 \mathrm{pH}$ unit) higher compared to the arterial blood. Neuropathology revealed that our new model was capable inducing severe and reproducible structural brain damage in accordance with the suppression of brain electric activity. Using this established PA/HIE model we demonstrated that reventilation with $2.1 \%$ molecular $\mathrm{H}_{2}$ facilitated the recovery of EEG amplitudes and ameliorated neuronal damage virtually in all examined areas. The mechanism of molecular $\mathrm{H}_{2}$ action moreover appears to include alleviated oxidative stress, indicated by the lower number of 8-OHdG immunopositive nuclei in the assessed regions. Our data suggest a marked neuroprotective potential of post-insult administered molecular $\mathrm{H}_{2}$, therefore we aim to assess the neuroprotective effects of $\mathrm{H}_{2}$ combined with $\mathrm{TH}$ in the future. 


\section{ACKNOWLEDGEMENTS}

First, I would like to say thank to Prof. Gábor Jancsó, the leader of the Theoretical Medicine Doctoral School, who accepted my application to the Doctoral School.

Most of all, it is a genuine pleasure to express my thanks and gratitude to my supervisor and friend, Ferenc Domoki MD, who arose my attention in medical physiology with his excellent knowledge then allowed me to join his research group first, as an undergraduate then a postgraduate student. His keen interest on me, prompt inspirations, enthusiasm and dynamism have enabled me to complete my thesis.

Then, I would like to say thank to Valéria Tóth-Szüki for her technical support.

Last, but not least, many thanks belong to my colleagues, family and friends. 


\section{REFERENCES}

1. Zupan, J. and Aahman, E., Perinatal mortality for the year 2000: estimates developed by WHO, World Health Organization. 2005: Geneva.

2. A/RES/55/2, United Nations Millennium Declaration: resolution adopted by the General Assembly. 55/2., Sept 18, 2000.

3. United Nations, The Millennium Development Goals Report. 2015: New York.

4. Sankar, M.J., Natarajan, C.K., Das, R.R. et al., When do newborns die? A systematic review of timing of overall and cause-specific neonatal deaths in developing countries. Journal of Perinatology, 2016. 36: p. S1-S11.

5. The UN inter-agency group for child mortality estimation. Levels and trends in child mortality: report 2013 in. 2013., UNICEF: New York.

6. Lawn, J.E., Cousens, S. and Zupan, J., 4 million neonatal deaths: When? Where? Why? Lancet, 2005. 365(9462): p. 891-900.

7. KSH, Népmozgalom: 2016, in Statisztikai tükör. 2017.

8. Bax, M. and Nelson, K.B., Birth asphyxia: a statement. Developmental Medicine \& Child Neurology, 1993. 35(11): p. 1022-1024.

9. Dauber, I.M., Krauss, A.N., Symchych, P.S. et al., Renal failure following perinatal anoxia. Journal of Pediatrics, 1976. 88(5): p. 851-855.

10. Antonucci, R., Porcella, A. and Pilloni, M.D., Perinatal asphyxia in the term newborn. Journal of Pediatric and Neonatal Individualized Medicine, 2014. 3(2): p. 1-14.

11. Apgar, V., A proposal for a new method of evaluation of the newborn infant. Current Researches in Anesthesia \& Analgesia, 1953. 32(4): p. 260-267.

12. American College of Obstetrics and Gynecology., Task Force on Neonatal Encephalopathy and Cerebral Palsy., American Academy of Pediatrics. Neonatal Encephalopathy and Cerebral Palsy: Defining the Pathogenesis and Pathophysiology. Edited by Washington, DC, American College of Obstetricians and Gynecologists, 2003.

13. Volpe, J.J., Neonatal encephalopathy: an inadequate term for hypoxic-ischemic encephalopathy. Annals of Neurology, 2012. 72(2): p. 156-166.

14. Nelson, K.B. and Leviton, A., How much of neonatal encephalopathy is due to birth asphyxia. American Journal of Diseases of Children, 1991. 145(11): p. 1325-1331.

15. Ellis, M. and Costello, A., Birth asphyxia, Apgar score and neonatal encephalopathy. Indian Pediatrics, 1997. 34(11): p. 975-978.

16. Rosenbaum, P., Cerebral palsy: what parents and doctors want to know. British Medical Journal, 2003. 326(7396): p. 970-974.

17. Aisen, M.L., Kerkovich, D., Mast, J. et al., Cerebral palsy: clinical care and neurological rehabilitation. Lancet Neurology, 2011. 10(9): p. 844-852.

18. Eunson, P., The long-term health, social, and financial burden of hypoxic-ischaemic encephalopathy. Developmental Medicine and Child Neurology, 2015. 57: p. 48-50.

19. Shevell, M.I., The "Bermuda triangle" of neonatal neurology: cerebral palsy, neonatal encephalopathy, and intrapartum asphyxia. Seminars in Pediatric Neurology, 2004. 11(1): p. 24-30.

20. Vannucci, R.C., Hypoxic-ischemic encephalopathy. American Journal of Perinatology, 2000. 17(3): p. 113-120.

21. Sarnat, H.B. and Sarnat, M.S., Neonatal encephalopathy following fetal distress. A clinical and electroencephalographic study. Archives of Neurology, 1976. 33(10): p. 696-705.

22. Murray, D.M., Ryan, C.A., Boylan, G.B. et al., Prediction of seizures in asphyxiated neonates: Correlation with continuous video-electroencephalographic monitoring. Pediatrics, 2006. 118(1): p. 41-46. 
23. Shevell, M.I., Majnemer, A. and Miller, S.P., Neonatal neurologic prognostication: The asphyxiated term newborn. Pediatric Neurology, 1999. 21(5): p. 776-784.

24. Shen, W., Pan, J.H. and Chen, W.D., Comparison of transcranial ultrasound and cranial MRI in evaluations of brain injuries from neonatal asphyxia. International Journal of Clinical and Experimental Medicine, 2015. 8(10): p. 18319-18326.

25. Barnette, A.R., Horbar, J.D., Soll, R.F. et al., Neuroimaging in the evaluation of neonatal encephalopathy. Pediatrics, 2014. 133(6): p. E1508-E1517.

26. Guo, L.L., Wang, D.H., Bo, G.J. et al., Early identification of hypoxic-ischemic encephalopathy by combination of magnetic resonance (MR) imaging and proton $M R$ spectroscopy. Experimental and Therapeutic Medicine, 2016. 12(5): p. 2835-2842.

27. Agut, T., Leon, M., Rebollo, M. et al., Early identification of brain injury in infants with hypoxic ischemic encephalopathy at high risk for severe impairments: accuracy of MRI performed in the first days of life. Bmc Pediatrics, 2014. 14(177): p. 1-7.

28. Kudreviciene, A., Lukosevicius, S., Laurynaitiene, J. et al., Ultrasonography and magnetic resonance imaging of the brain in hypoxic full-term newborns. Medicina-Lithuania, 2013. 49(1): p. 42-49.

29. Sie, L.T.L., van der Knaap, M.S., Oosting, J. et al., MR patterns of hypoxic-ischemic brain damage after prenatal, perinatal or postnatal asphyxia. Neuropediatrics, 2000. 31(3): p. 128136.

30. Walsh, B.H., Murray, D.M. and Boylan, G.B., The use of conventional EEG for the assessment of hypoxic ischaemic encephalopathy in the newborn: A review. Clinical Neurophysiology, 2011. 122(7): p. 1284-1294.

31. Murray, D.M., Boylan, G.B., Ryan, C.A. et al., Early EEG findings in hypoxic-ischemic encephalopathy predict outcomes at 2 years. Pediatrics, 2009. 124(3): p. E459-E467.

32. Maynard, D., Prior, P.F. and Scott, D.F., Device for continuous monitoring of cerebral activity in resuscitated patients. British Medical Journal, 1969. 4(5682): p. 545-546.

33. Tao, J.D. and Mathur, A.M., Using amplitude-integrated EEG in neonatal intensive care. Journal of Perinatology, 2010. 30: p. 73-81.

34. Hagberg, H., Edwards, A.D. and Groenendaal, F., Perinatal brain damage: The term infant. Neurobiology of Disease, 2016. 92: p. 102-112.

35. Fatemi, A., Wilson, M.A. and Johnston, M.V., Hypoxic-ischemic encephalopathy in the term infant. Clinics in Perinatology, 2009. 36(4): p. 835-858.

36. Rainaldi, M.A. and Perlman, J.M., Pathophysiology of birth asphyxia. Clinics in Perinatology, 2016. 43(3): p. 409-422.

37. Brillault, J., Lam, T.I., Rutkowsky, J.M. et al., Hypoxia effects on cell volume and ion uptake of cerebral microvascular endothelial cells. American Journal of Physiology-Cell Physiology, 2008. 294(1): p. C88-C96.

38. Silverstein, F.S., Naik, B. and Simpson, J., Hypoxia-ischemia stimulates hippocampal glutamate efflux in perinatal rat brain: an in vivo microdialysis study. Pediatric Research, 1991. 30(6): p. 587-590.

39. Hagberg, H., Mallard, C., Rousset, C.I. et al., Mitochondria: hub of injury responses in the developing brain. Lancet Neurology, 2014. 13(2): p. 217-232.

40. Johnston, M.V., Excitotoxicity in perinatal brain injury. Brain Pathology, 2005. 15(3): p. 234240.

41. Glick, D., Barth, S. and Macleod, K.F., Autophagy: cellular and molecular mechanisms. Journal of Pathology, 2010. 221(1): p. 3-12.

42. Ginet, V., Pittet, M.P., Rummel, C. et al., Dying neurons in thalamus of asphyxiated term newborns and rats are autophagic. Annals of Neurology, 2014. 76(5): p. 695-711.

43. Ginet, V., Puyal, J., Clarke, P.G.H. et al., Enhancement of autophagic flux after neonatal cerebral hypoxia-ischemia and its region-specific relationship to apoptotic mechanisms. American Journal of Pathology, 2009. 175(5): p. 1962-1974. 
44. Sanderson, T.H., Reynolds, C.A., Kumar, R. et al., Molecular mechanisms of ischemiareperfusion injury in brain: pivotal role of the mitochondrial membrane potential in reactive oxygen species generation. Molecular Neurobiology, 2013. 47(1): p. 9-23.

45. Olah, O., Nemeth, I., Toth-Szuki, V. et al., Regional differences in the neuronal expression of cyclooxygenase-2 (COX-2) in the newborn pig brain. Acta Histochem Cytochem, 2012. 45(3): p. 187-192.

46. Bhalala, U., Al Hazmi, A., Kulikowicz, E. et al., Sex Influences Microglial Morphology at Baseline and after Hypoxic Ischemic Injury in Immature Brain. Critical Care Medicine, 2015. 43(12).

47. Hagberg, H., Mallard, C., Ferriero, D.M. et al., The role of inflammation in perinatal brain injury. Nature Reviews Neurology, 2015. 11(4): p. 192-208.

48. Cady, E.B., Iwata, O., Bainbridge, A. et al., Phosphorus magnetic resonance spectroscopy $2 \mathrm{~h}$ after perinatal cerebral hypoxia-ischemia prognosticates outcome in the newborn piglet. Journal of Neurochemistry, 2008. 107(4): p. 1027-1035.

49. Shankaran, S., The postnatal management of the asphyxiated term infant. Clinics in Perinatology, 2002. 29(4): p. 675-692.

50. Wyatt, J.S., Edwards, A.D., Azzopardi, D. et al., Magnetic resonance and near infrared spectroscopy for investigation of perinatal hypoxic-ischaemic brain injury. Archives of Disease in Childhood, 1989. 64(7 Spec No): p. 953-963.

51. Hassell, K.J., Ezzati, M., Alonso-Alconada, D. et al., New horizons for newborn brain protection: enhancing endogenous neuroprotection. Archives of Disease in Childhood-Fetal and Neonatal Edition, 2015. 100(6): p. F541-F551.

52. Robertson, N.J., Cox, I.J., Cowan, F.M. et al., Cerebral intracellular lactic alkalosis persisting months after neonatal encephalopathy measured by magnetic resonance spectroscopy. Pediatric Research, 1999. 46(3): p. 287-296.

53. Serpero, L.D., Bellissima, V., Colivicchi, M. et al., Next generation biomarkers for brain injury. Journal of Maternal-Fetal \& Neonatal Medicine, 2013. 26: p. 44-49.

54. Chalak, L.F., Sanchez, P.J., Adams-Huet, B. et al., Biomarkers for severity of neonatal hypoxicischemic encephalopathy and outcomes in newborns receiving hypothermia therapy. Journal of Pediatrics, 2014. 164(3): p. 468-474.

55. Thornberg, E., Thiringer, K., Hagberg, H. et al., Neuron-specific enolase in asphyxiated newborns - association with encephalopathy and cerebral function monitor trace. Archives of Disease in Childhood, 1995. 72(1): p. F39-F42.

56. Paliwal, M., Paliwal, P., Varma, M. et al., Study of neuron specific enolase (NSE) in perinatal asphyxia \& its role as an early marker of brain injury. Journal of Evidence Based Medicine and Healthcare, 2016. 3(67): p. 3640-3643.

57. Verdú Pérez, A., Falero, M., Arroyos, A. et al., Blood neuronal specific enolase in newborns with perinatal asphyxia. Revista de Neurologia, 2001. 32(8): p. 714-717.

58. LeGallois, M., Experiments on the Principle of Life. 1813, Philadelphia, PA.

59. Bert, P., Lecons sur La Comparée de la Respiration. 1870: Paris.

60. Bauer, D.J., The effect of asphyxia upon the heart rate of rabbits at different ages. The Journal of Physiology, 1938. 93(2): p. 90-103.

61. Bauer, D.J., Vagal reflexes appearing in asphyxia in rabbits at different ages. The Journal of Physiology, 1939. 95(1): p. 187-202.

62. Windle, W.F., Becker, R.F. and Weil, A., Alterations in brain structure after asphyxiation at birth: an experimental study in the guinea pig. Journal of Neuropathology Experimental Neurology, 1944. 3(3): p. 224-238.

63. Ranck, J.B. and Windle, W.F., Brain damage in the monkey, macaca mulatta, by asphyxia neonatorum. Experimental Neurology, 1959. 1(2): p. 130-154.

64. Levine, S., Anoxic-ischemic encephalopathy in rats. American Journal of Pathology, 1960. 36: p. 1-17. 
65. Rice, J.E., 3rd, Vannucci, R.C. and Brierley, J.B., The influence of immaturity on hypoxicischemic brain damage in the rat. Annals of Neurology, 1981. 9(2): p. 131-141.

66. Dobbing, J. and Sands, J., Comparative aspects of the brain growth spurt. Early Human Development, 1979. 3(1): p. 79-83.

67. Bustad, L.K. and McClellan, R.O., Swine in biomedical research. Science, 1966. 152(3728): p. 1526-1530.

68. Bustad, L.K., Pigs in the laboratory. Scientific American, 1966. 214(6): p. 94-100.

69. Altman, P.L. and Dittmer, P.S., Growth. 1962, Washington, D.C.: Federation of American Societies for Experimental Biology.

70. Dickerson, J.W. and Dobbing, J., Prenatal and postnatal growth and development of the central nervous system of the pig. Proceedings of the Royal Society of London B: Biological Sciences, 1967. 166(1005): p. 384-395.

71. Pond, W.G., Boleman, S.L., Fiorotto, M.L. et al., Perinatal ontogeny of brain growth in the domestic pig. Proc Soc Exp Biol Med, 2000. 223(1): p. 102-108.

72. Dobbing, J. and Sands, J., Quantitative growth and development of human brain. Arch Dis Child, 1973. 48(10): p. 757-767.

73. Dobbing, J., The later development of the brain and its vulnerability. In Scientific Foundations of Paediatrics, ed. J.A. Davis and J. Dobbing. 1974, London: William Heinemann Medical Books. 565-577.

74. English, P.R. and Wilkinson, V., Management of the sow and litter in late pregnancy and lactation in relation to piglet survival and growth. Control of Pig Reproduction, ed. D.J.A.C.a.G.R. Foxcroft. 1982, Butterworths, London, UK.

75. Randall, G.C., The relationship of arterial blood pH and pCO2 to the viability of the newborn piglet. Canadian Journal of Comparative Medicine, 1971. 35(2): p. 141-146.

76. Randall, G.C., Observations on parturition in the sow. I. Factors associated with the delivery of the piglets and their subsequent behaviour. Veterinary Recearch, 1972. 90(7): p. 178-182.

77. Randall, G.C., $p H$ values and blood-gas tensions in the normal piglet during the first 48 hours of life. Biology of the Neonate, 1972. 20(1): p. 68-73.

78. Randall, G.C.B., Form and development of the umbilical-cord in pigs and their association with delivery of viable pigs. American Journal of Veterinary Research, 1989. 50(9): p. 1512-1515.

79. Herpin, P., LeDividich, J., Hulin, J.C. et al., Effect of the level of asphyxia during delivery on viability at birth and early postnatal vitality of newborn pigs. Journal of Animal Science, 1996. 74(9): p. 2067-2075.

80. Goodwin, T.M., Belai, I., Hernandez, P. et al., Asphyxial complications in the term newborn with severe umbilical acidemia. American Journal of Obstetrics \& Gynecology, 1992. 167(6): p. 1506-1512.

81. Leffler, C.W., Busija, D.W., Beasley, D.G. et al., Postischemic cerebral microvascular responses to norepinephrine and hypotension in newborn pigs. Stroke, 1989. 20(4): p. 541-546.

82. Kim, Y.B., Gidday, J.M., Gonzales, E.R. et al., Effect of hypoglycemia on postischemic cortical blood flow, hypercapnic reactivity, and interstitial adenosine concentration. Journal of Neurosurgery, 1994. 81(6): p. 877-884.

83. Temesvari, P., Hencz, P., Joo, F. et al., Modulation of the blood-brain-barrier permeability in neonatal cyto-toxic brain edema - laboratory and morphological findings obtained on newborn piglets with experimental pneumothorax. Biology of the Neonate, 1984. 46(4): p. 198-208.

84. Martin, L.J., Brambrink, A., Koehler, R.C. et al., Primary sensory and forebrain motor systems in the newborn brain are preferentially damaged by hypoxia-ischemia. Journal of Comparative Neurology, 1997. 377(2): p. 262-285.

85. Lorek, A., Takei, Y., Cady, E.B. et al., Delayed ("secondary") cerebral energy failure after acute hypoxia-ischemia in the newborn piglet: continuous 48-hour studies by phosphorus magnetic resonance spectroscopy. Pediatric Research, 1994. 36(6): p. 699-706. 
86. Poulsen, J.P., Oyasaeter, S., Sanderud, J. et al., Hypoxanthine, xanthine, and uric acid concentrations in the cerebrospinal fluid, plasma, and urine of hypoxemic pigs. Pediatric Research, 1990. 28(5): p. 477-481.

87. Gunn, A.J., Laptook, A.R., Robertson, N.J. et al., Therapeutic hypothermia translates from ancient history in to practice. Pediatric Research, 2017. 81(1-2): p. 202-209.

88. Zanelli, S., Buck, M. and Fairchild, K., Physiologic and pharmacologic considerations for hypothermia therapy in neonates. Journal of Perinatology, 2011. 31(6): p. 377-386.

89. Robertson, N.J., Tan, S., Groenendaal, F. et al., Which neuroprotective agents are ready for bench to bedside translation in the newborn infant? Journal of Pediatrics, 2012. 160(4): p. 544-552.

90. Chakkarapani, E., Dingley, J., Liu, X. et al., Xenon enhances hypothermic neuroprotection in asphyxiated newborn pigs. Annals of Neurology, 2010. 68(3): p. 330-341.

91. Chakkarapani, E., Thoresen, M., Hobbs, C.E. et al., A closed-circuit neonatal xenon delivery system: A technical and practical neuroprotection feasibility study in newborn pigs. Anesthesia and Analgesia, 2009. 109(2): p. 451-460.

92. Van Bel, F., Shadid, M., Moison, R.M.W. et al., Effect of allopurinol on postasphyxial free radical formation, cerebral hemodynamics, and electrical brain activity. Pediatrics, 1998. 101(2): p. 185-193.

93. Chaudhari, T. and McGuire, W., Allopurinol for preventing mortality and morbidity in newborn infants with suspected hypoxic-ischaemic encephalopathy. Cochrane Database of Systematic Reviews, 2008(2): p. 1-22.

94. Jatana, M., Singh, I., Singh, A.K. et al., Combination of systemic hypothermia and $\mathrm{N}$ acetylcysteine attenuates hypoxic-ischemic brain injury in neonatal rats. Pediatric Research, 2006. 59(5): p. 684-689.

95. Heard, K.J., Acety/cysteine for acetaminophen poisoning. New England Journal of Medicine, 2008. 359(3): p. 285-292.

96. Robertson, N.J., Faulkner, S., Fleiss, B. et al., Melatonin augments hypothermic neuroprotection in a perinatal asphyxia model. Brain, 2013. 136(Pt 1): p. 90-105.

97. Tataranno, M.L., Perrone, S., Longini, M. et al., New antioxidant drugs for neonatal brain injury. Oxidative Medicine and Cellular Longevity, 2015.

98. Zhu, C.L., Kang, W.Q., Xu, F.L. et al., Erythropoietin improved neurologic outcomes in newborns with hypoxic-ischemic encephalopathy. Pediatrics, 2009. 124(2): p. E218-E226.

99. Barks, J.D., Liu, Y.Q., Shangguan, Y. et al., Phenobarbital augments hypothermic neuroprotection. Pediatric Research, 2010. 67(5): p. 532-537.

100. Ohsawa, I., Ishikawa, M., Takahashi, K. et al., Hydrogen acts as a therapeutic antioxidant by selectively reducing cytotoxic oxygen radicals. Nature Medicine, 2007. 13(6): p. 688-694.

101. Ohta, S., Molecular hydrogen as a novel antioxidant: overview of the advantages of hydrogen for medical applications. Methods in Enzymology, 2015. 555: p. 289-317.

102. Dixon, B.J., Tang, J. and Zhang, J.H., The evolution of molecular hydrogen: a noteworthy potential therapy with clinical significance. Medical Gas Research, 2013. 3(10): p. 1-12.

103. Li, J.J., Dong, Y.S., Chen, H.G. et al., Protective effects of hydrogen-rich saline in a rat model of permanent focal cerebral ischemia via reducing oxidative stress and inflammatory cytokines. Brain Research, 2012. 1486: p. 103-111.

104. Cai, J., Kang, Z., Liu, W.W. et al., Hydrogen therapy reduces apoptosis in neonatal hypoxiaischemia rat model. Neuroscience Letters, 2008. 441(2): p. 167-172.

105. Olah, O., Toth-Szuki, V., Temesvari, P. et al., Delayed neurovascular dysfunction is alleviated by hydrogen in asphyxiated newborn pigs. Neonatology, 2013. 104(2): p. 79-86.

106. Hall, R.W., Boyle, E. and Young, T., Do ventilated neonates require pain management? Seminars in Perinatology, 2007. 31(5): p. 289-297.

107. Laptook, A.R., Stonestreet, B.S. and Oh, W., The effect of carotid artery ligation on brain blood flow in newborn piglets. Brain Research, 1983. 276(1): p. 51-54. 
108. Busija, D.W., Leffler, C.W. and Wagerle, L.C., Responses of newborn pig pial arteries to sympathetic nervous stimulation and exogenous norepinephrine. Pediatric Research, 1985. 19(11): p. 1210-1214.

109. van Rooij, L.G., van den Broek, M.P., Rademaker, C.M. et al., Clinical management of seizures in newborns : diagnosis and treatment. Paediatric Drugs, 2013. 15(1): p. 9-18.

110. Tsuchida, T.N., Wusthoff, C.J., Shellhaas, R.A. et al., American clinical neurophysiology society standardized EEG terminology and categorization for the description of continuous EEG monitoring in neonates: report of the American Clinical Neurophysiology Society critical care monitoring committee. Journal of Clinical Neurophysiology, 2013. 30(2): p. 161-173.

111. Foster, K.A., Colditz, P.B., Lingwood, B.E. et al., An improved survival model of hypoxia/ischaemia in the piglet suitable for neuroprotection studies. Brain Research, 2001. 919(1): p. 122-131.

112. Sweet, D.G., Carnielli, V., Greisen, G. et al., European Consensus Guidelines on the Management of Respiratory Distress Syndrome - 2016 Update. Neonatology, 2017. 111(2): p. 107-125.

113. UNICEF, WHO, The World Bank, UN-DESA Population division levels and trends in child mortality 2015. 2015: New York, USA.

114. WHO, UNICEF Every Newborn: An Action Plan to End Preventable Deaths. 2014: Geneva.

115. Leviton, A. and Nelson, K.B., Problems with definitions and classifications of newborn encephalopathy. Pediatric Neurology, 1992. 8(2): p. 85-90.

116. Kurinczuk, J.J., White-Koning, M. and Badawi, N., Epidemiology of neonatal encephalopathy and hypoxic-ischaemic encephalopathy. Early Human Development, 2010. 86(6): p. 329-338.

117. Ferriero, D.M., Medical progress - Neonatal brain injury. New England Journal of Medicine, 2004. 351(19): p. 1985-1995.

118. Golubnitschaja, O., Yeghiazaryan, K., Cebioglu, M. et al., Birth asphyxia as the major complication in newborns: moving towards improved individual outcomes by prediction, targeted prevention and tailored medical care. EPMA J., 2011. 2(2): p. 197-210.

119. Kancherla, V., Amendah, D.D., Grosse, S.D. et al., Medical expenditures attributable to cerebral palsy and intellectual disability among Medicaid-enrolled children. Research in Developmental Disabilities, 2012. 33(3): p. 832-840.

120. Gunn, A.J., Parer, J.T., Mallard, E.C. et al., Cerebral histologic and electrocorticographic changes after asphyxia in fetal sheep. Pediatric Research, 1992. 31(5): p. 486-491.

121. Castillo-Melendez, M., Baburamani, A.A., Cabalag, C. et al., Experimental modelling of the consequences of brief late gestation asphyxia on newborn lamb behaviour and brain structure. PLoS One, 2013. 8(11): p. 1-13.

122. Juul, S.E., Aylward, E., Richards, T. et al., Prenatal cord clamping in newborn Macaca nemestrina: a model of perinatal asphyxia. Developmental Neuroscience, 2007. 29(4-5): p. 311-320.

123. Myers, R., The clinical and pathological effects of asphyxiation in the fetal rhesus monkey. Diagnosis and Treatment of Fetal Disorders, 1968: p. 226-249.

124. Myers, R.E., Atrophic cortical sclerosis after asphyxia of newborn monkey. Journal of Neuropathology \& Experimental Neurology, 1968. 27(1): p. 126.

125. Myers, R.E., Four patterns of perinatal brain damage and their conditions of occurrence in primates. Advances in Neurology, 1975. 10: p. 223-234.

126. Herpin, P., Wosiak, F., Le Dividich, J. et al., Effects of acute asphyxia at birth on subsequent heat production capacity in newborn pigs. Research in Veterinary Science, 1999. 66(1): p. 4549.

127. Touloukian, R.J., Posch, J.N. and Spencer, R., The pathogenesis of ischemic gastroenterocolitis of the neonate: selective gut mucosal ischemia in asphyxiated neonatal piglets. Journal of Pediatric Surgery, 1972. 7(2): p. 194-205.

128. Alward, C.T., Hook, J.B., Helmrath, T.A. et al., Effects of asphyxia on cardiac output and organ blood flow in the newborn piglet. Pediatric Research, 1978. 12(8): p. 824-827. 
129. Alward, C.T., Hook, J.B., Helmbrath, T.A. et al., Effects of asphyxia on renal function in the newborn piglet. Pediatric Research, 1978. 12(3): p. 225-228.

130. van Dijk, A.J., van Loon, J.P., Taverne, M.A. et al., Umbilical cord clamping in term piglets: a useful model to study perinatal asphyxia? Theriogenology, 2008. 70(4): p. 662-674.

131. Armstead, W.M., Mirro, R., Busija, D.W. et al., Postischemic generation of superoxide anion by newborn pig brain. American Journal of Physiology, 1988. 255(2 Pt 2): p. H401-403.

132. Laptook, A.R., Corbett, R.J., Nguyen, H.T. et al., Alterations in cerebral blood flow and phosphorylated metabolites in piglets during and after partial ischemia. Pediatric Research, 1988. 23(2): p. 206-211.

133. Adam, G., Joo, F., Temesvari, P. et al., Effects of acute hypoxia on the adenylate cyclase and evans blue transport of brain microvessels. Neurochemistry International, 1987. 10(4): p. 529-532.

134. Gillilan, L.A., Blood supply to brains of ungulates with and without a rete mirabile caroticum. Journal of Comparative Neurology, 1974. 1(153): p. 275-290.

135. Massoud, T.F., Vinters, H.V., Chao, K.H. et al., Histopathologic characteristics of a chronic arteriovenous malformation in a swine model: Preliminary study. American Journal of Neuroradiology, 2000. 21(7): p. 1268-1276.

136. Gillilan, L.A., Blood supply to brains of Ungulates with and without a rete mirabile Caroticum. J. Comp. Neur., 1974. 153(3): p. 275-290.

137. Lylyk, P., Vinuela, F., Vinters, H.V. et al., Use of a new mixture for embolization of intracranial vascular malformations. Preliminary experimental experience. Neuroradiology, 1990. 32(4): p. 304-310.

138. Arakawa, H., Murayama, Y., Davis, C.R. et al., Endovascular embolization of the swine rete mirabile with Eudragit-E 100 polymer. AJNR American Journal of Neuroradiology, 2007. 28(6): p. 1191-1196.

139. Haaland, K., Orderud, W.J. and Thoresen, M., The piglet as a model for cerebral circulation: an angiographic study. Biology of the Neonate, 1995. 68(1): p. 75-80.

140. Burbridge, B., Matte, G. and Remedios, A., Complex intracranial arterial anatomy in swine is unsuitable for cerebral infarction projects. Canadian Association of Radiologists JournalJournal De L Association Canadienne Des Radiologistes, 2004. 55(5): p. 326-329.

141. Helmy, M.M., Tolner, E.A., Vanhatalo, S. et al., Brain alkalosis causes birth asphyxia seizures, suggesting therapeutic strategy. Annals of Neurology, 2011. 69(3): p. 493-500.

142. Monod, N., Pajot, N. and Guidasci, S., The neonatal EEG: statistical studies and prognostic value in full-term and pre-term babies. Electroencephalography and Clinical Neurophysiology, 1972. 32(5): p. 529-544.

143. Pezzani, C., Radvanyi-Bouvet, M.F., Relier, J.P. et al., Neonatal electroencephalography during the first twenty-four hours of life in full-term newborn infants. Neuropediatrics, 1986. 17(1): p. 11-18.

144. Wertheim, D., Mercuri, E., Faundez, J.C. et al., Prognostic value of continuous electroencephalographic recording in full term infants with hypoxic ischaemic encephalopathy. Archives of Disease in Childhood, 1994. 71(2): p. F97-102.

145. Pressler, R.M., Boylan, G.B., Morton, M. et al., Early serial EEG in hypoxic ischaemic encephalopathy. Clinical Neurophysiology, 2001. 112(1): p. 31-37.

146. Del Río, R., Ochoa, C., Alarcon, A. et al., Amplitude integrated electroencephalogram as a prognostic tool in neonates with hypoxic-ischemic encephalopathy: A systematic review. PLoS One, 2016. 11.

147. Fellman, V. and Raivio, K.O., Reperfusion injury as the mechanism of brain damage after perinatal asphyxia. Pediatric Research, 1997. 41(5): p. 599-606.

148. Domoki, F., Zimmermann, A., Cserni, G. et al., Reventilation with room air or $100 \%$ oxygen after asphyxia differentially affects cerebral neuropathology in newborn pigs. Acta Paediatr, 2006. 95(9): p. 1109-1115. 
149. Faa, G., Fanos, V., Fanni, D. et al., Reoxygenation of asphyxiated newborn piglets: administration of $100 \%$ oxygen causes significantly higher apoptosis in cortical neurons, as compared to 21\%. BioMed Research International, 2014. 476349.

150. Munkeby, B.H., Borke, W.B., Bjornland, K. et al., Resuscitation with $100 \%$ O2 increases cerebral injury in hypoxemic piglets. Pediatr Res, 2004. 56(5): p. 783-790.

151. Wyllie, J., Bruinenberg, J., Roehr, C.C. et al., European Resuscitation Council Guidelines for Resuscitation 2015 Section 7. Resuscitation and support of transition of babies at birth. Resuscitation, 2015. 95: p. 249-263.

152. Gunn, A.J., Gunn, T.R., deHaan, H.H. et al., Dramatic neuronal rescue with prolonged selective heed cooling after ischemia in fetal lambs. Journal of Clinical Investigation, 1997. 99(2): p. 248-256.

153. Gunn, A.J., Bennet, L., Gunning, M.I. et al., Cerebral hypothermia is not neuroprotective when started after postischemic seizures in fetal sheep. Pediatric Research, 1999. 46(3): p. 274-280.

154. Shankaran, S., Laptook, A., Wright, L.L. et al., Whole-body hypothermia for neonatal encephalopathy: Animal observations as a basis for a randomized, controlled pilot study in term infants. Pediatrics, 2002. 110(2): p. 377-385.

155. Azzopardi, D., Brocklehurst, P., Edwards, D. et al., The TOBY Study. Whole body hypothermia for the treatment of perinatal asphyxial encephalopathy: a randomised controlled trial. Bmc Pediatrics, 2008. 8(17): p. 1-12.

156. Gluckman, P.D., Wyatt, J.S., Azzopardi, D. et al., Selective head cooling with mild systemic hypothermia after neonatal encephalopathy: multicentre randomised trial. Lancet, 2005. 365(9460): p. 663-670.

157. Shankaran, S., Laptook, A.R., Ehrenkranz, R.A. et al., Whole-body hypothermia for neonates with hypoxic-ischemic encephalopathy. New England Journal of Medicine, 2005. 353(15): p. 1574-1584.

158. Perlman, J.M., Wyllie, J., Kattwinkel, J. et al., Part 11: Neonatal resuscitation: 2010 International Consensus on Cardiopulmonary Resuscitation and Emergency Cardiovascular Care Science With Treatment Recommendations. Circulation, 2010. 122(16 Suppl 2): p. S516538.

159. Thoresen, M., Penrice, J., Lorek, A. et al., Mild hypothermia after severe transient hypoxiaischemia ameliorates delayed cerebral energy failure in the newborn piglet. Pediatric Research, 1995. 37(5): p. 667-670.

160. Thoresen, M., Simmonds, M., Satas, S. et al., Effective selective head cooling during posthypoxic hypothermia in newborn piglets. Pediatric Research, 2001. 49(4): p. 594-599.

161. Thoresen, M., Who should we cool after perinatal asphyxia? Seminars in Fetal and Neonatal Medicine, 2015. 20(2): p. 66-71.

162. Thoresen, M., Cooling after perinatal asphyxia. Seminars in Fetal and Neonatal Medicine, 2015. 20(2): p. 65.

163. Zhuang, L., Yang, T., Zhao, H. et al., The protective profile of argon, helium, and xenon in a model of neonatal asphyxia in rats. Critical Care Medicine, 2012. 40(6): p. 1724-1730.

164. Alderliesten, T., Favie, L.M.A., Neijzen, R.W. et al., Neuroprotection by Argon Ventilation after Perinatal Asphyxia: A Safety Study in Newborn Piglets (vol 9, e113575, 2014). PLoS One, 2015. 10(7).

165. Alderliesten, T., Favie, L.M.A., Neijzen, R.W. et al., Neuroprotection by argon ventilation after perinatal asphyxia: a safety study in newborn piglets. PLoS One, 2014. 9(12).

166. Dingley, J., Tooley, J., Liu, X. et al., Xenon ventilation during therapeutic hypothermia in neonatal encephalopathy: a feasibility study. Pediatrics, 2014. 133(5): p. 809-818.

167. Azzopardi, D., Robertson, N.J., Bainbridge, A. et al., Moderate hypothermia within $6 \mathrm{~h}$ of birth plus inhaled xenon versus moderate hypothermia alone after birth asphyxia (TOBY-Xe): a proof-of-concept, open-label, randomised controlled trial. Lancet Neurology, 2016. 15(2): p. 145-153. 
168. Cui, J., Chen, X., Zhai, X. et al., Inhalation of water electrolysis-derived hydrogen ameliorates cerebral ischemia-reperfusion injury in rats - a possible new hydrogen resource for clinical use. Neuroscience, 2016. 335: p. 232-241.

169. Christl, S.U., Murgatroyd, P.R., Gibson, G.R. et al., Production, metabolism, and excretion of hydrogen in the large-intestine. Gastroenterology, 1992. 102(4): p. 1269-1277.

170. Nakao, A., Zhan, J.H., Wang, Y.N. et al., Oral administration of hydrogen water prevents chronic allograft nephropathy. American Journal of Transplantation, 2009. 9: p. 660-661.

171. Zhai, X., Chen, X., Shi, J. et al., Lactulose ameliorates cerebral ischemia-reperfusion injury in rats by inducing hydrogen by activating Nrf2 expression. Free Radical Biology \& Medicine, 2013. 65: p. 731-741.

172. Nagatani, K., Nawashiro, H., Takeuchi, S. et al., Safety of intravenous administration of hydrogen-enriched fluid in patients with acute cerebral ischemia: initial clinical studies. Medical Gas Research, 2013. 3(13).

173. Ono, H., Nishijima, Y., Adachi, N. et al., A basic study on molecular hydrogen (H2) inhalation in acute cerebral ischemia patients for safety check with physiological parameters and measurement of blood H2 level. Medical Gas Research, 2012. 2(21).

174. Hayashida, K., Sano, M., Ohsawa, I. et al., Inhalation of hydrogen gas reduces infarct size in the rat model of myocardial ischemia-reperfusion injury. Biochemical and Biophysical Research Communications, 2008. 373(1): p. 30-35.

175. Ichihara, M., Sobue, S., Ito, M. et al., Beneficial biological effects and the underlying mechanisms of molecular hydrogen - comprehensive review of 321 original articles. Medical Gas Research, 2015. 5(12).

176. Liu, C.L., Zhang, K. and Chen, G., Hydrogen therapy: from mechanism to cerebral diseases. Medical Gas Research, 2016. 6(1): p. 48-54.

177. Ohno, K., Ito, M., Ichihara, M. et al., Molecular hydrogen as an emerging therapeutic medical gas for neurodegenerative and other diseases. Oxidative Medicine and Cellular Longevity, 2012.

178. Wang, F., Yu, G.A., Liu, S.Y. et al., Hydrogen-rich saline protects against renal ischemia/reperfusion injury in rats. Journal of Surgical Research, 2011. 167(2): p. E339-E344.

179. Wang, R.B., Wu, J.C., Chen, Z.L. et al., Postconditioning with inhaled hydrogen promotes survival of retinal ganglion cells in a rat model of retinal ischemia/reperfusion injury. Brain Research, 2016. 1632: p. 82-90.

180. Han, L., Tian, R.F., Yan, H.H. et al., Hydrogen-rich water protects against ischemic brain injury in rats by regulating calcium buffering proteins. Brain Research, 2015. 1615: p. 129-138.

181. Wang, X.F., Zhang, L.S., Zhao, W. et al., The protective effects of hydrogen on HO-1 expression in the brain after focal cerebral ischemia reperfusion in rats. Turkish Journal of Medical Sciences, 2016. 46(5): p. 1534-1539.

182. Liu, Y., Liu, W., Sun, X. et al., Hydrogen saline offers neuroprotection by reducing oxidative stress in a focal cerebral ischemia-reperfusion rat model. Medical Gas Research, 2011. 1(15).

183. Zhao, L., Chen, X., Dai, Q. et al., Role of FOXO3a in process of hydrogen-rich saline attenuating global cerebral ischemia-reperfusion injury in rats. Zhonghua Yi Xue Za Zhi., 2015. 95(6): p. 457-461.

184. Ge, P., Zhao, J., Li, S. et al., Inhalation of hydrogen gas attenuates cognitive impairment in transient cerebral ischemia via inhibition of oxidative stress. Neurological Research, 2012. 34(2): p. 187-194.

185. Hugyecz, M., Mracsko, E., Hertelendy, P. et al., Hydrogen supplemented air inhalation reduces changes of prooxidant enzyme and gap junction protein levels after transient global cerebral ischemia in the rat hippocampus. Brain Research, 2011. 1404: p. 31-38.

186. Domoki, F., Olah, O., Zimmermann, A. et al., Hydrogen is neuroprotective and preserves cerebrovascular reactivity in asphyxiated newborn pigs. Pediatric Research, 2010. 68(5): p. 387-392. 
187. Cai, J., Kang, Z., Liu, K. et al., Neuroprotective effects of hydrogen saline in neonatal hypoxiaischemia rat model. Brain Research, 2009. 1256: p. 129-137.

188. Matchett, G.A., Fathali, N., Hasegawa, Y. et al., Hydrogen gas is ineffective in moderate and severe neonatal hypoxia-ischemia rat models. Brain Research, 2009. 1259: p. 90-97.

189. Nakayama, M., Nakano, H., Hamada, H. et al., A novel bioactive haemodialysis system using dissolved dihydrogen $(\mathrm{H}-2)$ produced by water electrolysis: a clinical trial. Nephrology Dialysis Transplantation, 2010. 25(9): p. 3026-3033.

190. Kajiyama, S., Hasegawa, G., Asano, M. et al., Supplementation of hydrogen-rich water improves lipid and glucose metabolism in patients with type 2 diabetes or impaired glucose tolerance. Nutrition Research, 2008. 28(3): p. 137-143.

191. Ono, H., Nishijima, Y., Adachi, N. et al., Improved brain MRI indices in the acute brain stem infarct sites treated with hydroxyl radical scavengers, Edaravone and hydrogen, as compared to Edaravone alone. A non-controlled study. Medical Gas Research, 2011. 1(12).

192. Yoritaka, A., Abe, T., Ohtsuka, C. et al., A randomized double-blind multi-center trial of hydrogen water for Parkinson's disease: protocol and baseline characteristics. Bmc Neurology, 2016. 17(35).

193. Xia, C.X., Liu, W.W., Zeng, D.X. et al., Effect of hydrogen-rich water on oxidative stress, liver function, and viral load in patients with chronic hepatitis B. Cts-Clinical and Translational Science, 2013. 6(5): p. 372-375.

194. Ishibashi, T., Sato, B., Shibata, S. et al., Therapeutic efficacy of infused molecular hydrogen in saline on rheumatoid arthritis: A randomized, double-blind, placebo-controlled pilot study. International Immunopharmacology, 2014. 21(2): p. 468-473.

195. Ito, M., Ibi, T., Sahashi, K. et al., Open-label trial and randomized, double-blind, placebocontrolled, crossover trial of hydrogen-enriched water for mitochondrial and inflammatory myopathies. Medical Gas Research, 2011. 1(24).

196. Casey, J.R., Grinstein, S. and Orlowski, J., Sensors and regulators of intracellular $\mathrm{pH}$. Nature Reviews Molecular Cell Biology, 2010. 11(1): p. 50-61.

197. Tang, C.M., Dichter, M. and M., M., Modulation of the N-methyl-D-aspartate channel by extracellular $\mathrm{H}+$. Proceedings of the National Academy of Sciences of the USA., 1990. 87(16): p. 6445-6449.

198. Tombaugh, G.C. and Somjen, G.G., Effects of extracellular $\mathrm{pH}$ on voltage-gated $\mathrm{Na}+\mathrm{K}+$ and Ca2+ currents in isolated rat CA1 neurons. The Journal of Physiology, 1996. 493 (3): p. 719732.

199. Bender, T.M., Johnston, J.A., Manepalli, A.N. et al., Association between brain tissue $\mathrm{pH}$ and brain injury during asphyxia in piglets. Resuscitation, 2003. 59(2): p. 243-254.

200. Moon, R.B. and Richards, J.H., Determination of intracellular $p H$ by 31P MR. The Journal of Biological Chemistry, 1973. 248(20): p. $7276-7278$.

201. Hope, P.L., Costello, A.M., Cady, E.B. et al., Cerebral energy metabolism studied with phosphorus NMR spectroscopy in normal and birth-asphyxiated infants. Lancet, 1984. 2(8399): p. 366-370.

202. Robertson, N.J., Cowan, F.M., Cox, I.J. et al., Brain alkaline intracellular $p H$ after neonatal encephalopathy. Annals of Neurology, 2002. 52(6): p. 732-742.

203. Chopp, M., Chen, H., Vande Linde, A.M. et al., Time course of postischemic intracellular alkalosis reflects the duration of ischemia. Journal of Cerebral Blood Flow and Metabolism, 1990. 10(6): p. 860-865.

204. Thoresen, M., Satas, S., Loberg, E.M. et al., Twenty-four hours of mild hypothermia in unsedated newborn pigs starting after a severe global hypoxic-ischemic insult is not neuroprotective. Pediatric Research, 2001. 50(3): p. 405-411.

205. Bona, E., Hagberg, H., Loberg, E.M. et al., Protective effects of moderate hypothermia after neonatal hypoxia-ischemia: Short- and long-term outcome. Pediatric Research, 1998. 43(6): p. 738-745. 
206. Hall, R.W. and Shbarou, R.M., Drugs of choice for sedation and analgesia in the neonatal ICU. Clinics in Perinatology, 2009. 36(1): p. 15-26.

207. Aranda, J.V., Carlo, W., Hummel, P. et al., Analgesia and sedation during mechanical ventilation in neonates. Clinical Therapeutics, 2005. 27(6): p. 877-899.

208. Olischar, M., Davidson, A.J., Lee, K.J. et al., Effects of morphine and midazolam on sleep-wake cycling in amplitude-integrated electroencephalography in post-surgical neonates $>/=32$ weeks of gestational age. Neonatology, 2012. 101(4): p. 293-300. 
I 


\title{
COMPARISON OF CEREBROCORTICAL MICROVASCULAR EFFECTS OF DIFFERENT HYPOXIC-ISCHEMIC INSULTS IN PIGLETS: A LASER-SPECKLE IMAGING STUDY
}

\author{
${ }^{1}$ Department of Physiology, Faculty of Medicine, University of Szeged, Szeged, Hungary; \\ ${ }^{2}$ Department of Optics and Quantum Electronics, University of Szeged, Szeged, Hungary; \\ ${ }^{3}$ Department of Medical Physics and Informatics, Faculty of Medicine, University of Szeged, Szeged, Hungary; \\ ${ }^{4}$ MTA-SZTE Research Group on Photoacoustic Spectroscopy, University of Szeged, Szeged, Hungary
}

\begin{abstract}
The newborn pig is a widely accepted large animal model of hypoxic/ischemic (H/I) encephalopathy (HIE) of the term neonate appropriate for translational research. The methodology of the induction of H/I stress shows extensive variability of the literature, and little is known how these affect study outcome. The purpose of the present study was to determine the cerebrocortical microvascular effects of different H/I insults used in current HIE piglet models. For the semiquantitative study of cerebrocortical blood flow, we developed a methodological innovation: an operating microscope was converted into a custom-designed laser-speckle imager. Anesthetized, air-ventilated newborn pigs $(n=7)$ were fitted with a closed cranial window. Speckle image series $(2 \mathrm{~ms}, 1 \mathrm{~Hz})$ were collected during baseline conditions, during transient bilateral carotid artery occlusion $(\mathrm{BCAO})$, hypoxic $\left(\mathrm{FiO}_{2}=0.1\right)$ hypoxia, hypoxia $+\mathrm{BCAO}$, and asphyxia induced by suspending ventilation. Laser-speckle contrast analysis was performed off-line over parenchymal and arteriolar regions of interests, and pial arteriolar diameters were also determined for detailed analysis of cortical perfusion changes. Under normoxic conditions, transient BCAO did not affect parenchymal perfusion or pial arteriolar diameters. Hypoxia induced marked cortical hyperemia in 5 out of 7 piglets, with simultaneous increases in pial arteriolar diameters and arteriolar flow velocity, however, BCAO could not even affect these hypoxia-induced perfusion changes. In contrast to hypoxia or hypoxia $+\mathrm{BCAO}$, asphyxia inevitably led also to severe cerebrocortical ischemia. In summary, acute reversible BCAO does not reduce cerebrocortical blood flow in the piglet, and thus it likely does not exacerbate the effect of hypoxic ventilation. Asphyxia elicits not only severe hypoxia, but also severe brain ischemia. These microcirculatory effects must be taken into consideration when assessing results obtained in the various HIE piglet models.
\end{abstract}

Key words: carotid artery, cranial window, cerebrovascular reactivity, autoregulation, pial arterioles, brain ischemia

\section{INTRODUCTION}

The newborn piglet is an accepted large animal model to study the pathophysiology of hypoxic/ischemic (H/I) encephalopathy (HIE) of term infants, and to test putative neuroprotective strategies. HIE has multiple aetiology, and similar to this clinical heterogeneity, there have been numerous experimental methods in use to induce $\mathrm{H} / \mathrm{I}$ stress in the newborn piglet. Although in the last three decades many experimental approaches have been tested ranging from acute total global cerebral ischemia induced by elevation of intracranial pressure above arterial pressure (1) to asphyxia induced by bilateral pneumothorax (2), however, more recently $\mathrm{H} / \mathrm{I}$ stress is elicited by ventilating the piglets with hypoxic gas mixtures with (3-5) or without (6-8) bilateral occlusion of the common carotid arteries, suspending ventilation by occluding the endotracheal tube to elicit asphyxia (9), or sequential combination of the two methods (hypoxia/reoxygenation followed by asphyxia/reventilation) $(10,11)$. Understandably, all research groups adhere to their preferred $\mathrm{H} / \mathrm{I}$ induction method in order to inflict consistent neurological damage and to be able to compare their results with the outcome of previous experiments. Therefore, direct comparisons between these H/I models are very scarce in the literature, and there is virtually no information to which extent these models actually elicit blood flow decreases in the brain, although the degree of ischemia is indeed a key determinant of neuronal damage.

In the present study, we set out to investigate the cerebrocortical microcirculatory effects of $\mathrm{H} / \mathrm{I}$ insults that are often used in piglet models of HIE. More specifically, we were interested (I) how bilateral carotid artery occlusion (BCAO) affected the cortical perfusion in normoxic and in hypoxic conditions, and (II) how cerebrocortical perfusion altered during asphyxia/reventilation. We used the closed cranial window/laserspeckle imaging (LSI) technique that produces vivid twodimensional perfusion maps, and these images were then evaluated with laser speckle contrast analysis (LASCA) allowing simultaneous assessment of parenchymal perfusion, pial arteriolar diameter and arteriolar flow velocity changes as shown previously (12). 


\section{MATERIALS AND METHODS}

Animals

Newborn (1-2 days old, body weight: 1.5-2.5 kg) male Large-White piglets $(n=7)$ were obtained on the day of experimentation from a local company (Pigmark Ltd. Co., Szeged, Hungary). All procedures were approved by the Animal Care and Use Committee of the University of Szeged.

Anesthesia was induced with intraperitoneal injection of sodium thiopental ( $45 \mathrm{mg} / \mathrm{kg}$; Sandoz, Kundl, Austria) followed by intubation through tracheotomy and artificial ventilation with warmed and humidified medical air $\left(21 \% \mathrm{O}_{2}\right.$, balance $\left.\mathrm{N}_{2}\right)$, using a pressure-controlled ventilator. The ventilation rate (25-35/min) and the peak inspiratory pressure (100-125 $\mathrm{mmH}_{2} \mathrm{O}$ ) were set to keep blood gases and oxygen saturation in the physiological range. To elicit transient $\mathrm{BCAO}$, remotely controlled vascular occlusion cuffs (OC 2A, In Vivo Metric, Healdsburg, CA, USA) were secured around both exposed common carotid arteries. The right femoral artery and vein were catheterized to monitor arterial blood pressure, $\mathrm{pH}, \mathrm{pCO}_{2}, \mathrm{pO}_{2}$, glucose, and to inject drugs and fluids, respectively. Anesthesia/analgesia was continued with intravenous bolus injection of morphine $(100 \mu \mathrm{g} / \mathrm{kg}$; Teva, Petah Tikva, Israel), and midazolam $(250 \mu \mathrm{g} / \mathrm{kg}$; Torrex Pharma, Vienna, Austria) then maintained with intravenous infusion of morphine $(10 \mu \mathrm{g} / \mathrm{kg} / \mathrm{h})$, and midazolam $(250 \mu \mathrm{g} / \mathrm{kg} / \mathrm{h})$ along with fluids ( $5 \%$ glucose, $0.45 \% \mathrm{NaCl}, 2-5 \mathrm{ml} / \mathrm{kg} / \mathrm{h}$ ), with additional boluses if necessary. Body temperature was kept in the physiological range $\left(38.5 \pm 0.5^{\circ} \mathrm{C}\right)$ using an electric heating pad.

The animals were then placed in a prone position with the head fixed in a stereotactic frame. Oxygen saturation, heart rate, arterial blood pressure, and body temperature were monitored with a Hewlett-Packard M1094 monitor (Palo Alto, California, USA); the data were on-line recorded using a PC (MecifView, Arlington, MA, USA). After retraction of the scalp, a $\sim 20 \mathrm{~mm}$ diameter circular craniotomy was made in the left parietal bone, where a stainless steel closed cranial window with three needle ports was inserted after careful removal of the dura mater. The cranial window was sealed with bone wax, cemented in place with dental acrylic, and was filled with artificial cerebrospinal fluid (aCSF; containing $\mathrm{KCl} 220, \mathrm{MgCl}_{2} 132, \mathrm{CaCl}_{2} 221, \mathrm{NaCl}$ 7710, urea 402, dextrose 665, and $\mathrm{NaHCO}_{3} 2066 \mathrm{mg} / 1$, warmed to $37^{\circ} \mathrm{C}$ and equilibrated with a gas mixture containing $6 \% \mathrm{O}_{2}$, $6.5 \% \mathrm{CO}_{2}$ and $87.5 \% \mathrm{~N}_{2}$ ). There was a 60 min stabilization period allowed after the implantation of the cranial window before commencing the experiments.

The experimental protocol was the following: after obtaining 2 min baseline, transient BCAO was elicited by inflating the occluders with air to $280 \mathrm{mmHg}$ for $2 \mathrm{~min}$. $5 \mathrm{~min}$ after the termination of the first $\mathrm{BCAO}, \mathrm{FiO}_{2}$ was reduced to 0.1. Two more BCAOs (2-2 min) were elicited at the $5^{\text {th }}$ and $30^{\text {th }}$ minutes of hypoxic period. Five min after the last occlusion period, the animals were reoxygenated by ventilating them again with air for $5 \mathrm{~min}$, followed by $7 \mathrm{~min}$ asphyxia induced by halting artificial ventilation and blocking the endotracheal tube. After asphyxia, the animals were reventilated with air for 5 min then the anesthetized animals were euthanized to obtain biological zero measurements. During these interventions LSI was performed, and the obtained images were analyzed by LASCA as described in the following section.

\section{Laser-speckle imaging and contrast analysis}

The cranial window was illuminated by the polarized light of a near infrared diode laser $(\lambda=808 \mathrm{~nm}, 200 \mathrm{~mW}$; DL-8141-002 SANYO Electric Co., Japan) through an optical polarizing cube beamsplitter ( $850 \mathrm{~nm}, 35 \mathrm{~mm}$; Edmund Optics Ltd, York, UK) approximately perpendicular to its surface (Fig. 1). The speckle images were recorded through the same polarizing cube that was attached through a custom-made bayonet adaptor to the objective of an operating microscope (Wild, Heerburg, Switzerland), coupled to a $1280 \times 1024$ pixel monochrome camera (PL-B741F; PixeLINK® Ottawa, Canada). Speckle images were recorded with $2 \mathrm{~ms}$ exposure time with 1 frame/s rate, and stored on a personal computer. Speckle contrast analysis was performed off line using custom-designed software written in LabVIEW (National Instruments Co., Austin, TX, USA). The local contrast maps were calculated from the recorded speckle images using rolling windows of $5 \times 5$ pixel areas. For each measurement in each animal, 4-4 regions of interests (ROIs) having an area of $5 \times 5$ pixels $\left(\sim 100 \mu \mathrm{m}^{2}\right)$ on the raw speckle images were identified over the cortical parenchyma and over pial arterioles. The $\tau$ correlation times were calculated using eq. 1 , where $\mathrm{K}$ is the average speckle contrast of the corresponding ROI, $\mathrm{T}$ is the exposure time and $\beta$ is the coherence factor:

$$
K(T)=\sqrt{\beta}\left\{\frac{\tau^{2}}{2 T^{2}}\left[\exp \left(\frac{-2 T}{\tau}\right)-1+\frac{2 T}{\tau}\right]\right\}^{1 / 2} \quad 1 .
$$

The $\beta$ coherence factor was previously determined from the speckle contrast of images recorded from a white Teflon ${ }^{\circledR}$ sheet as proposed in the literature (13). The $1 / \tau$ values were normalized to the respective baseline values as presented in the Results. $1 / \tau$ over parenchymal ROIs were determined in every image, however, in order to obtain better spatial resolution of arterioles, $1 / \tau$ values of ROIs over arterioles were determined at selected timepoints representing steady states of $30 \mathrm{~s}$ periods after averaging the contrast map of 31 consecutive images in order to decrease the native statistical noise of the images. On these images the internal diameter of the pial arterioles was also determined.

\section{Statistical analysis}

Parametric data are expressed as mean \pm S.E.M. Data were analyzed with statistical software (SigmaPlot 11, Systat Software Inc., San Jose, CA, USA). Data were analyzed with one-way repeated measures ANOVA, followed by the StudentNewman-Keuls post hoc test where appropriate. P values $<0.05$ were considered statistically significant.

\section{RESULTS}

The assessed physiological parameters were in the normal range in all animals before the onset of hypoxia, the values were: MABP: $79 \pm 3 \mathrm{mmHg}$; heart rate $163 \pm 111 / \mathrm{s}$; core body temperature $38.5 \pm 0.1^{\circ} \mathrm{C} ; \mathrm{pH}: 7.42 \pm 0.03 ; \mathrm{pCO}_{2}: 38 \pm 3 \mathrm{mmHg}$, $\mathrm{pO}_{2}: 79 \pm 8 \mathrm{mmHg}$, oxygen saturation: $94 \pm 1 \%$; glucose: $5.4 \pm 0.6$ $\mathrm{mmol} / \mathrm{L}$. Hypoxia decreased oxygen saturation to $37 \pm 4 \%$, and after 30 min blood gas values were: $\mathrm{pH}$ : $7.29 \pm 0.04 ; \mathrm{pCO}_{2}: 37 \pm 2$ $\mathrm{mmHg}, \mathrm{pO}_{2}: 22 \pm 2 \mathrm{mmHg}$. The systemic cardiovascular response to hypoxia was quite variable: MABP was maintained or even increased in 5 piglets (from $78 \pm 12$ to $85 \pm 19 \mathrm{mmHg}$ ), however, in two animals, MABP was severely depressed: from 84 to 51 and from 71 to $39 \mathrm{mmHg}$, respectively. Asphyxia resulted in severe acidosis, hypercapnia, hypoxia: the values were: $\mathrm{pH}: 6.73 \pm 0.02 *$; $\mathrm{pCO}_{2}: 87 \pm 6^{*} \mathrm{mmHg}, \mathrm{pO}_{2}: 14 \pm 3 * \mathrm{mmHg}$, respectively (* significantly different from baseline values).

BCAO did not affect cortical parenchymal perfusion in either normoxic conditions (Figs. $2 A, 3 A, 3 B$ ), or the early/late phase of hypoxic ventilation (Fig. $2 B, 2 C$ ). Hypoxia elicited in 


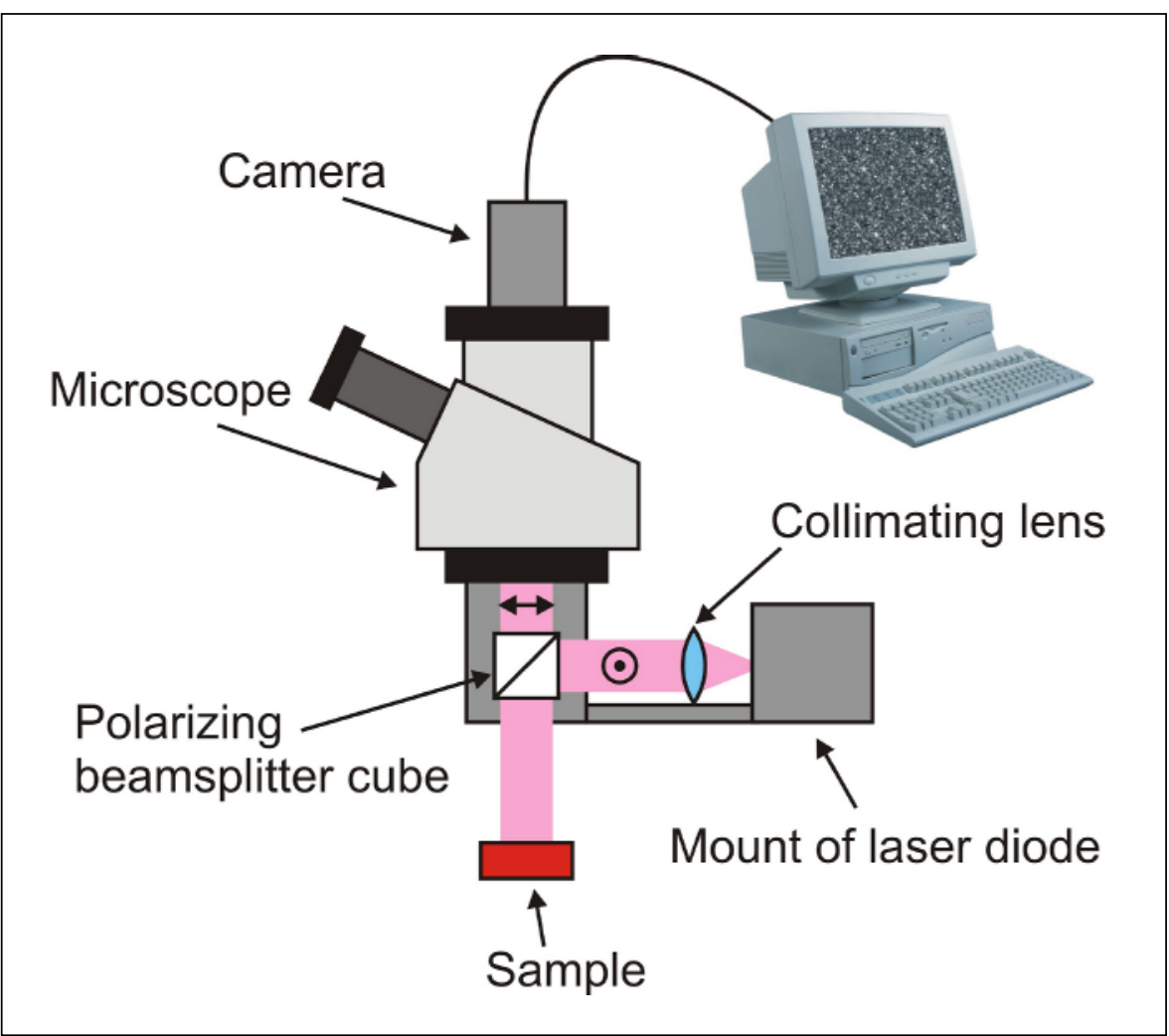

Fig. 1. The technical setup of the LSI device. The S-polarized light (direction of polarization is indicated by $\odot$ ) of the laser diode was collimated, and then reflected towards the sample by the polarizing beamsplitter cube. The polarizing beamsplitter was designed as to reflect one polarization direction (S) while to transmit the other one (P-polarized light indicated by $\leftrightarrow$ ). The light which was backscattered from the sample was depolarized (non-polarized) due to the multiple scattering, however, only its P-polarized components could reach the microscope and the camera through the cube. This enhanced the contrast of the speckle images by cutting off any laser photons suffering direct reflection from the optical components, while allowing only the light scattered from the monitored sample towards the microscope. marked increases in cortical perfusion in the animals that maintained or increased their MABP after the initiation of hypoxia (Figs. 2B, 3C, 3D). In these animals, the hyperaemic response to hypoxia reached a steady state approximately after $\sim 10 \mathrm{~min}$ and the dynamics of this blood flow increase appeared unaffected by the onset of the $2^{\text {nd }} \mathrm{BCAO}$ period elicited $5 \mathrm{~min}$ after the induction of hypoxia (Fig. 2B). In the two animals responding with hypotension, at the $10^{\text {th }}$ minute of hypoxia cortical perfusion was reduced to $63 \%$ and $62 \%$ of baseline values, respectively. After the restoration of normoxia, elevated cortical perfusion corresponding with reactive hyperemia was observed, however, asphyxia quickly resulted in severe, but upon reventilation reversible cortical ischemia (Figs. 2D, 3E).

BCAO did not significantly affect pial arteriolar diameters, but it elicited a small increase in the velocity of arteriolar flow (Fig. 4A, 4B). In contrast to the minor effect of BCAO on arterioles, in the animals that maintained/increased MABP to hypoxia, hypoxia elicited a robust sustained vasodilation and a parallel increase in arteriolar flow velocity (Fig. $4 A, 4 B$ ). In the two animals responding with hypotension, hypoxic vasodilation was less prominent, and arteriolar $1 / \tau$ values were also reduced to $56 \%$ and $57 \%$ of baseline values (at $10^{\text {th }} \mathrm{min}$ of hypoxia), respectively. Asphyxia elicited a $\sim 90 \%$ but upon ventilation reversible decrease in arteriolar flow velocities again corresponding well with the ischemia/reperfusion observed in the cortical parenchyma.

\section{DISCUSSION}

The major novel finding of the present study is that BCAO does not significantly reduce cerebrocortical perfusion, and it does not even reduce hypoxia-induced hyperemia in the cortical microcirculation. We also demonstrated that asphyxia indeed elicits severe cerebrocortical ischemia.
Choosing an appropriate animal model is of vital importance for successful translational research. In rodents, unilateral carotid artery occlusion combined with hypoxia produces robust, unilateral infarction that has been widely utilized (14-16). In contrast to the neonatal rodents with premature lyssencephalic brains, the newborn pig has long been recognized as one of the best relatively easily available large animal model of the term human neonate to study HIE (17). This statement, however, refers primarily to the piglet's brain, but not to its supplying arteries. In addition to differences in the anatomy of the origin of the common carotid arteries, there is a so-called rete mirabile at the base of the brain providing extensive anastomoses among all major extracranial arteries that serve the brain. The compensatory capacity of the rete mirabile in newborn pig HIE models remained undetermined, although in adult swine it has become an important neurosurgery model of arteriovenous malformations (18). Haaland et al. published a detailed angiographic study of the piglet extracerebral vasculature, in which they carefully proposed that ligation of any extracranial arteries likely would not induce severe focal cerebral ischemia (19). In agreement with this suggestion, unilateral carotid artery occlusion was indeed demonstrated not to affect cerebral perfusion (20), but the efficacy of BCAO to elicit cerebral ischemia augmenting the insult of simultaneous hypoxic ventilation was not questioned previously. Even very recently, a series of very important high-impact studies have been published using hypoxic ventilation with BCAO in the piglet (21-24), however, in these studies cerebrocortical blood flow was not assessed.

In our present paper, we utilized LSI/LASCA, a very sensitive novel method to detect cerebrocortical perfusion changes triggered by $\mathrm{BCAO}$, hypoxia, hypoxia $+\mathrm{BCAO}$, and asphyxia. We demonstrated previously that LSI/LASCA could record cerebrocortical parenchymal perfusion increases or decreases to a number of vasoactive stimuli that match blood flow changes 
measured by other methods (12). The present study yields novel insight on the unexpected inability of $\mathrm{BCAO}$ to reduce cerebrocortical perfusion, although it is well-known that in rats BCAO leads to chronic cerebral hypoperfusion $(25,26)$. In fact, BCAO did not even blunt the hyperemic response to hypoxia. In this study, we performed relatively short duration transient BCAOs, not as long ones used to induce HIE. However, this is clearly not a limitation, since our purpose was to test the microcirculatory effect of a sudden drop in perfusion pressure induced by BCAO. The ischemia induced by such vascular occlusion is expected to take place immediately and reach its maximum within a few seconds before the perfusion would start to recover due to the activation of autoregulation mechanisms (27). However, we saw no signs of this sudden decrease in any of our experiments. We repeated the BCAO challenge after $30 \mathrm{~min}$ of hypoxia, where conceivably due to the hypoxic vasodilation the compensatory capacity of the microcirculatory bed can be impaired to withstand a drop in perfusion pressure, however,

Protocol timeline with figure panels (A-D):

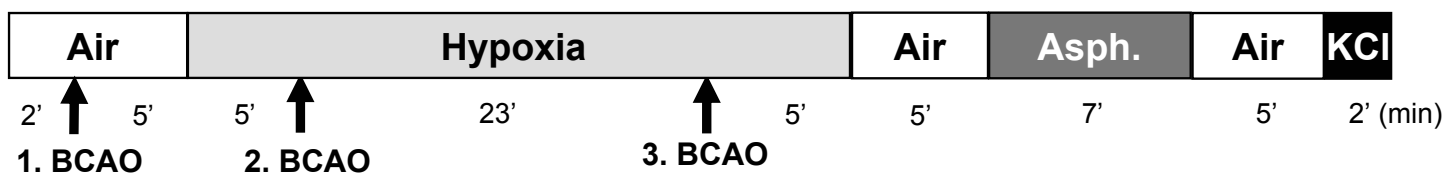

$A \quad B$

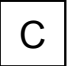

D
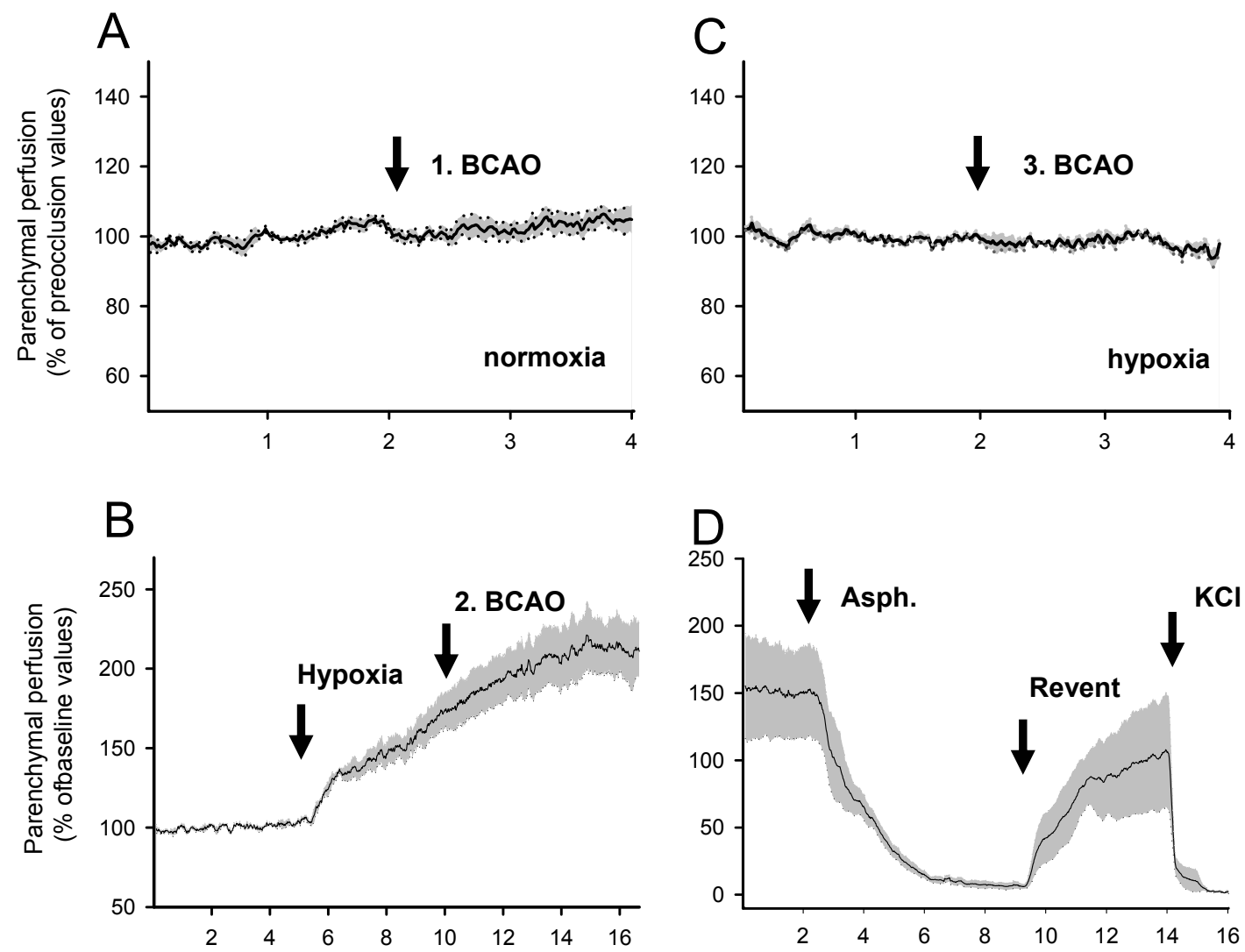

time ( $\min )$

Fig. 2. Cortical parenchymal perfusion assessed with LASCA. The timeline of the experimental protocol is shown together with the time periods plotted on Panels $A-D$. Transient BCAO was performed three times, once under mechanical ventilation with air (Air), and twice during ventilation with $10 \% \mathrm{O}_{2}$ (hypoxia). After 5 min reoxygenation mechanical ventilation was suspended to induce asphyxia (Asph.). After reventilation biological zero was obtained after iv $\mathrm{KCl}$ solution $(\mathrm{KCl})$. Cortical parenchymal perfusion is expressed as the \% change in normalized $1 / \tau$ values compared to the respective baseline (Panels $A, B, D$ ) or before BCAO (Panel $C$ ) measurements. The mean is plotted as the solid line, the S.E.M. as the grey shaded area. BCAO did not affect cortical perfusion during steady states either under normoxic (Panel $A$ ) or hypoxic (Panel $C$ ) conditions. Furthermore, BCAO did not modify the development of hyperemia after the induction of hypoxia in the 5 animals that could maintain their MABP during the hypoxic period (Panel $B$ ). Reoxygenation lead to variable $(\sim 50 \%)$ reactive hyperemia, then asphyxia resulted in severe but reversible ischemia that developed over the first 4-5 min of the period. Cardiac arrest resulting in the loss of perfusion pressure resulted in an instant complete ischemia (Panel $D)$. 
BCAO was also remarkably ineffective in this condition as well. This intriguing independence of the piglet cerebral circulation from the patency of the common carotid arteries likely stems principally from the rete mirabile anastomotic network, but also the extracranial branches of the common carotid/internal carotid arteries (more specifically the external carotid, occipital and condylic arteries) can retrogradely fill the internal carotid artery after BCAO that is typically performed at the level of the $4^{\text {th }}$ cervical vertebra (22) thus proximal from these branches (19). Although it might be possible to obtain a "modified" carotid artery by ligating all these branches (19), this would require quite extensive surgery and have not been utilized in any piglet HIE models yet. Our present findings in the piglet are consistent with those demonstrated in other ungulates, specifically ruminants: sheep and calves (28). These species also have a rete mirabile and an extensive extracranial (occipitovertebral) anastomotic network, and $\mathrm{BCAO}$ was shown to be compensated by flow increases in remaining patent arteries (28). Importantly, in fetal sheep HIE models, the ligation of occipitovertebral anastomoses is routinely used to enable BCAO to elicit brain ischemia (29).
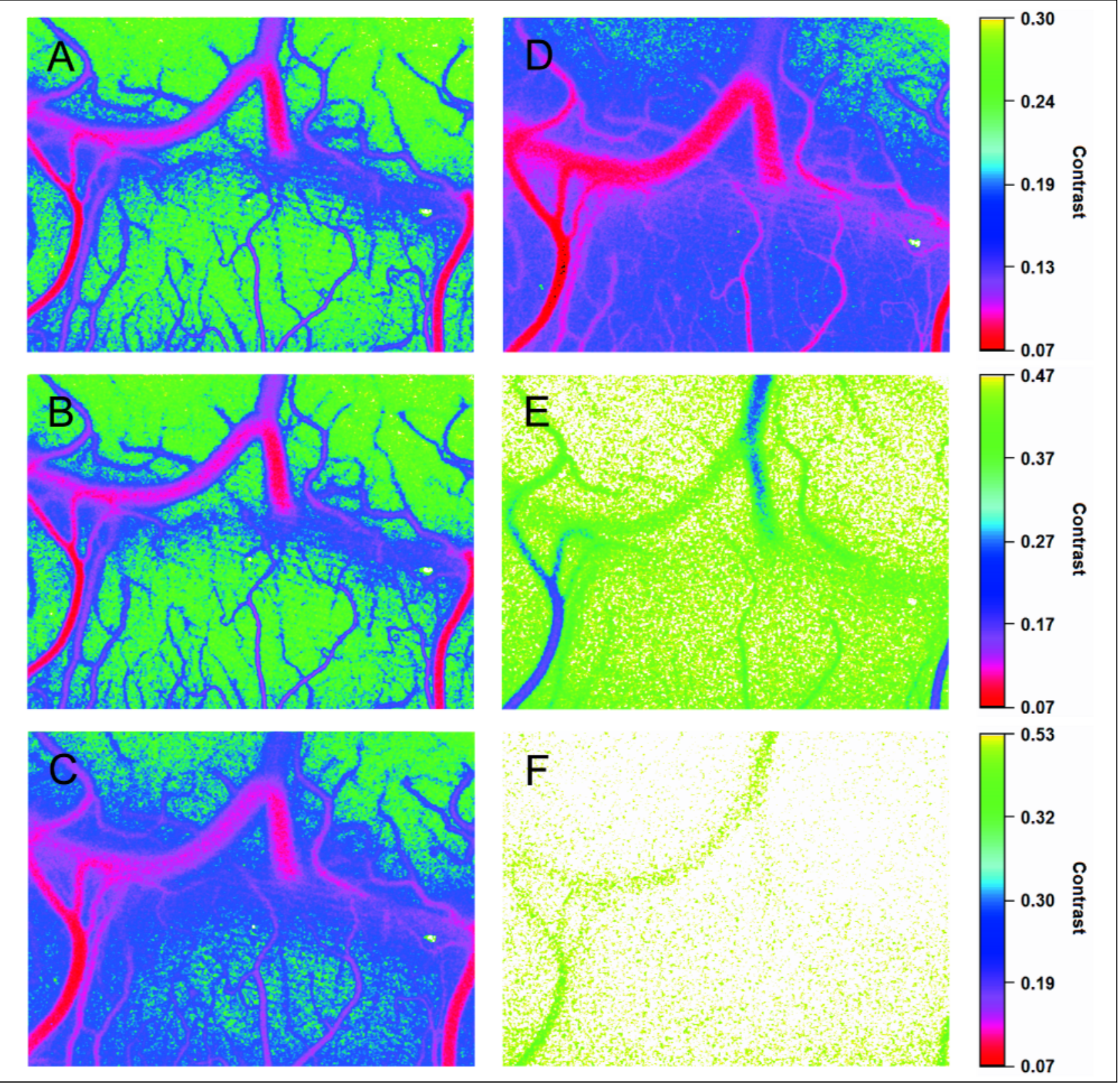

Fig. 3. Representative laser-speckle contrast images obtained through a closed cranial window from piglet experiment \#802 (A-F). Speckle contrast images provide vivid perfusion maps of the cerebrocortical surface, with lower contrast values in the high velocity pial vessels, and higher contrast values for the parenchyma representing slower velocity capillary flow. Corresponding contrast scales on the right: Panels $A-D$ : upper, Panel $E$ : middle, Panel $F$ : lower scale. Panel $A$ : baseline condition. Panel $B$ : BCAO does not substantially change the perfusion map. Panels $C-D: 10-30 \mathrm{~min}$ after the initiation of hypoxia, reduction in parenchymal speckle contrast, and arteriolar vasodilation can be easily observed representing maintained hypoxic hyperemia. Panel $E$ : asphyxia (7 min) will result in severe ischemia shown by the robust increase in speckle contrast. In order to visualize the remnant perfusion, the contrast scale had to be reset. Panel $F$ : after euthanasia, the speckle contrast further increases with the simultaneous disappearance of the perfusion map. 

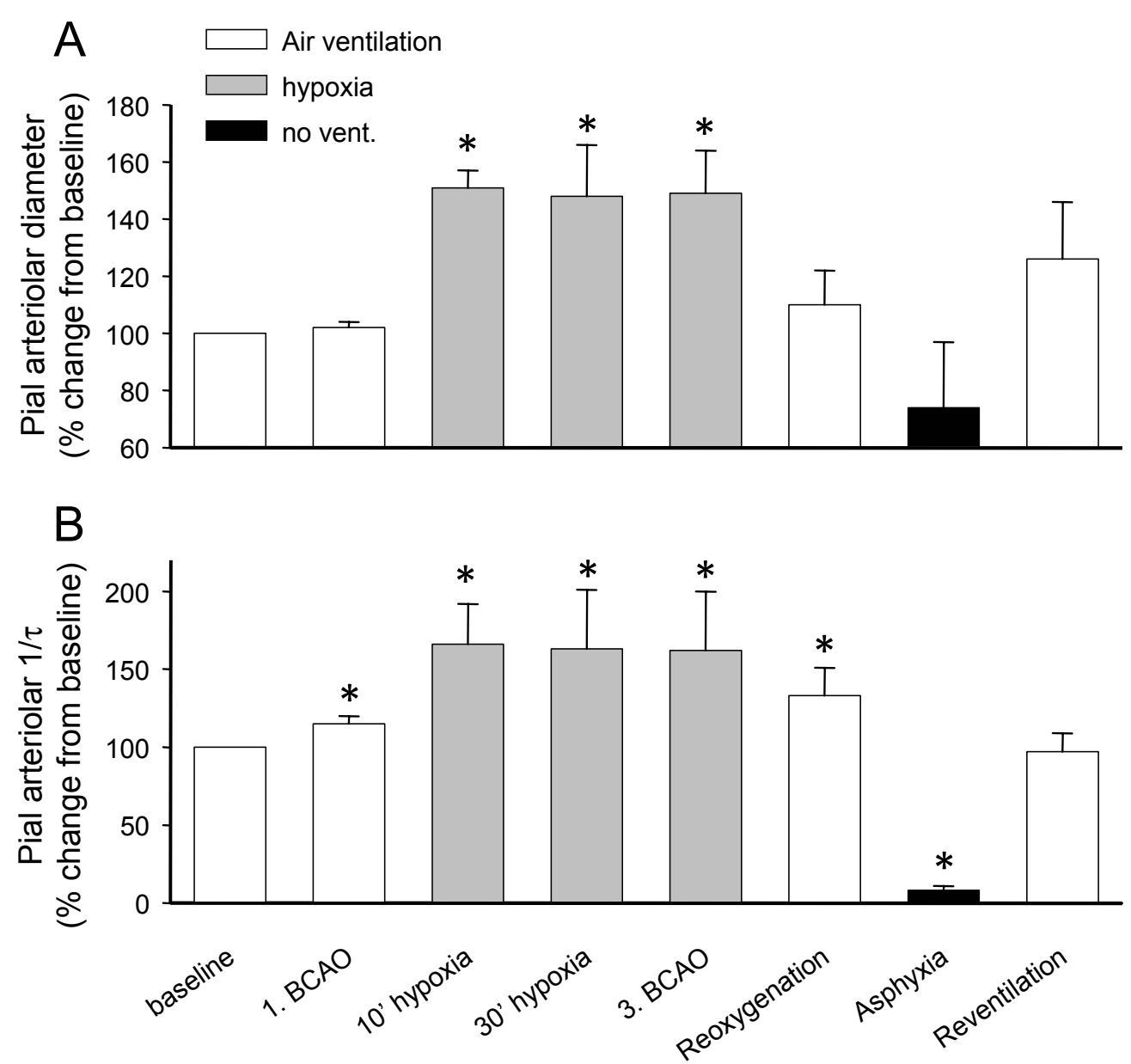

Fig. 4. Pial arteriolar diameters determined on laser-speckle contrast images (Panel $A$ ) and arteriolar flow velocities (Panel $B)$ assessed with LASCA at steady state time points. Data are expressed as the $\%$ change from baseline. Under normoxic conditions, BCAO did not affect pial arteriolar diameters but resulted in a small increase in arteriolar flow velocity. Hypoxia, however, elicited robust, maintained arteriolar vasodilation accompanied by increased arteriolar flow velocities that was not modified by BCAO. Asphyxia resulted in cortical ischemia, with barely detectible arteriolar flow. ${ }^{*} \mathrm{p}<0.05$, significantly different from baseline, $\mathrm{n}=5-7$.

A certain limitation of the present study is that perfusion of subcortical structures was not assessed, however, it is well known that subcortical forebrain and brainstem structures have even better autoregulation than the cortex (30). Since the cortical blood flow change was virtually zero after BCAO, we can safely expect the same for the other brain regions as well. This conclusion is also supported by a magnetic resonance imaging study, where reduction in regional cerebral blood flow in response to hypoxic ventilation with $\mathrm{BCAO}$ was found not to correlate with the success of BCAO (31). Accordingly, BCAO should not be expected to worsen the neurological damage induced by hypoxic ventilation alone, and although such comparative studies are very scarce in the literature, at least one previous study found no difference between these models concluding that BCAO was not necessary (32). Our present findings fully support this notion providing hemodynamic evidence on the lack of developing ischemia during hypoxic ventilation + BCAO resulting in a H/I stress of similar severity to hypoxic ventilation alone.

In contrast to hypoxia $+\mathrm{BCAO}$, asphyxia induced by suspended ventilation results in severe ischemia, perfusion decreases by $80-90 \%$ in accordance with our previous study with laser-Doppler flowmetry (LDF) (33). We would like to emphasize however, that LSI/LASCA compared to single-point LDF enabled us to select multiple and more precise parenchymal ROIs in each animals, thus resulting in less variable data while maintaining the excellent temporal resolution of the measurements. In addition LSI/LASCA also provides means to follow diameter changes of pial arterioles similar to intravital microscopy. Our present study showed that pial arteriolar diameter changes correspond well with parencymal perfusion alterations indicating an important role for this segment of resistance vessels in regulating cortical blood flow.

It is noteworthy that we created a novel LSI imager for the present study. Our relatively simple technical innovation can transform virtually any operating microscope equipped with a sufficient video camera into a LSI device. The advantages of this arrangement are twofold (in addition to the low cost): (I) the light source is stabilized with respect to the objective, so the same illumination of the cranial window is assured in every experiment; (II) the attached optical cube can be quickly mounted/dismounted thus the traditional use of the microscope remains possible.

In conclusion, BCAO does not elicit cortical ischemia making this intervention unnecessary to be included in the methodology of piglet HIE models. HIE models that combine 
hypoxic ventilation with systemic hypotension or induce asphyxia $(11,34)$, appear to better reproduce the ischemic aspect of HIE in this species.

FD and DZ-S contributed equally to this manuscript.

Acknowledgments: Financial support: National Scientific Research Fund of Hungary (OTKA, K81266, K100851). Orsolya Olah was supported through the TAMOP-4.2.2/B-10/12010-0012 grant. Ferenc Domoki was supported by the Janos Bolyai Research Scholarship of the Hungarian Academy of Sciences and through the TAMOP-4.2.2/A-11/1/KONV-20100052 grant.

Conflict of interest: None declared.

\section{REFERENCES}

1. Leffler CW, Busija DW, Beasley DG, Armstead WM, Mirro R. Postischemic cerebral microvascular responses to norepinephrine and hypotension in newborn pigs. Stroke 1989; 20: 541-546.

2. Temesvari P, Joo F, Koltai M, et al. Cerebroprotective effect of dexamethasone by increasing the tolerance to hypoxia and preventing brain oedema in newborn piglets with experimental pneumothorax. Neurosci Lett 1984; 49: 87-92.

3. Lorek A, Takei Y, Cady EB, et al. Delayed ("secondary") cerebral energy failure after acute hypoxia-ischemia in the newborn piglet: continuous 48-hour studies by phosphorus magnetic resonance spectroscopy. Pediatr Res 1994; 36: 699-706.

4. Thoresen M, Penrice J, Lorek A, et al. Mild hypothermia after severe transient hypoxia-ischemia ameliorates delayed cerebral energy failure in the newborn piglet. Pediatr Res 1995; 37: 667-670.

5. McCulloch KM, Raju TN, Navale S, et al. Developing a long-term surviving piglet model of neonatal hypoxicischemic encephalopathy. Neurol Res 2005; 27: 16-21.

6. Rootwelt T, Loberg EM, Moen A, Oyasaeter S, Saugstad OD. Hypoxemia and reoxygenation with $21 \%$ or $100 \%$ oxygen in newborn pigs: changes in blood pressure, base deficit, and hypoxanthine and brain morphology. Pediatr Res 1992; 32: 107-113.

7. DiGiacomo JE, Pane CR, Gwiazdowski S, Mishra OP, Delivoria-Papadopoulos M. Effect of graded hypoxia on brain cell membrane injury in newborn piglets. Biol Neonate 1992; 61: 25-32.

8. Foster KA, Colditz PB, Lingwood BE, Burke C, Dunster KR, Roberts MS. An improved survival model of hypoxia/ischaemia in the piglet suitable for neuroprotection studies. Brain Res 2001; 919: 122-131.

9. Domoki F, Olah O, Zimmermann A, et al. Hydrogen is neuroprotective and preserves cerebrovascular reactivity in asphyxiated newborn pigs. Pediatr Res 2010; 68: 387-392.

10. Martin LJ, Brambrink AM, Lehmann C, et al. Hypoxiaischemia causes abnormalities in glutamate transporters and death of astroglia and neurons in newborn striatum. Ann Neurol 1997; 42: 335-348.

11. Ni X, Yang ZJ, Wang B, et al. Early antioxidant treatment and delayed hypothermia after hypoxia-ischemia have no additive neuroprotection in newborn pigs. Anesth Analg 2012; 115: 627-637.

12. Domoki F, Zolei D, Olah O, et al. Evaluation of laserspeckle contrast image analysis techniques in the cortical microcirculation of piglets. Microvasc Res 2012; 83: 311-317.
13. Rojas-Ochoa LF, Lacoste D, Lenke R, Schurtenberger P, Scheffold F. Depolarization of backscattered linearly polarized light. J Opt Soc Am A Opt Image Sci Vis 2004; 21 : 1799-1804.

14. Rice JE, $3^{\text {rd }}$, Vannucci RC, Brierley JB. The influence of immaturity on hypoxic-ischemic brain damage in the rat. Ann Neurol 1981; 9: 131-141.

15. Dragun P, Makarewicz D, Wojcik L, Ziemka-Nalecz M, Slomka M, Zalewska T. Matrix metaloproteinases activity during the evolution of hypoxic-ischemic brain damage in the immature rat. The effect of 1-methylnicotinamide (MNA). J Physiol Pharmacol 2008; 59: 441-455.

16. Kristek F, Malekova M, Ondrias K, Cacanyiova S. Blood pressure-independent hypotrophy of the heart, kidneys and conduit arteries after 7-nitroindazole administration to Wistar rats from the prenatal period to adulthood. $J$ Physiol Pharmacol 2013; 64: 35-39.

17. Thoresen M, Haaland K, Loberg EM, et al. A piglet survival model of posthypoxic encephalopathy. Pediatr Res 1996; 40: 738-748.

18. De Salles AA, Solberg TD, Mischel P, et al. Arteriovenous malformation animal model for radiosurgery: the rete mirabile. AJNR Am J Neuroradiol 1996; 17: 1451-1458.

19. Haaland K, Orderud WJ, Thoresen M. The piglet as a model for cerebral circulation: an angiographic study. Biol Neonate 1995; 68: 75-80.

20. Laptook AR, Stonestreet BS, Oh W. The effect of carotid artery ligation on brain blood flow in newborn piglets. Brain Res 1983; 276: 51-54.

21. Iwata O, Iwata S, Bainbridge A, et al. Supra- and subbaseline phosphocreatine recovery in developing brain after transient hypoxia-ischaemia: relation to baseline energetics, insult severity and outcome. Brain 2008; 131: 2220-2226.

22. Faulkner S, Bainbridge A, Kato T, et al. Xenon augmented hypothermia reduces early lactate/ $\mathrm{N}$-acetylaspartate and cell death in perinatal asphyxia. Ann Neurol 2011; 70: 133-150.

23. Robertson NJ, Faulkner S, Fleiss B, et al. Melatonin augments hypothermic neuroprotection in a perinatal asphyxia model. Brain 2013; 136: 90-105.

24. Robertson NJ, Kato T, Bainbridge A, et al. Methyl-isobutyl amiloride reduces brain Lac/NAA, cell death and microglial activation in a perinatal asphyxia model. $J$ Neurochem 2013; 124: 645-657.

25. Farkas E, Luiten PG, Bari F. Permanent, bilateral common carotid artery occlusion in the rat: a model for chronic cerebral hypoperfusion-related neurodegenerative diseases. Brain Res Rev 2007; 54: 162-180.

26. Sakr HF, Khalil KI, Hussein AM, Zaki MS, Eid RA, Alkhateeb M. Effect of dehydroepiandrosterone (DHEA) on memory and brain derived neurotrophic factor (BDNF) in a rat model of vascular dementia. $J$ Physiol Pharmacol 2014; 65: 41-53.

27. Grant DA, Franzini C, Wild J, Eede KJ, Walker AM. Autoregulation of the cerebral circulation during sleep in newborn lambs. $J$ Physiol 2005; 564: 923-930.

28. Baldwin BA, Bell FR. Blood flow in the carotid and vertebral arteries of the sheep and calf. J Physiol 1963; 167: 448-462.

29. Guan J, Bennet L, George S, et al. Insulin-like growth factor-1 reduces postischemic white matter injury in fetal sheep. J Cereb Blood Flow Metab 2001; 21: 493-502.

30. Laptook A, Stonestreet BS, Oh W. Autoregulation of brain blood flow in the newborn piglet: regional differences in flow reduction during hypotension. Early Hum Dev 1982; 6: 99-107.

31. Munkeby BH, Lyng K, Froen JF, et al. Morphological and hemodynamic magnetic resonance assessment of early 
neonatal brain injury in a piglet model. J Magn Reson Imaging 2004; 20: 8-15.

32. Hou XL, Zhou YX, Zhou CL, Ding HY, Ding HS. Comparison of the models of acute hypoxia and hypoxicischemia in newborn piglets [in Chinese]. Beijing $\mathrm{Da}$ Xue Хиe Bao 2009; 41: 702-706.

33. Domoki F, Zimmermann A, Cserni G, Bori R, Temesvari P, Bari F. Reventilation with room air or $100 \%$ oxygen after asphyxia differentially affects cerebral neuropathology in newborn pigs. Acta Paediatr 2006; 95: 1109-1115.

34. Ni X, Yang ZJ, Carter EL, Martin LJ, Koehler RC. Striatal neuroprotection from neonatal hypoxia-ischemia in piglets by antioxidant treatment with EUK-134 or edaravone. Dev Neurosci 2011; 33: 299-311.

Received: February 10, 2014

Accepted: July 22, 2014

Author's address: Dr. Ferenc Domoki, Department of Physiology, Faculty of Medicine, University of Szeged, 10 Dom ter Street, H-6720 Szeged, Hungary.

E-mail: domoki.ferenc@med.u-szeged.hu 
II 


\title{
MOLECULAR HYDROGEN AFFORDS NEUROPROTECTION IN A TRANSLATIONAL PIGLET MODEL OF HYPOXIC-ISCHEMIC ENCEPHALOPATHY
}

\author{
Department of Physiology, University of Szeged, School of Medicine, Szeged, Hungary
}

\begin{abstract}
Hypoxic-ischemic encephalopathy (HIE) is the major consequence of perinatal asphyxia (PA) in term neonates. Although the newborn piglet is an accepted large animal PA/HIE model, there is no consensus on PA-induction methodology to produce clinically relevant HIE. We aimed to create and to characterize a novel PA model faithfully reproducing all features of asphyxiation including severe hypercapnia resulting in HIE, and to test whether $\mathrm{H}_{2}$ is neuroprotective in this model. Piglets were anaesthetised, artificially ventilated, and intensively monitored (electroencephalography, core temperature, $\mathrm{O}_{2}$ saturation, arterial blood pressure and blood gases). Asphyxia (20 min) was induced by ventilation with a hypoxic-hypercapnic $\left(6 \% \mathrm{O}_{2}-20 \% \mathrm{CO}_{2}\right)$ gas mixture. Asphyxia-induced changes in the cortical microcirculation were assessed with laser-speckle contrast imaging and analysis. Asphyxia was followed by reventilation with air or air containing hydrogen $\left(2.1 \% \mathrm{H}_{2}, 4\right.$ hours). After 24 hours survival, the brains were harvested for neuropathology. Our PA model was characterized by the development of severe hypoxia $\left(\mathrm{pO}_{2}=27 \pm 4 \mathrm{mmHg}\right)$, and combined acidosis $\left(\mathrm{pH}=6.76 \pm 0.04 ; \mathrm{pCO}_{2}=114 \pm 11 \mathrm{mmHg}\right.$; lactate $\left.=12.12 \pm 0.83 \mathrm{mmol} / \mathrm{L}\right)$, however, cortical ischemia did not develop during the stress. Severely depressed electroencephalography (EEG), and marked neuronal injury indicated the development of HIE. $\mathrm{H}_{2}$ was neuroprotective shown both by the enhanced recovery of EEG and by the significant preservation of neurons in the cerebral cortex, hippocampus, basal ganglia, and the thalamus. $\mathrm{H}_{2}$ appeared to reduce oxidative stress shown by attenuation of 8-hydroxy-2'-deoxyguanosine immunostaining. In summary, this new PA piglet model is able to induce moderate/severe HIE, and the efficacy of hydrogen post-treatment to preserve neuronal activity/function in this PA/HIE model suggests the feasibility of this safe and inexpensive approach in the treatment of asphyxiated babies.
\end{abstract}

Key words: hydrogen, neuroprotection, hypoxic-ischemic encephalopathy, laser speckle contrast imaging, perinatal asphyxia, electroencephalography

\section{INTRODUCTION}

Infections and intrapartum-related complications are the leading causes of child mortality under the age of five (responsible for $\sim 6.3$ million deaths annually worldwide), and approximately $40 \%$ of these children die during the neonatal period $(1,2)$. Birth asphyxia is one of the major contributor to early neonatal loss, affecting $3-4$ newborns out of 1000 live births in developing countries, accounting for around 1 million neonatal deaths annually (3). Prolonged deprivation of oxygen around the time of delivery (4) is defined as perinatal asphyxia (PA) and it is presented with hypoxemia, hypercapnia and acidosis (5) eliciting an immediate redistribution of blood flow to the vital organs thus compromising the nutrition of other tissues. If the respiratory distress-induced compensatory mechanisms are exhausted, tissue hypoperfusion may occur, risking permanent hypoxic/ischemic damage. Hypoxic-ischemic encephalopathy (HIE) is one of the most severe consequence of PA with a wide clinical spectrum among survivors: cerebral palsy (motor skill abnormalities), seizure activity, feeding disorders as well as mental- and somatic retardation, vision-, hearing- and speech disorders that constitute a life-long social and financial burden for the health care system and affected families $(6,7)$. Critical care of asphyxiated newborns requires immediate and adequate resuscitation techniques (8) along with effective neuroprotective interventions to prevent the progressive deterioration of the neonatal brain function resulting in HIE. Currently mild systemic hypothermia is the gold standard neuroprotective therapy to alleviate asphyxia-induced brain damage (9-11), but clearly further efforts are needed to establish additional therapeutic approaches that can compliment the existing treatment using animal models that have already been an invaluable aid to study the pathophysiology of PA/HIE (12).

The neuroprotective application of molecular hydrogen was first employed by Ohsawa et al. who reported that hydrogen was a selective hydroxyl radical scavenger and was found neuroprotective in an adult rat stroke model (13). Since then, many preclinical and even clinical studies reported widespread cytoprotective, anti-inflammatory and anti-apoptotic effects of this gas (14). Concerning PA/HIE research, some $(15,16)$ but not all (17) studies found molecular hydrogen to be neuroprotective in rodent PA/HIE models. As far as we know, 
our research group was the first to test the effect of molecular hydrogen in a large animal (newborn piglet) model following asphyxia induced by airway obstruction. We found that reventilation after $8-10 \mathrm{~min}$ asphyxia with a gas mixture containing $2.1 \%$ hydrogen gas prevented both acute (1 hour) and delayed ( 24 hours) neuronal-vascular dysfunction assessed by determination of PA-sensitive cerebrovascular responses (18, 19). Unfortunately, the neuronal damage induced by this stress was relatively mild to be able to fully assess the neuroprotective potential of hydrogen, as electroencephalography (EEG) recovered in 24 hours in virtually all asphyxiated animals and neuropathology revealed also only mild to moderate neuronal injury in the untreated asphyxiated animals too (19). Therefore, we sought to establish a translational moderate/severe PA/HIE piglet model that reproduced all clinical features of PA. Although, the newborn piglet has become an accepted large animal model of the human term neonate, the precise methodology to elicit a clinically feasible experimental PA remains undetermined. Numerous piglet PA models were employed in the past decades, ranging from bilaterally induced pneumothorax (20), suspended ventilation (21) or raised intracranial pressure to produce selective cerebral ischemia (22) offering limited clinical feasibility in terms of human PA pathophysiology. Recently, hypoxic ventilation/reoxygenation followed by airway obstruction (the Johns Hopkins model (23)) or hypoxic ventilation with bilaterally occluded carotid arteries (the University College London model (24)) are extensively applied methods (i.e. $6 \% \mathrm{O}_{2}$ for $30 \mathrm{~min}$. (25)). We have recently shown with laser speckle contrast imaging (LSCI), that bilateral carotid artery occlusion does not reduce cortical blood perfusion in piglets (26) in accordance with the unique anatomy of extracerebral large arteries (27), although, bilateral carotid artery occlusion (28) or 4-vessels occlusion (29) will induce true cerebral ischemia in rats. Furthermore, hypoxic ventilation alone does not induce severe hypercapnia that could critically affect the development of seizures (30) - a common complication in clinical management of PA/HIE.

The purpose of the present study was twofold: 1) to establish and to characterize a clinically relevant and reproducible PA/HIE piglet model that reproduces the human conditions well. To describe the developing asphyxia in detail, we also determined cortical blood flow (CoBF) changes during asphyxia and in the acute reventilation period; 2) to test the putative neuroprotective effect of hydrogen in our severe PA/HIE model. We also performed a meta-analysis on the structural brain damage between the current and our previously reported PA model (19).

\section{MATERIALS AND METHODS}

Animals

Newborn ( $\sim 1$ day old) male Large-White piglets (body weight: $1.5-2.5 \mathrm{~kg}, \mathrm{n}=28)$ were obtained from a local company (Pigmark Ltd., Co., Szeged, Hungary) and delivered to the laboratory on the morning of the experiments. All procedures were approved by the Animal Care and Use Committee of the University of Szeged. Anaesthesia was induced by intraperitoneal injection of sodium thiopental $(45 \mathrm{mg} / \mathrm{kg}$; Sandoz, Kundl, Austria). Piglets were intubated via tracheotomy and artificially ventilated with warmed, humidified medical air $\left(21 \% \mathrm{O}_{2}\right.$, balance $\mathrm{N}_{2}$ ) that could be supplemented with oxygen via a pressure-controlled neonatal ventilator. Respiratory settings (fraction of inspired $\mathrm{O}_{2}\left(\mathrm{FiO}_{2}\right): 0.21-0.25$; respiratory rate $(\mathrm{RR})$ : $30-351 / \mathrm{min}$, peak inspiratory pressure: $120-135$ $\mathrm{mmH}_{2} \mathrm{O}$ ) were adjusted to maintain blood gas values and oxygen saturation in the physiologic range. The right femoral vein was catheterized under aseptic conditions to maintain anaesthesia/analgesia with a bolus injection of morphine (100 $\mu \mathrm{g} / \mathrm{kg}$; Teva, Petach Tikva, Israel) and midazolam $(250 \mu \mathrm{g} / \mathrm{kg}$; Torrex Pharma, Vienna, Austria), then with continuous infusion of morphine $(10 \mu \mathrm{g} / \mathrm{kg} / \mathrm{h})$, midazolam $(250 \mu \mathrm{g} / \mathrm{kg} / \mathrm{h})$ and fluids (5\% glucose, $0.45 \% \mathrm{NaCl} 3-5 \mathrm{ml} / \mathrm{kg} / \mathrm{h}$ ) throughout the whole experiment, as artificial ventilation-induced stress (31) affects the neuro-endocrine system in intubated neonates. A second catheter was placed into the right carotid artery for continuous monitoring of mean arterial blood pressure (MABP) and heart rate (HR). This artery was chosen as ligation of the femoral artery resulted in critical ischemia of the hindlimb over the chosen survival period (personal observations), in contrast, unilateral carotid artery occlusion has been shown to unaffect cerebral blood flow (32). After suturing the surgical incisions, animals were placed into a neonatal incubator (SPC 78-1; Narco Air-Shields, Inc., Hatboro, Pa., USA) in prone position then supplied with electrocardiograph (ECG) and electroencephalograph (Natus Neurology, Middleton, WI, USA) electrodes. Rectal temperature was measured continuously and kept in the physiologic range $\left(38.5 \pm 0.5^{\circ} \mathrm{C}\right)$ with a servocontrolled heating lamp. Oxygen saturation, MABP, HR and ECG were continuously monitored using a Hewlett-Packard M1094 monitor (Palo Alto, California, USA) and recorded online (MecifView, Arlington, Mass., USA). Prophylactic antibiotics were given intravenously (i.v.): penicillin (50 $\mathrm{mg} / \mathrm{kg} / 12 \mathrm{~h}$, Teva, Petah Tikva, Israel) and gentamicin (2.5 $\mathrm{mg} / \mathrm{kg} / 12 \mathrm{~h}$, Sanofi, Paris, France). Seizures were treated with 1 -2 bolus injections of midazolam ( $250 \mu \mathrm{g} / \mathrm{kg}$ ) according to (33). The urinary bladder was tapped by suprapubic puncture at 12 hour of survival. Arterial blood samples ( $300 \mu \mathrm{l} / \mathrm{sample})$ were checked (ABL 5, Radiometer, Denmark or EPOC Blood Analysis, Epocal Inc., Ottawa Canada) at baseline, at the end of asphyxia; then at selected intervals up to 20 hours to keep blood gas values in the physiological range (Fig. 1). After the surgical procedure, one hour recovery period allowed stabilization of monitored physiological parameters prior obtaining baseline values. These parameters were recorded then during PA and for 10 minutes at the beginning of each survival hour.

\section{Electroencephalography $(E E G)$}

EEG activity was recorded via subcutaneously inserted silver pin electrodes above the fronto-parietal and occipital regions. The impedance of all electrodes was checked to be below $5 \mathrm{k} \Omega$. Bandpass filters were applied between $1-70 \mathrm{~Hz}$, and an automatic noise filter (notch filter) rejected the $50 \mathrm{~Hz}$ component. EEG signal was amplified (Nicolet EEG v32, Natus Medical Inc, San Carlos, California, USA), recorded (sampling rate: $250 \mathrm{~Hz}$ ) and visualized online during the entire experiment with the manufacturer's software (Nicolet One). Data were stored on a hard disc of a personal computer. EEG activity was analysed offline with two approaches. First, 10 minute EEG epochs recorded at the beginning of each hour after PA were scored by a researcher unaware of the experimental group using an amplitude-based, incremental scoring system (Table 1), according to (34). Briefly, continuous and high amplitude background activities $(>10 \mu \mathrm{V})$ were given lower scores while severely depressed and isoelectric activities $(<10 \mu \mathrm{V})$ received higher ones. In addition, if seizures appeared in the evaluated hour, 2 extra points were added. Second, EEG power spectrum analysis of the same EEG epochs was performed by the NicoletOne Review software using Fast Fourier Transformation (FFT, linear detrending, Hamming window; $\delta 1-4 \mathrm{~Hz}, \theta 4-8$ $\mathrm{Hz}, \alpha 8-12 \mathrm{~Hz}, \beta 12-30 \mathrm{~Hz}$ ) and absolute band powers were calculated. Total EEG power $\left(\mu \mathrm{V}^{2}\right)$ (summation of bands) values were selected to quantitatively characterize the recovery of brain 
electrical activity following PA and to complement the semiquantitative and the more observer-dependent scoring system.

\section{Experimental perinatal asphyxia (PA)}

After obtaining the baseline physiologic parameters, animals were divided into 3 groups (Fig. 1): time control group (CTR, $\mathrm{n}=$ 7), asphyxia group (ASPH, $n=8$ ) and asphyxia + hydrogen treated group (ASPH $+\mathrm{H}_{2}, \mathrm{n}=8$ ). Animals were randomized between groups ASPH and ASPH $+\mathrm{H}_{2}$ by coin flip. PA in groups ASPH and ASPH $+\mathrm{H}_{2}$ was induced by switching ventilation from medical air to a hypoxic-hypercapnic gas mixture $\left(6 \% \mathrm{O}_{2}, 20 \% \mathrm{CO}_{2}\right.$, balance $\mathrm{N}_{2}$ ) for 20 minutes, reducing the RR to $151 /$ min and stopping the fluid/glucose administration. Piglets were reventilated (RR: 30 $1 / \mathrm{min}$ ) in group ASPH with medical air, whereas in group ASPH + $\mathrm{H}_{2}$ with a gas mixture containing hydrogen gas $\left(2.1 \% \mathrm{H}_{2}, 21 \% \mathrm{O}_{2}\right.$, balance $\mathrm{N}_{2}$ ). In group ASPH $+\mathrm{H}_{2}$, after 4 hours hydrogen treatment was stopped and ventilation with medical air was resumed. An additional group of animals (group LSCI, $n=5$ ) was added to assess CoBF changes during asphyxic conditions. Animals received similar anaesthesia and instrumentation regimen as the other groups then 20 minute asphyxia was induced as in groups ASPH and ASPH $+\mathrm{H}_{2}$ followed by room-air reventilation and short-term survival. Details of LSCI methodology are given in the respective section below.

\section{Histology}

Twenty-four hours after the end of asphyxia, the brains were perfused with cold $\left(4^{\circ} \mathrm{C}\right)$ physiologic saline through the catheterized common carotid arteries. Brains were gently collected and were immersion-fixed in $4^{\circ} \mathrm{C}, 4 \%$ paraformaldehyde solution and further processed after two weeks. Paraffin embedded, $4 \mu \mathrm{m}$ sections were made from the frontal, temporal, parietal, occipital lobes. The haematoxylin-eosin stained slides were evaluated by a researcher blinded to the experimental groups with light microscopy (Leica Microsystems, Wetzlar, Germany). Damaged neurons were identified using the major hallmarks of dark eosinophilic cytosol, as well as pyknotic or disrupted nuclei. The degree of cerebrocortical neuronal damage was determined adapting a previously published scoring system (Table 2) (35) that allowed more comprehensive study of these large brain regions compared to the cell counting used in our previous studies $(18,19)$. Briefly, the pattern of neuronal injury (none $<$ scattered $<$ grouped $<$ panlaminar) was determined in 20 - 20 non-overlapping fields of vision under $20 \times$ magnification in each assessed cortical region. Then, scores $(0-9)$ were given to each region based on the

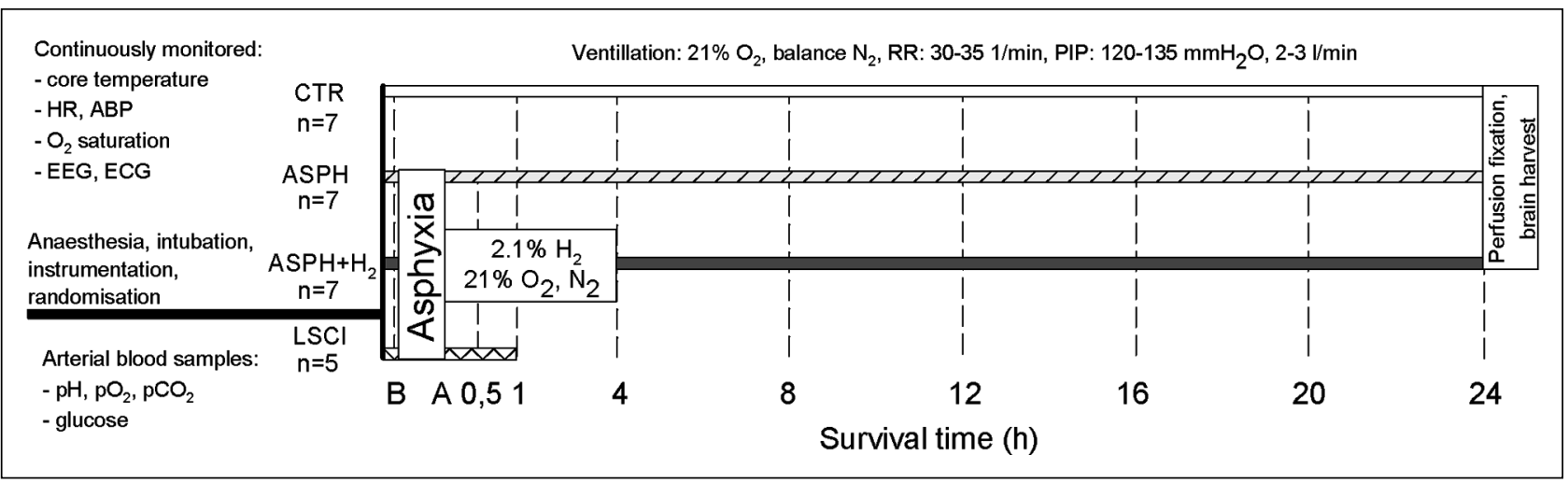

Fig. 1. Experimental protocol and groups. The putative neuroprotective effect of molecular hydrogen $\left(\mathrm{H}_{2}\right)$ against asphyxia-induced hypoxic-ischemic encephalopathy was assessed in 3 groups. After instrumentation and recording of baseline (B) physiological parameters (core temperature, heart rate (HR), arterial blood pressure (ABP) and oxygen saturation as well as ECG and EEG), animals were divided into the following experimental groups: piglets in group CTR $(n=7$, white) served as normoxic time controls. Piglets in groups ASPH $\left(n=7\right.$, light grey, hatched) and $\mathrm{ASPH}+\mathrm{H}_{2}(\mathrm{n}=7$, dark grey) were subjected to 20 min asphyxia (ventilation with $6 \%$ $\mathrm{O}_{2}, 20 \% \mathrm{CO}_{2}$, balance $\mathrm{N}_{2}$; with a respiratory rate (RR) reduced from $30-35$ to $151 / \mathrm{min}$ ). Animals in group ASPH were reventilated with medical air whereas in group ASPH $+\mathrm{H}_{2}$ by a $\mathrm{H}_{2}$-containing gas mixture $\left(2.1 \% \mathrm{H}_{2}, 21 \% \mathrm{O}_{2}\right.$, balance $\left.\mathrm{N}_{2}\right)$. Ventilation in group ASPH $+\mathrm{H}_{2}$ was switched back to medical air at 4 hour of survival. The brains were collected for neuropathology analysis at 24 hour of survival. Piglets in group LSCI $(n=5$, white, crosshatched) were equipped with a closed cranial window to determine the acute effect of asphyxia on the cortical microcirculation using laser speckle contrast imaging (LSCI).

Table 1. Amplitude-based scoring system was applied to visually assess brain electric activity during experimental asphyxia and the evolution of HIE. High amplitude patterns $(>10 \mu \mathrm{V})$ during the initial 10 minute of given time points received lower $(1-3)$ scores, while severely depressed $(<10 \mu \mathrm{V})$ brain electric activity was given higher $(4-7)$ ones. The presence of seizure activity during the evaluated period was indicated by adding 2 extra points to the assessed background activity.

\begin{tabular}{|c|c|}
\hline Score & Amplitude based EEG pattern \\
\hline 1 & $>25 \mu \mathrm{V}$ dominating pattern \\
\hline 2 & $>25 \mu \mathrm{V}$ with short durations $(1-5 \mathrm{~s})$ below \\
\hline 3 & $25-10 \mu \mathrm{V}$ \\
\hline 4 & $10-15 \mu \mathrm{V}$ \\
\hline 5 & Low voltage $(<5 \mu \mathrm{V})$ with bursts $(>25 \mu \mathrm{V}, 1-5 \mathrm{~s})$ \\
\hline 6 & $<5 \mu \mathrm{V}$ \\
\hline 7 & practically isoelectric \\
\hline+2 & electro/clinical convulsion \\
\hline
\end{tabular}


frequency ( $\%$ of 20 examined fields) of the most severe pattern of injury observed. The neuronal damage in the ganglionic cell layer of the cerebellum, basal ganglia, thalamus and the hippocampal $\mathrm{CA} 1$ and CA 3 regions was assessed with cell counting in nonoverlapping areas (in 10, 5, 5, 3, 3 fields of vision respectively; under 20x magnification) as in (19). The impact of asphyxia on cerebellum and subcortical brain regions was expressed as the percentage of damaged neurons. In order to be able to compare the severity of asphyxia-induced cortical neuronal damage at 24 hour of survival between the present new and our previously published (19) asphyxia method (20 min ventilation with $6 \% \mathrm{O}_{2} / 20 \% \mathrm{CO}_{2}$ versus 8 min suspension of ventilation), we re-analyzed the cortical samples from the ASPH group $(n=9)$ of the previous study using the scoring system as well, and we present the combined neuropathology scores in the Results for this group as well.

\section{8-hydroxy-2'-deoxyguanosine (8-OHdG) immunohistochemistry}

Parietal cortex tissue microarrays were produced using a custom-made stainless steel tissue puncher $(3 \mathrm{~mm})$ from the paraffin tissue blocks, sectioned at $4 \mu \mathrm{m}$, mounted on sylanized slides and processed for 8-OHdG immunohistochemistry using a LEICA BOND-MAX automated immunostainer (Leica Microsystems). In the immunostainer, the slides were dewaxed at $72^{\circ} \mathrm{C}$, and antigen retrieval was performed at $\mathrm{pH}=6$. Then, slides were incubated with a 1:200 dilution of mouse monoclonal primary antibody against $8-\mathrm{OHdG}$ (JaICA Inc., Fukuroi, Japan) for $20 \mathrm{~min}$ followed by horseradish peroxidase-conjugated rabbit anti-mouse secondary antibody for 15 minutes. 3,3'diaminobenzidine was used to visualize the immunostaining, then the slides were counterstained with haematoxylin, to visualize the cell nuclei. The slides were covered with a coverslip then scanned in a slide scanner (Pannoramic MIDI, 3DHISTECH Ltd., Budapest, Hungary), and visualized on a personal computer using the Pannoramic Viewer software (3DHISTECH Ltd.) at 40x magnification. Homogenous, strong nuclear $8-\mathrm{OHdG}$ immunoreactivity was considered as a sign of oxidative damage and the ratio of such nuclei to the total number of cell nuclei was determined and presented in the Results.

\section{Neuron specific enolase (NSE) ELISA}

One $\mathrm{ml}$ arterial blood samples were taken at regular intervals (baseline, 4 and 20 hour of survival) into EDTA-coated microcentrifuge tubes supplemented with $40 \mu$ protease inhibitor cocktail procured following the manufacturer's directions (cOmplete, EDTA-free Protease Inhibitor Cocktail, Roche Diagnostics Gmbh, Mannheim, Germany). The blood samples were centrifuged at $2200 \mathrm{~g}$ and $4^{\circ} \mathrm{C}$ for 5 minutes (Model 5418R, Eppendorf, Hamburg, Germany) and the plasma was transferred to fresh microcentrifuge tubes and stored at $-80^{\circ} \mathrm{C}$. Blood plasma NSE levels were determined using a commercially available, porcine-specific sandwich ELISA kit (Elabscience Biotechnology Co., Ltd., Wuhan, China). The measurements were performed in duplicates following the manufacturer's instructions using a microplate reader (Fluostar Optima, BMG LABTECH, Gmbh, Ortenberg, Germany) at $\lambda=450 \mathrm{~nm}$.

\section{Laser-speckle contrast imaging and analysis (LSCI/LASCA) to study cortical blood flow (CoBF) changes}

CoBF changes induced by PA were recorded ( 5 min baseline, $20 \mathrm{~min}$ PA, $10 \mathrm{~min}$ room air reventilation) and assessed by LSCI/LASCA in additional animals (Fig. 1, group LSCI, $\mathrm{n}=5$ ). Our custom-designed speckle imager and the LASCA software has been described recently in details (26). Briefly, LSCI/LASCA allows to monitor rapid changes in tissue perfusion by determining the autocorrelation decay time $(\tau)$ of interference patterns produced by laser light (laser speckle) scattered from moving particles (red blood cells). The average velocity is directly proportional to $1 / \tau$. Anaesthesia and initial surgical procedures were similar to the other groups, however, in these animals after a circular craniotomy and removal of the dura mater, a closed cranial window with 3 injectible ports was inserted over the left parietal region as described in (36). The cranial window was sealed with bone wax, cyanoacrylate and dental acrylate (Lang Dental Manufacturing Co Inc, Wheeling, IL, USA). After $45 \mathrm{~min}$ incubation, the subarachnoidal space under the cranial window $(\sim 3 \mathrm{ml}$ chamber volume) was filled with warmed, $\mathrm{pH}$ equilibrated artificial cerebrospinal fluid (aCSF) through the ports. The cranial window was illuminated with a near-infrared diode laser $(\lambda=808 \mathrm{~nm})$ and speckle images were recorded $(1 \mathrm{~Hz}, 2 \mathrm{~ms})$ through an operating microscope by a PL-B771F monochrome camera (PixeLINK, Ottawa, Canada), visualised online with a custom-made software and stored on a personal computer. LASCA was performed offline; regions of interests (4 for each timepoint/animal) were selected over the cortical parenchyma not obstructed by surface pial vessels. The determined $1 / \tau$ values were normalized for baseline, and data were then expressed as relative changes from baseline.

\section{Statistical analysis}

Results were analysed offline and plotted using SigmaPlot (v12.0, Systat Software Inc., San Jose, CA., USA) core temperature, saturation, $\mathrm{HR}$ and MABP as well as arterial blood gas and metabolic parameters, cell counts, relative CoBF changes

Table 2. Cortical neuronal damage was assessed using a neuropathology scoring system according to Foster et al. (35) based on the occurrence of the most severe pattern observed in 20 visual fields/regions. Higher scores represent more severe neuronal damage.

\begin{tabular}{|c|c|c|}
\hline \multirow{2}{*}{ Score } & Morphology of cortical damage & Ratio of the most severe pattern per area \\
\hline 0 & \multicolumn{2}{|c|}{ No damage } \\
\cline { 2 - 3 } 2 & Scattered & $<20 \%$ \\
3 & & $21-50 \%$ \\
4 & Grouped & $>50 \%$ \\
5 & & $<20 \%$ \\
6 & & $21-50 \%$ \\
7 & Panlaminar & $>50 \%$ \\
8 & & $<20 \%$ \\
9 & & $21-50 \%$ \\
\cline { 2 - 3 } & & $>50 \%$ \\
\hline
\end{tabular}


were expressed as mean \pm S.E.M. EEG scores, EEG total power and neuropathology scores were expressed as median, $25-75$ and $5-95$ percentiles. Normality was tested with the ShapiroWilk test. Parametric data were compared with one-way- or twoway repeated measure of analysis of variance (RM ANOVA) followed by the Student-Newman-Keuls post hoc test. For nonparametric data RM ANOVA on ranks and for pairwise comparisons the Student-Newman-Keuls post hoc test was applied. The significance of linear regression coefficients was tested with ANOVA. Level of significance $(\mathrm{P})$ was set at 0.05 .

\section{RESULTS}

Characterization of the perinatal asphyxia stress

The normoxic time control animals (CTR) had physiological core temperature, MABP, HR, oxygen saturation, arterial $\mathrm{pH}$, blood gas values (37), and glucose levels (Fig. 2) as well as continuous EEG activity throughout the experiments.

In groups ASPH and ASPH $+\mathrm{H}_{2}$, baseline MABP, HR, oxygen saturation, arterial $\mathrm{pH}, \mathrm{pCO}_{2}, \mathrm{pO}_{2}$ and glucose values

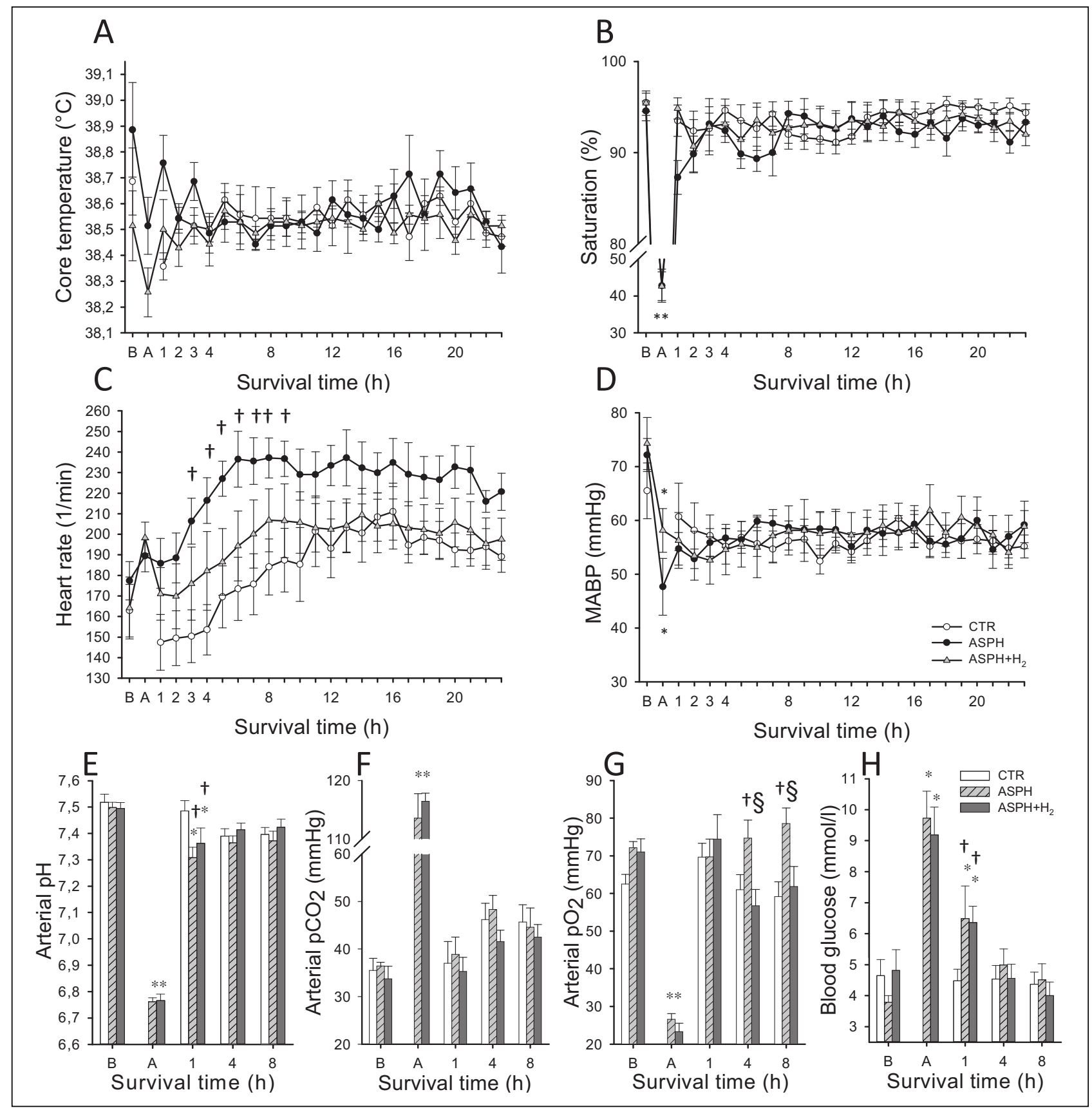

Fig. 2. Monitored physiological parameters in group CTR $(n=7)$, group ASPH $(n=7)$ and group ASPH $+\mathrm{H}_{2}(n=7)$ during baseline $(B)$, in the last minute of $20 \mathrm{~min}$ asphyxia (A) and the survival period: core temperature (Panel A), oxygen saturation (Panel B), heart rate (Panel $\mathrm{C}$ ), and mean arterial blood pressure (MABP) (Panel D). On the bar graphs arterial blood pH (Panel E), partial pressures of carbon dioxide (Panel F) and oxygen (Panel G), as well as glucose levels (Panel H) are presented only up to 8 hours of reventilation as there was no difference among the experimental groups at later time points and they remained in the physiological range. Asphyxia induced similar degree of acidosis, hypercapnia and hypoxia in groups ASPH and ASPH $+\mathrm{H}_{2}$. The asphyxiated groups did not differ markedly in the monitored parameters during the survival period except that heart rate was significantly higher in group ASPH during the first $10-12$ hours of survival (Panel C). Data are shown as means \pm S.E.M. $\mathrm{P}<0.05$. ${ }^{*}$ versus baseline, $\dagger$ versus time control group and $\S$ versus ASPH $+\mathrm{H}_{2}$ at given time point. 
were all similar to the time controls (Fig. 2). After the onset of asphyxia, however, MABP and HR were markedly raised as oxygen saturation rapidly fell (Fig. 3). The severe reduction in oxygen saturation in addition to the values obtained by pulsoxymetry were confirmed by blood gas analysis, in the arterial blood sample taken at the end of PA, this central oxygen saturation also fell from $94 \pm 5$ to $13 \pm 4 \%$ (data from group LSCI). The EEG became isoelectric within $1-2$ min after the onset of PA. In additional animals (group LSCI) using LSCI/LASCA, we found that this stress resulted in a modest $(\sim 30-50 \%)$ increase in CoBF (Fig. 4). Reventilation resulted in quick restoration of oxygen saturation with a simultaneous further increase in MABP and HR that was gradually restored (Fig. 3). CoBF increased also further thus showing a strong reactive hyperaemic response (Fig. 4).

Asphyxia resulted in severe acidosis, hypercapnia and hypoxemia along with hyperglycemia that were similar in groups ASPH and ASPH $+\mathrm{H}_{2}$ (Fig. 2). In addition to the hypercapnia-induced respiratory acidosis, marked metabolic component of the acidosis was also demonstrated as blood lactate levels were profoundly elevated from $1.80 \pm 0.55$ to $12.12 \pm 0.83 \mathrm{mmol} / \mathrm{l}$. Blood lactate was moderately elevated at 1 hour $(5.28 \pm 1.06)$ but returned to baseline $(1.33 \pm 0.10)$ by 4 hour of reventilation (group ASPH, $n=7$ ).
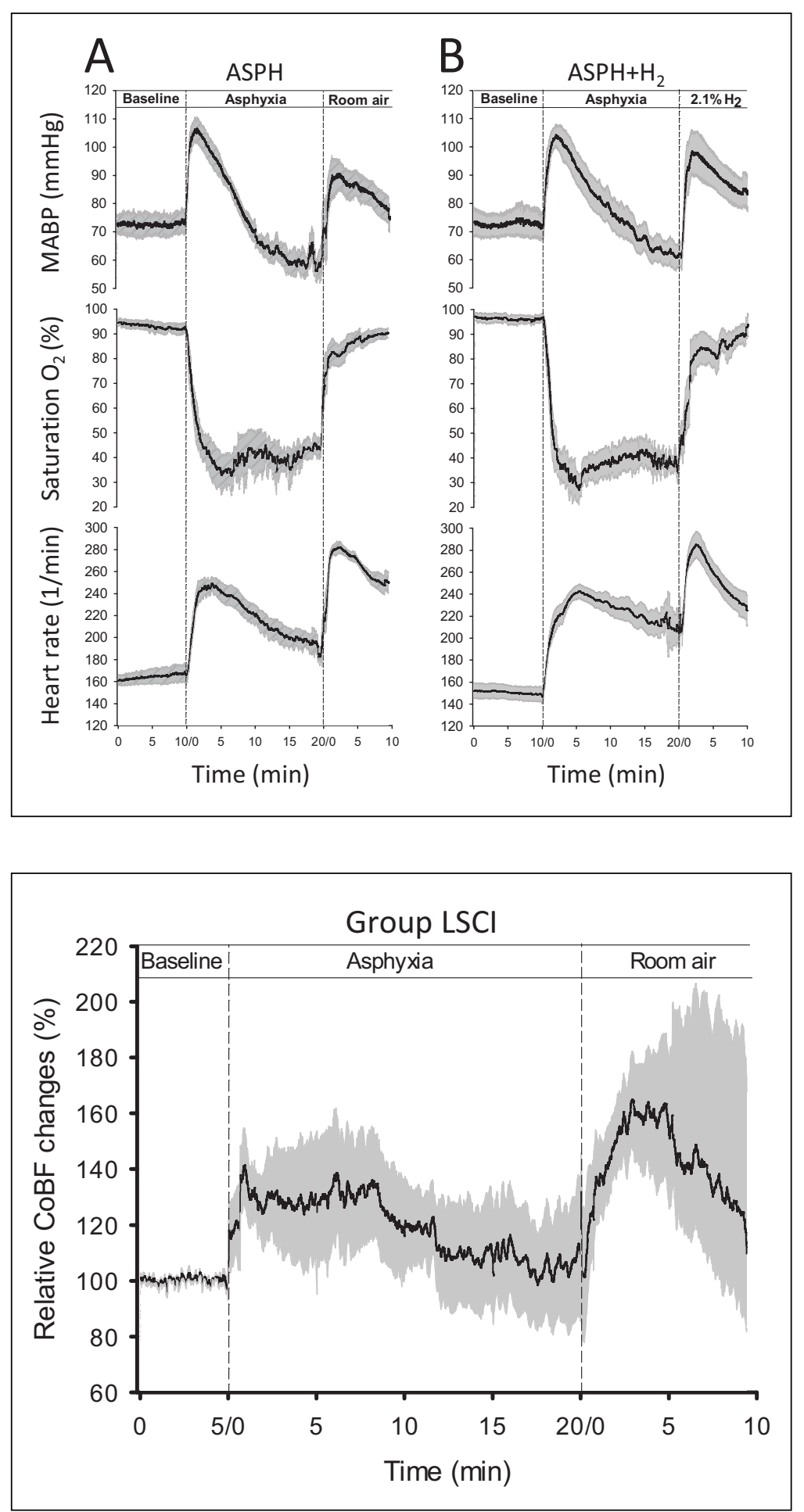

Fig. 3. Hemodynamic response to asphyxia in groups ASPH and ASPH $+\mathrm{H}_{2}$. Asphyxia induced a biphasic response with an acute increases in mean arterial blood pressure (MABP) (top panel), and heart rate (HR) (bottom panel) as oxygen saturation (middle panel) fell indicating the onset of hypoxia. This initial increase in MABP was however not maintained, beyond $10 \mathrm{~min}$ moderate MABP reduction was recorded. Reventilation resulted again in a marked increase in both MABP and HR that were gradually returning towards baseline values. The hemodynamic response was nearly identical in group ASPH $(n=7$; Panel A) and group ASPH $+\mathrm{H}_{2}(\mathrm{n}=7$; Panel $\mathrm{B})$ indicating that hemodynamic consequences of asphyxia were similar in both groups and that hydrogen did not affect hemodynamics in the early reventilation period. Data are presented as means \pm S.E.M. (black lines and the gray shaded areas, respectively).
Fig. 4. Cortical blood flow (CoBF) response to asphyxia/reventilation. Laser Speckle Contrast Imaging and Analysis in group LSCI $(n=5)$ revealed that asphyxia elicited somewhat variable, but always moderate increases in CoBF, hypoperfusion or cortical ischemia did not develop during the stress. However, reventilation resulted in further $\mathrm{CoBF}$ increases representing marked reactive hyperaemia paralleling the systemic hemodynamic response. Data are presented as mean \pm S.E.M. (black line and the grey shaded areas, respectively). 


\section{Hypoxic-ischemic encephalopathy development}

Mortality of PA was $12.5 \%(2 / 16)$ as $1-1$ piglet in groups ASPH and ASPH $+\mathrm{H}_{2}$ were lost at 8 and 12 hours of survival, respectively, due to cardiorespiratory failure. The data from these animals were excluded from the study.
After PA, in groups ASPH and ASPH $+\mathrm{H}_{2}$, normocapnia was restored by 30 minutes after the onset of reventilation $\left(\mathrm{PaCO}_{2}: 39 \pm 2 \mathrm{mmHg}\right.$ and $39 \pm 4 \mathrm{mmHg}$, respectively) and core temperature, MABP, oxygen saturation as well as arterial blood gas and glucose values were maintained in the respective physiological ranges and were statistically not different from the

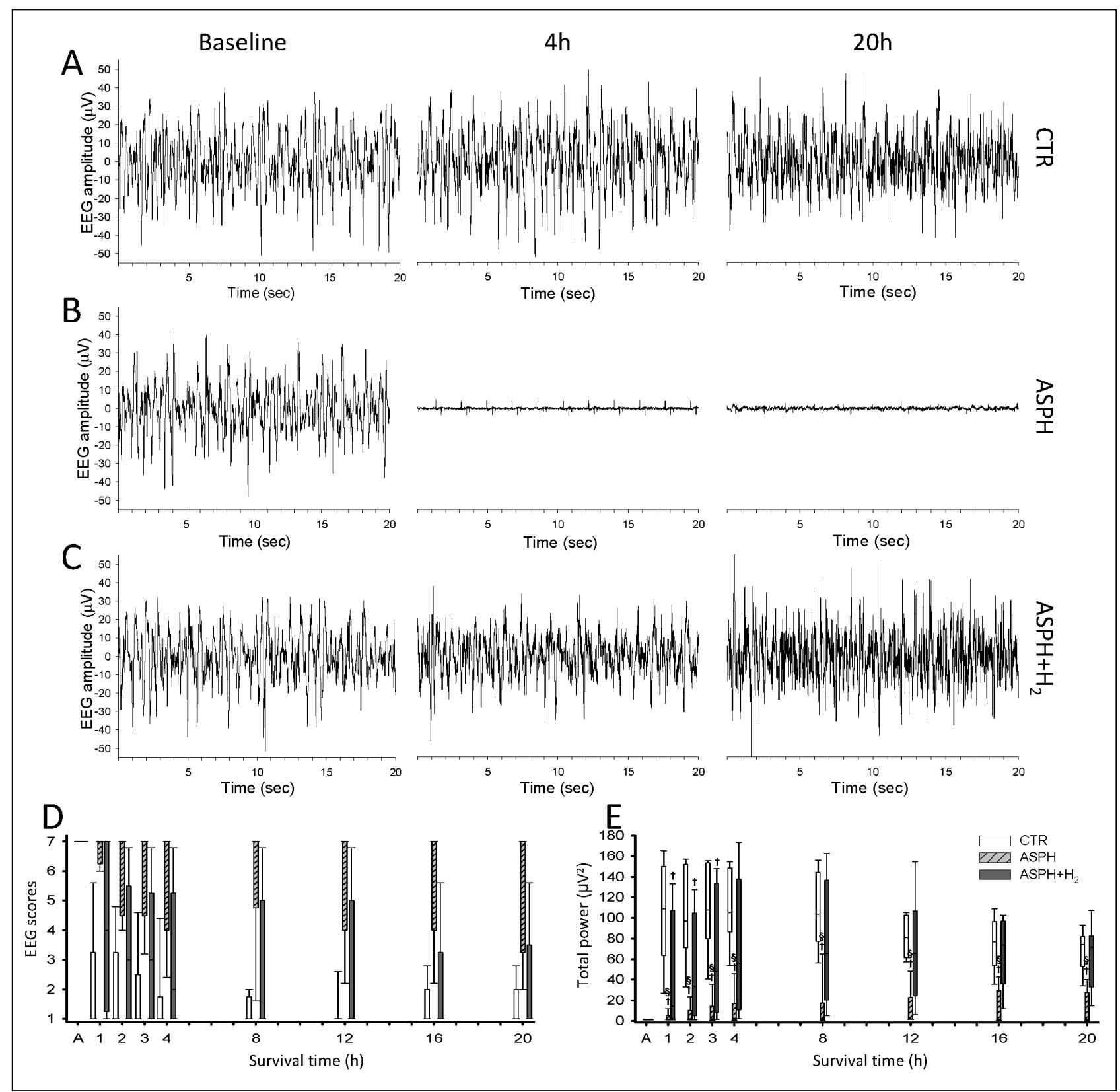

Fig. 5. Representative EEG tracings (occipital electrode, 20 - 20 second epochs displayed from the analyzed $10-10$ minutes tracings at the indicated time points) registered in animals representing the median EEG score values of each group on Panels A, B and C. Similar continuous EEG activity $(>25 \mu \mathrm{V})$ can be observed at baseline in all subjects. The control animal from group CTR (Panel A) maintained high amplitudes throughout the observation period meanwhile 20 minute asphyxia in the piglet from group ASPH (Panel B) elicited severe electrocortical depression. Interestingly, ECG artefacts became prominent over the isoelectric EEG background pattern in this severely injured animal. Molecular hydrogen resulted in restoration of the EEG by 4 hours in this piglet from group ASPH $+\mathrm{H}_{2}(\mathrm{Panel} \mathrm{C)}$. The EEG recordings were analyzed using two methods. First, a custom-made amplitude-based scoring system was created (Table 1), where higher scores represent progressive deterioration of electrical activity (Panel D). Second, total EEG power (Panel E) was determined. EEG tracings of time control animals $(n=7$, white) were characterized by continuous EEG activity and high total power during the entire experiment. Asphyxia (A on Panels D, E), induced isoelectric EEG that was followed by slow regeneration of electrical activity. Regeneration of EEG was more rapid and more complete in the hydrogen-reventilated animals of group ASPH $+\mathrm{H}_{2}(\mathrm{n}=7$, dark grey) shown by lower EEG scores and higher EEG power, as compared to those of group ASPH ( $=7$, light grey, hatched) that were reventilated with air only. Data of Panels D and E are shown as median, $25^{\text {th }}-75^{\text {th }}$, and $5^{\text {th }}-95^{\text {th }}$ percentiles (line, box, and error bars, respectively). $\mathrm{P}<0.05$. $\dagger$ versus time control group at respective time point, $\S$ versus ASPH $+\mathrm{H}_{2}$ at respective time point. 
time control group during the survival period (Fig. 2). Only HR was significantly elevated in group ASPH compared to time controls during the early reventilation (Fig. 2), but significant differences of the monitored physiological parameters between the asphyxiated groups (groups ASPH and ASPH $+\mathrm{H}_{2}$ ) were not observed even then. Some piglets ( 5 versus 7 versus 4 animals in groups CTR, ASPH and ASPH $+\mathrm{H}_{2}$, respectively), required higher $\mathrm{FiO}_{2}$ to maintain similar oxygen saturation that was responsible for the difference in $\mathrm{PaO}_{2}$ during the early reventilation period (Fig. $2 G$ ). Brain electrical activity remained virtually isoelectric in the ASPH group that resulted in low total power and high EEG scores (Fig. 5). Two animals in group ASPH manifested electro-clinical convulsions appearing at 9 and 13 hours of survival, respectively. Seizures were identified by repetitive trunk/limb muscle contractions and characteristic
EEG patterns. Hydrogen administration facilitated the restoration of the EEG activity and most of the animals (5 out of 7) presented continuous background activity by the end of the survival period with higher power values (Fig. 5), furthermore, none of the animals in group ASPH $+\mathrm{H}_{2}$ showed electrographic seizure activity. PA did not induce elevations in plasma NSE levels that were $96.2 \pm 6.7 \%$ and $106.1 \pm 36 \%$ of baseline levels at 4 and 20 hour of survival, respectively (group ASPH, $n=6$ ).

\section{Neuropathology}

In the normoxic time control group, no significant neuronal damage was noted except for the low-frequency occurrence of scattered damaged neurons. In contrast, neuropathology analysis revealed marked cortical neuronal damage in group ASPH that is

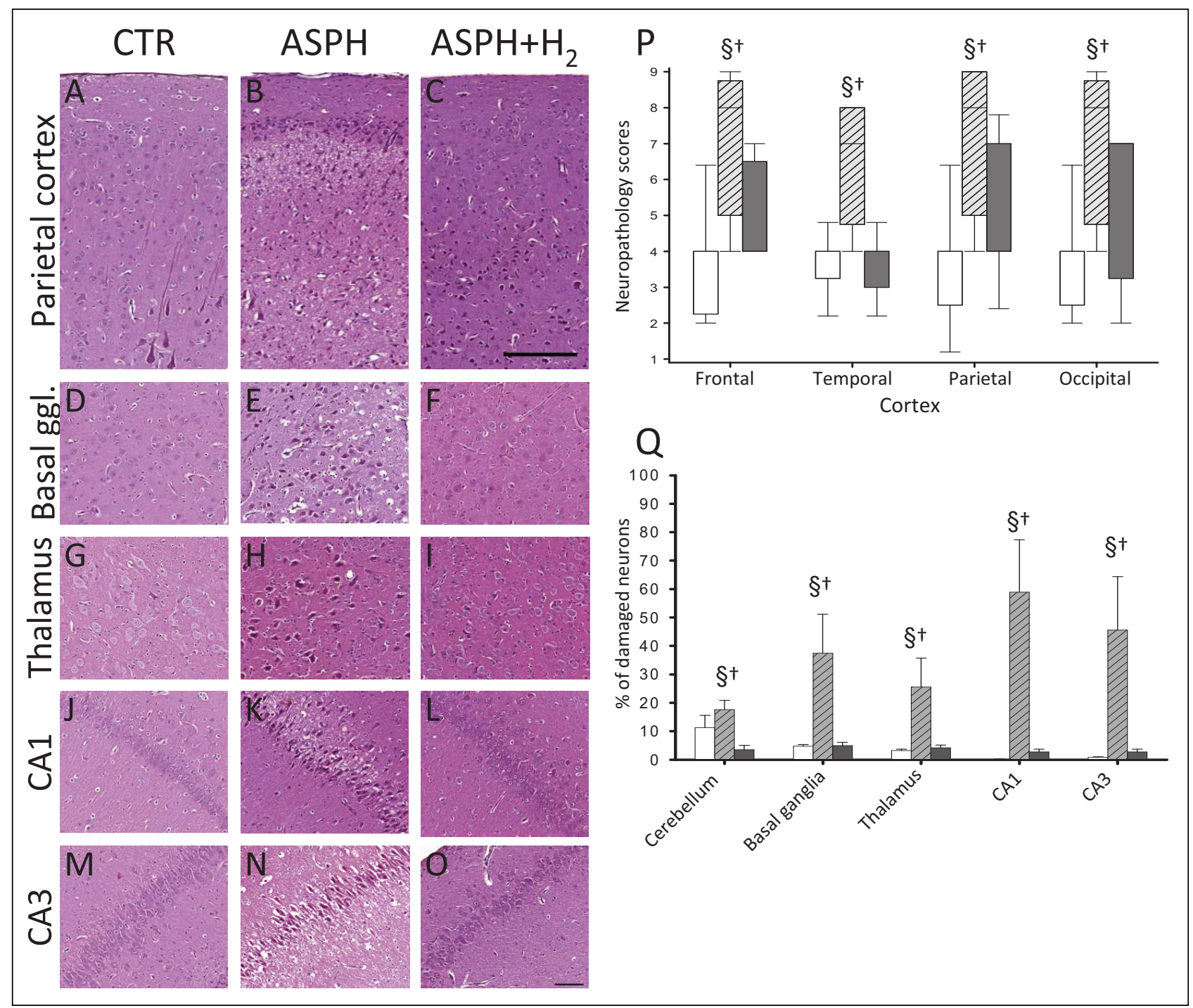

Fig. 6. Histology results and representative photomicrographs taken from subjects representing the median of the groups. Neuronal damage induced by PA in group ASPH and neuroprotection afforded by $\mathrm{H}_{2}$ may be observed in the selected cortical and subcortical areas. Scale bar for photomicrographs A - C: $200 \mu \mathrm{m}$ (top bar), for D - O: $100 \mu \mathrm{m}$ (bottom bar). In all assessed cortical regions (Panel $\mathrm{P}$ ), the hydrogen-reventilated animals (ASPH $+\mathrm{H}_{2}, \mathrm{n}=7$, dark grey) had significantly smaller cortical damage as compared to airreventilated (ASPH, $\mathrm{n}=7$, light grey, hatched) piglets. Brain damage was minimal in the time control animals (CTR, $\mathrm{n}=7$, white). In other brain regions, including the ganglionic cell layer of the cerebellar cortex; the basal ganglia, the thalamus and the CA1 and CA3 regions of the hippocampus (Panel Q) the neuronal damage was determined by cell counting. Similar to the cortex there was a striking neuroprotective effect afforded by hydrogen, especially marked neuroprotection was observed in the hippocampus and the basal ganglia. Data are shown as median, $25^{\text {th }}-75^{\text {th }}$, and $5^{\text {th }}-95^{\text {th }}$ percentiles (line, box, and error bars, respectively for Panel P, and as mean \pm S.E.M. for Panel Q. $\mathrm{P}<0.05$. $\dagger$ versus group CTR and $\S$ versus group ASPH $+\mathrm{H}_{2}$. 


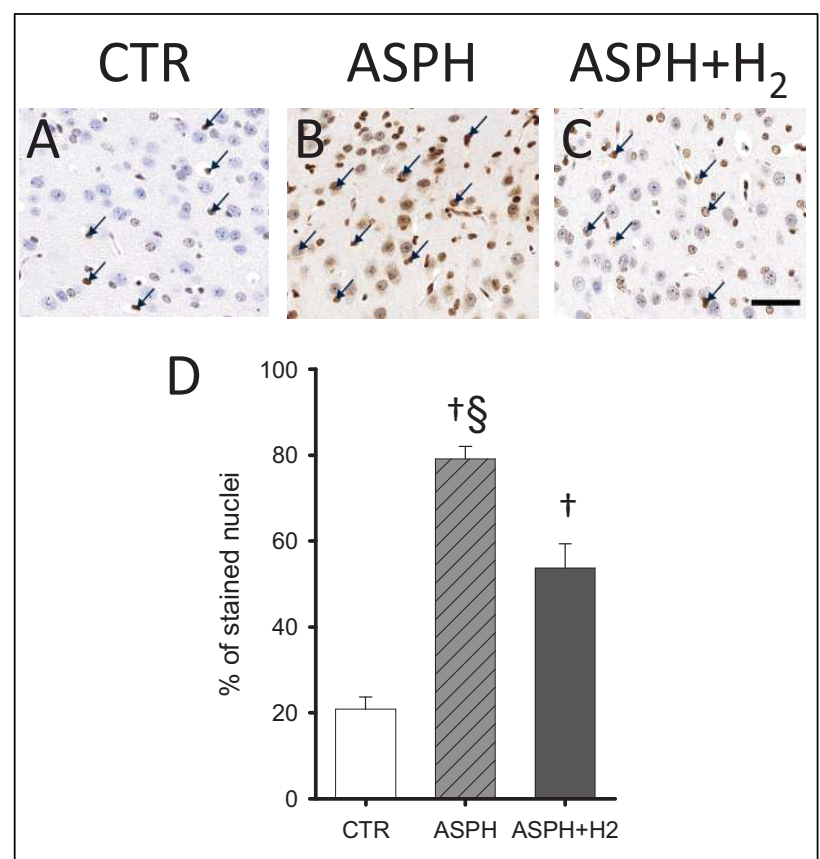

Fig. 7. Hydrogen reduces oxidative stress after PA. Representative photomicrographs (Panels A - C) of 8-OHdG immunopositive nuclei from the parietal cortex. Time control animals (group CTR) exhibited only few strongly stained immunopositive nuclei (arrows). 20 minute asphyxia markedly increased DNA oxidation, resulted in strong immunoreactivity against 8-OHdG at 24 hour of survival (group ASPH). Molecular hydrogen (group ASPH $+\mathrm{H}_{2}$ ) significantly reduced oxidative damage after PA. (Panel D). P < 0.05 . $\dagger$ versus group CTR and $\S$ versus group ASPH $+\mathrm{H}_{2}, \mathrm{n}=14$ -28 regions of interests/ group, scale bar $100 \mu \mathrm{m}$.

reflected by high damage scores (Fig. 6) corresponding to laminar or even confluent panlaminar necrotic lesions virtually in all cortical areas observed at least in some of the assessed fields of view. We compared the cortical neuronal damage induced by our new PA method to our previous ( 8 min tracheal tube occlusion) PA model, by determining also the neuropathology scores of the same cortical regions obtained in our previous study (19). In summary, we found that in group ASPH, the summated damage scores of the four assessed cortical regions were $32 ; 20 ; 36$ (median; $25^{\text {th }} ; 75^{\text {th }}$ percentile), whereas in our previous PA model the values were significantly lower: $17 ; 10 ; 21(\mathrm{P}=0.026)$ indicating a much more robust cerebrocortical damage in the present model. Accordingly, very severe neuronal loss and destructed neuropil were found in the CA1 and CA3 hippocampal regions in group ASPH of the present study, and significant neuronal loss was also obvious in the basal ganglia, the thalamus and the cerebellar Purkinje cells (Fig. 6). Molecular $\mathrm{H}_{2}$ applied in the early reventilation (group ASPH $+\mathrm{H}_{2}$ ) significantly improved neuronal viability in virtually all cortical and subcortical regions studied. Importantly, the hippocampal CA1 and CA3 subfields preserved their structural integrity and most of its cells. Similar preservation of viable neurons was noted in the cerebellum, thalamus and the basal ganglia in the hydrogen treated group $\mathrm{ASPH}+\mathrm{H}_{2}$ (Fig. 6).

In the parietal cortex, the number of $8-\mathrm{OHdG}$ immunoreactive cell nuclei was low in group CTR, however, strong, significantly elevated staining was characteristic of group ASPH and ASPH $+\mathrm{H}_{2}$. However, there was also a significant difference between the ratios of 8-OHdG immunoreactive nuclei between group $\mathrm{ASPH}$ and $\mathrm{ASPH}+\mathrm{H}_{2}$, indicating reduced oxidative stress in the hydrogen treated group (Fig. 7).

\section{DISCUSSION}

In the present study, we introduce a novel piglet PA/HIE model that is relatively simple to induce but still faithfully reproduces all major symptoms associated with PA (isoelectric EEG, severe hypoxemia, hypercapnia, lactacidemia) and elicits significant encephalopathy shown by severely depressed EEG activity and neuronal damage at 24 hours survival. By characterizing our model we showed that this level of asphyxia did not induce severe cerebral ischemia during the PA stress but was able to induce more severe brain damage as compared to our previous PA model (8 min tracheal occlusion) (19). This more robust model thus enabled us to discover the marked
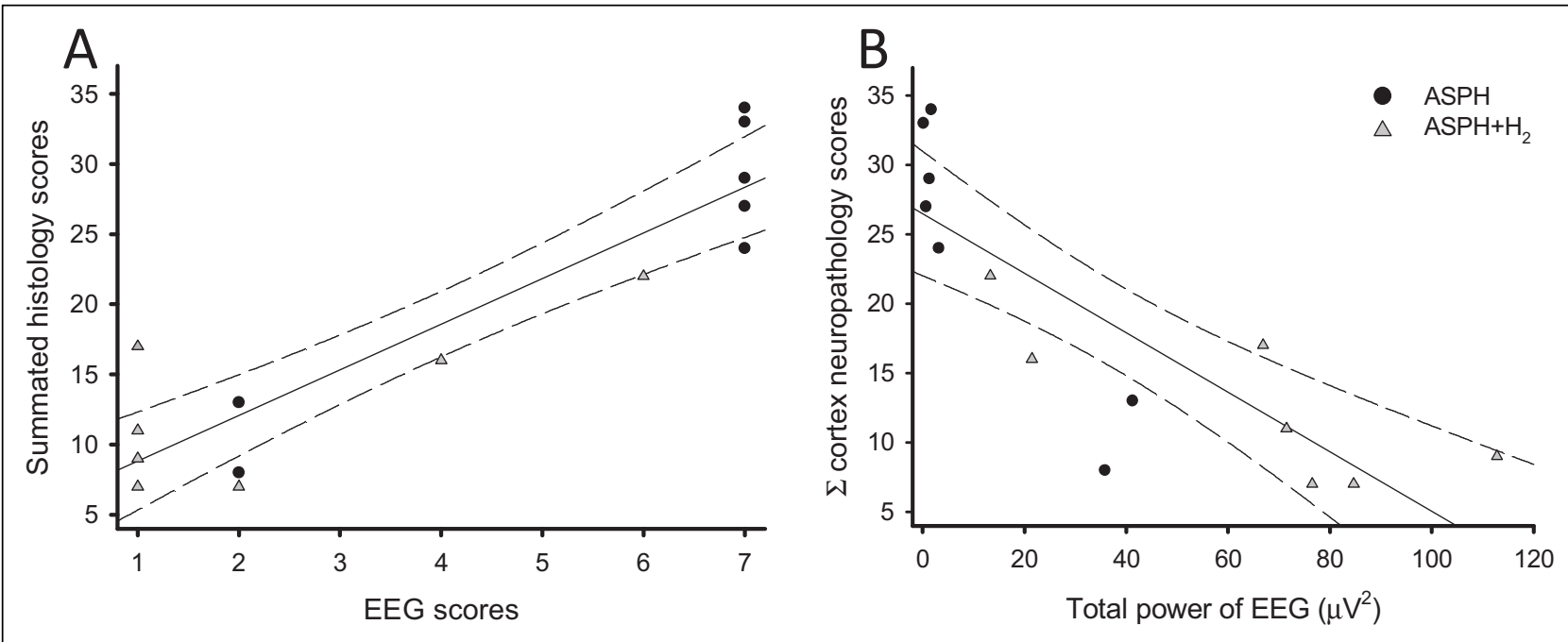

Fig. 8. EEG activity at 20 hour of survival shows good correlation with cortical neuronal damage assessed with neuropathology in the animals subjected to PA. Linear regression analysis shows significant correlations between both EEG scores (Panel A) or total EEG power (Panel B) and the sum of histopathology scores from 4 cortical regions. Solid lines represent the regression line $\left(r^{2}: 83.06 \%\right.$ of Panel A and $r^{2}: 69.90 \%$ of Panel B; $P<0.05$ ) whereas dashed lines the $95 \%$ confidence intervals. 
neuroprotection afforded by molecular $\mathrm{H}_{2}: 4 \mathrm{~h}$ inhalation of $2.1 \% \mathrm{H}_{2}$ started together with the reventilation following PA facilitated the recovery of brain electric activity, and ameliorated neuronal damage in numerous cortical and subcortical regions.

Approximately 13 million newborns require assisted ventilation at birth worldwide while 1 million newborns die annually due to PA complications (2). Despite the introduction of therapeutic hypothermia, HIE induced by PA remains a major health care problem, and translational animal models are warranted to test further putative neuroprotective therapies. PA has multiple aetiologies, and unfortunately, the onset, cause, severity and duration of PA are often difficult to determine under clinical conditions, so the exact pathophysiology of human PA is still poorly understood challenging researchers whilst designing PA/HIE animal models. The newborn piglet has become a widely accepted large animal model for studying PA/HIE as its brain developmental stage, structure and metabolism is similar to the term neonate (38). Furthermore, its size, cost and availability are also in favour of its application in PA/HIE research. During the past few decades, numerous piglet PA/HIE models were tested with variable clinical feasibility (20-25). In our previous paper assessing the effects of molecular $\mathrm{H}_{2}$ on neurovascular unit integrity after PA (19), we used 8 min tracheal tube occlusion to induce PA. This method was very useful to show delayed attenuation of cerebrovascular reactivity, however, the neuropathology revealed only limited number of irreversibly damaged neurons at 24 hour of survival. Increasing the duration of PA by tracheal occlusion to induce more severe HIE proved to be impossible because of the high occurrence of lethal cardiac complications (personal observations). There is a multitude of piglet PA models in the literature that are elicited by ventilating the animals with hypoxic gas mixtures. These models vary considerably in the applied $\mathrm{FiO}_{2}$ and whether the gas mixture contains also $\mathrm{CO}_{2}$ or not. Moreover, bilateral carotid artery occlusion is often combined with hypoxic ventilation. We chose the PA model employed in the present study based on two lines of experimental evidence. First, we found that bilateral carotid artery occlusion during normoxic or hypoxic conditions did not induce reductions in CoBF (26). Second, Helmy et al. (30) showed that in rat pups hypoxia alone $\left(9 \% \mathrm{O}_{2}\right)$ did not whereas hypoxia combined with hypercapnia $\left(9 \% \mathrm{O}_{2}\right.$ and $20 \%$ $\mathrm{CO}_{2}$ ) elicited seizures - an important hallmark of HIE. Therefore, our task was to develop a PA injury that elicits more severe HIE than our previous (tracheal occlusion) PA model $(18,19)$.

We performed a number of preliminary experiments in which we used EEG recovery as a primary indicator of HIE severity. In our previous study, none of the ASPH group animals displayed continuous (normal) EEG pattern in the first 4 hours of reventilation. We assumed therefore, that any PA stress allowing full recovery of the EEG faster than 4 hours likely induced less severe neuronal injury, and therefore more severe PA was still warranted. We first induced PA using $9 \% \mathrm{O}_{2}-20 \%$ $\mathrm{CO}_{2}$ gas mixture with increasing durations of 30,40 , and 60 mins $(\mathrm{n}=2-2-2)$ as these PA injuries were not severe enough, we reduced $\mathrm{FiO}_{2}$ to $6 \%$ and performed additional experiments with 35,25 , and 15 min duration ( $n=2,2$, and 3 , respectively). Animals subjected to $35-25$ min PA were presented with isoelectric/very low voltage EEG at $24 \mathrm{~h}$ survival and were all dependent on inotropics to keep their blood pressure at $40-45$ $\mathrm{mmHg}$ indicating cardiac complications. In contrast, all three animals subjected to 15 min of PA had quickly recovered their electrical brain activity. Our experiments using PA lasting for 20 min produced consistently animals that showed both stable hemodynamics and EEG signs of moderate/severe brain injury forming later group ASPH of the present study. We would like to note that $\mathrm{pH}$ and blood gas values taken at the last minute of PA using a given gas mixture were very similar to each other indicating that in this model likely not the absolute degree of hypoxia, hypercapnia or acidosis itself rather than its duration determines the degree of elicited brain damage. However, hypercapnia appears to be an important determinant of HIE severity. Goodwin et al. (39) reported that newborns with $\mathrm{P}_{\mathrm{a}} \mathrm{CO}_{2}$ values greater than $140 \mathrm{mmHg}$, manifested major neurological deficits and good correlation to seizure activity. Moreover, in an other study by Belai et al. (40), HIE did not develop in all infants with $\mathrm{P}_{\mathrm{a}} \mathrm{CO}_{2}$ below $140 \mathrm{mmHg}$. A marked advantage of our current model is also the stability of systemic hemodynamic parameters, virtually no animals were lost during the PA/immediate reventilation period and none of the animals required pharmacological treatment for hypotension afterwards. This is in sharp contrast with the PA model published by Chalak et al. (41), who ventilated the animals with $5.3 \% \mathrm{O}_{2}$ and $7.5 \%$ $\mathrm{CO}_{2}$ gas mixture and decreased the RR by $101 /$ min every 15 minute and reached asystole in order to produce blood gas and $\mathrm{pH}$ values that are similar to our results and in accordance with those reported from term newborns undergoing severe PA (40, 42). We found a moderate increase in CoBF during PA in the present study, so our PA model clearly does not induce cortical ischemia, however, the increase in CoBF was still $2-3$ times less $(\sim 35 \%$ versus $\sim 100 \%)$ than in animals ventilated with the $10 \% \mathrm{O}_{2}$ combined with bilateral carotid artery occlusion (26). As we previously reported (43) that 10 minute hydrogen ventilation did not influence cortical microcirculation in newborn piglets, hence we did not test the effect of the neuroprotectant in a separate group in the present study.

Unfortunately, in the clinical setting precise prediction of neurodevelopmental outcome in infants affected by PA/HIE is difficult in most cases. A recent meta-analysis suggested that neurophysiology tests (i.e. serial EEG-s) within the first week of life may prove to be promising tools in this effort (44). Moreover, magnetic resonance spectroscopy (MRS) and imaging (MRI) offer an invaluable aid for assessing morphologic and metabolic changes in the neonatal brain as early as the first days of life (45) but its accessibility, the facility to serial imaging as well as the necessary human and material resources are not available everywhere. Bedside neonatal EEG monitoring provides continuous information about the impaired cortical electrical activity after PA and was reported to be a useful tool to predict late outcome as early as 24 hours $(46,47)$. Although, it is widespread in neonatal intensive care as its application and maintenance does not require special conditions, however, trained staff is required to interpret the EEG patterns. The clinical evaluation of EEG signals is predominantly based on the amplitudes of waves, the presence/absence of sleep-wake cycles, seizures, repetitive signals; the quantification of given periods is more challenging. Consequently, custom-made scoring scales were introduced and were widely accepted in the literature (48), thus first, we established our own amplitude-based scoring system according to international recommendations (34), but we also simultaneously determined the total power of the recorded EEG at given time points. Our PA model elicited similar, severe electrocortical depression within $1-2$ minutes in groups ASPH and $\mathrm{ASPH}+\mathrm{H}_{2}$ that remained practically isoelectric $(<5 \mu \mathrm{V}$, high EEG scores, low total power) in the asphyxia-only animals whereas EEG activity was at least partially restored in the hydrogen treated group. Interestingly, EEG scores and the total power of EEG of all asphyxiated animals (groups ASPH and $\mathrm{ASPH}+\mathrm{H}_{2}$ ) at 20 hours showed strong correlation (Fig. 8., $\mathrm{r}^{2}$ : $83.06 \%$ and $69.9 \%$; respectively, $\mathrm{P}<0.05)$ to the summated histopathological scores characterizing cortical brain damage, thus EEG activity this time point might be predictive for indicating the severity of brain injury in our present model. Indeed, the recovery of EEG amplitudes correlated well with the preservation of the cerebral cortex after hydrogen ventilation. 
Neuronal injury was assessed by a previously reported scoring system as cell counting alone does not represent the pattern of cortical damage: a single panlaminar necrosis may elicit more severe clinical outcome compared to similar number of injured neurons distributed along the 20 fields of vision. Concerning the other brain regions studied, it is worth noting that the hippocampal CA1 and CA3 regions as well as the basal ganglia suffered the most extensive neuronal damage in our model in accordance with early MRI findings in PA/HIE survivors (49), and that $\mathrm{H}_{2}$ could exert marked neuroprotective effect in these areas as well. Despite marked neuronal injury, we could not detect increases in plasma NSE levels after asphyxia. This finding is in accordance with those of Nagdyman et al. (50) who reported also unaltered NSE levels in asphyxiated term newborns during the first day of life. Admittedly, Kecskes et al. (51) reported NSE elevations in piglets at 24 hours, but only following a longer PA stress (40 min hypoxic ventilation combined with arterial hypotension) that likely severely affected at least transiently the integrity of the blood brain barrier.

Therapeutic hypothermia has become the gold standard care to combat HIE development (8), however, not all of the babies may benefit from total body cooling (52) thus supplementary neuroprotective efforts are urgently required. Furthermore, 'therapeutic' hypothermia has recently been reported not to prevent white matter damage following PA in piglets, but hypothermia was in fact shown to per se induce white matter damage in normoxic controls, raising concerns about the safety of this approach (53). Molecular hydrogen was introduced as a novel hydroxyl radical scavenger by Ohsawa et al. (13) and since then, its antioxidant, anti-inflammatory and anti-apoptotic effects were proved in numerous preclinical and clinical studies (14). Among the animal models of PA/HIE, first Cai et al. (15) reported neurological improvement after molecular hydrogen $\left(2 \% \mathrm{H}_{2}\right.$ in medical air) resuscitation in a neonatal rodent HIE model. This finding was in accordance with the neuroprotective effect of $\mathrm{H}_{2}$ in a transient global ischemia rat model showing improved neuronal survival $\left(2 \% \mathrm{H}_{2}\right.$ in medical air) and cognitive functions in rats (54). Surprisingly, Matchett et al. (17) reported no benefit from $\mathrm{H}_{2}$ administration in a neonatal rat HIE model, in contrast, $\mathrm{H}_{2}$ pretreatment even appeared to enhance hypoxic/ischemic brain damage. To the best of our knowledge, our research group was the first who reported the beneficial effects of hydrogen in a large animal PA/HIE model $(18,19)$. The current study importantly expands our previous knowledge in that aspect that $\mathrm{H}_{2}$ is not only able to reduce injury in a mild but also in a severe PA/HIE model. The effect of $\mathrm{H}_{2}$ is quite robust in a translationally feasible administration route: administration started after the PA together with the reventilation efforts. Furthermore, we showed in the present study for the first time in this large animal model that molecular hydrogen alleviated the level of oxidative stress, indicated by reduced $8-\mathrm{OHdG}$ levels in the parietal cortex, similar to method and findings of Zhai et al. (55) who reported the antioxidant capacity of hydrogen in an adult stroke rat model.

The direct molecular target/ mechanism of action of this relatively inert gas is still unknown, although several biochemical markers have shown altered expression followed by hydrogen administration (56). However, it is often difficult, or virtually impossible, to decipher in vivo if the observed effect has a causative role in hydrogen-induced neuroprotection or rather is the consequence of the hydrogen-induced protection achieved through an independent mechanism. Nevertheless, very recently hypoxia-induced elevations in cerebrocortical interleukin $18 \mathrm{mRNA}$ levels have been shown to be inhibited by ventilation with $2 \% \mathrm{H}_{2}$ in piglets (57) suggesting that in addition to the putative reactive oxygen species scavenging capacity, anti-inflammatory effect of hydrogen can contribute to reduced neuronal death after PA in the newborn brain.
Molecular hydrogen was administered as before in our current model for two reasons. First, the brevity of the therapeutic window demands the introduction of a potential neuroprotective intervention up to 6 hours, secondly, reventilation/reperfusion-induced ROS generation (58) is the most important contributor to hypoxic-ischemic brain damage $(59,60)$. Hydrogen resuscitation facilitated the recovery of the EEG amplitudes in our model, moreover, prevented structural neuronal damage assessed at 24 hour of survival.

The present study has some limitations. Although this 24 hour observation period likely spans the time frame in which molecular hydrogen can afford protection, it does not allow conclusions on long-term neuroprotection or late neurodevelopmental outcome with certainty. A longer, at least 48 hour survival would likely allow us to study if anti-apoptotic mechanisms played also a role in the neuroprotective effect of molecular $\mathrm{H}_{2}$, using for instance cleaved caspase 3 immunohistochemistry that has already been shown in piglets to indicate anti-apoptotic effects elicited by hypothermia or melatonin at this timepoint $(61,62)$. Moreover, 48 hours survival would also allow us to study altered neurotoxic activation of microglia by determining microglial ramification index or CD86 mRNA levels that have also been found useful to characterize anti-inflammatory neuroprotection in this animal model $(61,62)$. Second, the neuroprotective effect of molecular hydrogen in combination with therapeutic hypothermia was not tested, although the latter became the gold standard care after PA. Unfortunately, the use of hypothermia with this survival period was not feasible; although cooling can be achieved within an hour, rewarming must be not faster than $0.5^{\circ} \mathrm{C} / \mathrm{h}$ (53) that would last more than 10 hours, allowing only very limited time in the therapeutic temperature range likely causing underestimation of its neuroprotective potential. As molecular hydrogen was found in the present study to be effective against moderate/severe HIE stress, combining the therapeutic effect of molecular hydrogen with total body hypothermia using an even longer ( $48-72$ hours) survival period remains our future direction. The third limitation is the use of anaesthesia/analgesia during the experiments, especially during PA complicating translation of the model and the results to the human clinical situation. Adequate anaesthesia is mandatory to meet ethical concerns using laboratory animals, however, we carefully chose an anaesthethic/analgesic regimen that is often used in neonatology (63) to support HIE patients: morphine to combat mechanical ventilation-induced pain and stress (64), and midazolam is routinely administered to suppress neonatal seizure activity. Furthermore, midazolam has been shown to unaffect the EEG and not jeopardising amplitude-based EEG analysis (65).

In conclusion, we introduce a new piglet PA/HIE model that faithfully represents the human clinical and metabolic hallmarks of PA and results in moderate/severe brain injury. The degree of HIE has been characterized by depressed EEG activity and robust histopathology findings at 24 hour of survival confirming a more severe asphyxic insult compared to our previous PA model (tracheal occlusion) (19). Molecular hydrogen administered in this severe PA/HIE model displays marked neuroprotection shown by facilitating the restoration of brain electrical activity and preserving structural integrity virtually in all assessed brain regions.

Authors' contributions: Janos Nemeth: performing in vivo experiments, EEG scoring, neuropathology evaluation, writing the manuscript; Valeria Toth-Szuki: performing in vivo experiments, histology; Viktoria Varga: performing in vivo experiments, neuropathology evaluation; Viktoria Kovacs: performing in vivo experiments; Gabor Remzso: performing in vivo experiments, EEG scoring; Ferenc Domoki: performing in vivo experiments, statistics, writing the manuscript. 
Acknowledgements: We gratefully acknowledge that the EEG device used in the study was provided by Prof. Kai Kaila, Neuroscience Center, University of Helsinki, Helsinki, Finland.

The study was supported by grants from the National Scientific Research Fund of Hungary (OTKA K100851) and from the Hungarian Brain Research Program (KTIA_13_NAPA-I/13). Ferenc Domoki was supported by the Janos Bolyai Research Scholarship of the Hungarian Academy of Sciences. Janos Nemeth was supported by the 'Nemzeti Tehetseg Program' of the 'Emberi Eroforras Tamogataskezelo' from the Hungarian Ministry of Human Capacities.

Conflict of interests: None declared.

\section{REFERENCES}

1. Liu, L., Oza S, Hogan D, et al. Global, regional, and national causes of child mortality in 2000-13, with projections to inform post-2015 priorities: an updated systematic analysis. Lancet 2015 ; 385: 430-440.

2. Vali P, Mathew B, Lakshminrusimha S. Neonatal resuscitation: evolving strategies. Matern Health Neonatol Perinatol 2015; 1: 4. doi: 10.1186/s40748-014-0003-0.

3. Lawn J, Shibuya K, Stein C. No cry at birth: global estimates of intrapartum stillbirths and intrapartum-related neonatal deaths. Bull World Health Organ 2005; 83: 409-417.

4. Herrera-Marschitz M, Morales P, Leyton L, et al. Perinatal asphyxia: current status and approaches towards neuroprotective strategies, with focus on sentinel proteins. Neurotox Res 2011; 19: 603-627.

5. Low JA. Intrapartum fetal asphyxia: definition, diagnosis, and classification. Am J Obstet Gynecol 1997; 176: 957-959.

6. Volpe JJ. Neonatal encephalopathy: an inadequate term for hypoxic-ischemic encephalopathy. Ann Neurol 2012; 72: 156-166.

7. Eunson P. The long-term health, social, and financial burden of hypoxic-ischaemic encephalopathy. Dev Med Child Neurol 2015; 57 (Suppl. 3): 48-50.

8. Perlman JM, Wyllie J, Kattwinkel J, et al. Part 11: Neonatal resuscitation: 2010 International Consensus on Cardiopulmonary Resuscitation and Emergency Cardiovascular Care Science With Treatment Recommendations. Circulation 2010; 122 (16 Suppl. 2): S516-S538.

9. Azzopardi DV, Strohm B, Edwards AD, et al. Moderate hypothermia to treat perinatal asphyxial encephalopathy. N Engl J Med 2009; 361: 1349-1358.

10. Thoresen M. Who should we cool after perinatal asphyxia? Semin Fetal Neonatal Med 2015; 20: 66-71.

11. Azzopardi D, Brocklehurst P, Edwards D, et al. The TOBY Study. Whole body hypothermia for the treatment of perinatal asphyxial encephalopathy: a randomised controlled trial. BMC Pediatr 2008; 8: 17. doi: 10.1186/1471-2431-8-17.

12. Roohey T, Raju TN, Moustogiannis AN. Animal models for the study of perinatal hypoxic-ischemic encephalopathy: a critical analysis. Early Hum Dev 1997; 47: 115-146.

13. Ohsawa I, Ishikawa M, Takahashi $\mathrm{K}$, et al. Hydrogen acts as a therapeutic antioxidant by selectively reducing cytotoxic oxygen radicals. Nat Med 2007; 13: 688-694.

14. Ohta S. Molecular hydrogen as a novel antioxidant: overview of the advantages of hydrogen for medical applications. Methods Enzymol 2015; 555: 289-317.

15. Cai J, Kang Z, Liu WW, et al. Hydrogen therapy reduces apoptosis in neonatal hypoxia-ischemia rat model. Neurosci Lett 2008; 441: 167-172.
16. Cai J, Kang Z, Liu K, et al. Neuroprotective effects of hydrogen saline in neonatal hypoxia-ischemia rat model. Brain Res 2009; 1256: 129-137.

17. Matchett GA, Fathali N, Hasegawa Y, et al. Hydrogen gas is ineffective in moderate and severe neonatal hypoxiaischemia rat models. Brain Res 2009; 1259: 90-97.

18. Domoki F, Olah O, Zimmermann A, et al. Hydrogen is neuroprotective and preserves cerebrovascular reactivity in asphyxiated newborn pigs. Pediatr Res 2010; 68: 387-392.

19. Olah O, Toth-Szuki V, Temesvari P, Bari F, Domoki F. Delayed neurovascular dysfunction is alleviated by hydrogen in asphyxiated newborn pigs. Neonatology 2013; 104: 79-86.

20. Temesvari P, Joo F, Koltai M, et al. Cerebroprotective effect of dexamethasone by increasing the tolerance to hypoxia and preventing brain oedema in newborn piglets with experimental pneumothorax. Neurosci Lett 1984; 49: 87-92.

21. Pourcyrous M, Leffler C, Busija D. Postasphyxial increases in prostanoids in cerebrospinal fluid of piglets. Pediatr Res 1988; 24: 229-232.

22. Armstead WM, Mirro R, Busija DW, Leffler CW. Postischemic generation of superoxide anion by newborn pig brain. Am J Physiol 1988; 255: H401-H403.

23. Martin LJ, Brambrink A, Koehler RC, Traystman RJ. Primary sensory and forebrain motor systems in the newborn brain are preferentially damaged by hypoxia-ischemia. J Comp Neurol 1997; 377: 262-285.

24. Lorek A, Cady EB, Wyatt JS, et al. Delayed ('secondary') cerebral energy failure after acute hypoxia-ischemia in the newborn piglet: continuous 48-hour studies by phosphorus magnetic resonance spectroscopy. Pediatr Res 1994; 36: 699-706.

25. Wang JM, Zhang GF, Zhou WH, Jiang ZD, Shao XM. Changes in amplitude-integrated electroencephalograms in piglets during selective mild head cooling after hypoxiaischemia. Pediatr Neonatol 2014; 55: 282-290.

26. Domoki F, Zolei-Szenasi D, Olah O, et al. Comparison of cerebrocortical microvascular effects of different hypoxicischemic insults in piglets: a laser-speckle imaging study. J Physiol Pharmacol 2014; 65: 551-558.

27. Haaland K, Orderud WJ, Thoresen W. The piglet as a model for cerebral circulation: an angiographic study. Biol Neonate 1995; 68: 75-80

28. Sakr HF, Khalil KI, Hussein AM, Zaki MS, Eid RA, Alkhateeb M. Effect of dehydroepiandrosterone (DHEA) on memory and brain derived neurotrophic factor (BDNF) in a rat model of vascular dementia. J Physiol Pharmacol 2014; 65: 41-53.

29. Kovalska, M., Kovalska L, Mikuskova K, Adamkov M, Tatarkova Z, Lehotsky J. p-ERK involvement in the neuroprotection exerted by ischemic preconditioning in rat hippocampus subjected to four vessel occlusion. J Physiol Pharmacol 2014; 65: 767-776.

30. Helmy MM, Tolner EA, Vanhatalo S, Voipio J, Kaila K. Brain alkalosis causes birth asphyxia seizures, suggesting therapeutic strategy. Ann Neurol 2011; 69: 493-500.

31. Hall RW, Boyle E, Young T. Do ventilated neonates require pain management? Semin Perinatol 2007; 31: 289-297.

32. Laptook AR, Stonestreet BS, Oh W. The effect of carotid artery ligation on brain blood flow in newborn piglets. Brain Res 1983; 276: 51-54.

33. van Rooij LG, van den Broek MP, Rademaker CM, de Vries LS. Clinical management of seizures in newborns : diagnosis and treatment. Paediatr Drugs 2013; 15: 9-18.

34. Tsuchida TN, Wusthoff CJ, Shellhaas RA, et al. American clinical neurophysiology society standardized EEG terminology and categorization for the description of continuous EEG monitoring in neonates: report of the 
American Clinical Neurophysiology Society Critical Care Monitoring Committee. J Clin Neurophysiol 2013; 30: 161-173.

35. Foster KA, Colditz PB, Lingwood BE, Burke C, Dunster KR, Roberts MS. An improved survival model of hypoxia/ischaemia in the piglet suitable for neuroprotection studies. Brain Res 2001; 919: 122-131.

36. Busija DW, Leffler CW, Wagerle LC. Responses of newborn pig pial arteries to sympathetic nervous stimulation and exogenous norepinephrine. Pediatr Res 1985; 19: 1210-1214.

37. Randall GC. pH values and blood-gas tensions in the normal piglet during the first 48 hours of life. Biol Neonate 1972; 20: 68-73.

38. Dickerson JW, Dobbing J. Prenatal and postnatal growth and development of the central nervous system of the pig. Proc $R$ Soc Lond B Biol Sci 1967; 166: 384-395.

39. Goodwin TM, Belai I, Hernandez P, Durand M, Paul RH. Asphyxial complications in the term newborn with severe umbilical acidemia. Am J Obstet Gynecol 1992; 167: 15061512.

40. Belai Y, Goodwin TM, Durand M, Greenspoon JS, Paul RH, Walther FJ. Umbilical arteriovenous $\mathrm{pO}_{2}$ and $\mathrm{pCO}_{2}$ differences and neonatal morbidity in term infants with severe acidosis. Am J Obstet Gynecol 1998; 178: 13-19.

41. Chalak LF, Barber CA, Hynan L, Garcia D, Christie L, Wyckoff $\mathrm{MH}$. End-tidal $\mathrm{CO} 2$ detection of an audible heart rate during neonatal cardiopulmonary resuscitation after asystole in asphyxiated piglets. Pediatr Res 2011; 69: 401-405.

42. Engle WD, Laptook AR, Perlman JM. Acute changes in arterial carbon dioxide tension and acid-base status and early neurologic characteristics in term infants following perinatal asphyxia. Resuscitation 1999; 42: 11-17.

43. Domoki F, Zolei D, Olah O, et al. Evaluation of laser-speckle contrast image analysis techniques in the cortical microcirculation of piglets. Microvasc Res 2012; 83: 311-317.

44. van Laerhoven $\mathrm{H}$, de Haan TR, Offringa M, Post B, van der Lee JH. Prognostic tests in term neonates with hypoxicischemic encephalopathy: a systematic review. Pediatrics 2013; 131: 88-98.

45. Rutherford MA, Pennock JM, Schwieso JE, Cowan FM, Dubowitz LM. Hypoxic ischaemic encephalopathy: early magnetic resonance imaging findings and their evolution. Neuropediatrics 1995; 26: 183-191.

46. Sinclair DB, Campbell M, Byrne P, Prasertsom W, Robertson CM.EEG and long-term outcome of term infants with neonatal hypoxic-ischemic encephalopathy. Clin Neurophysiol 1999; 110: 655-659.

47. Pezzani C, Radvanyi-Bouvet MF, Relier JP, Monod N. Neonatal electroencephalography during the first twentyfour hours of life in full-term newborn infants. Neuropediatrics 1986; 17: 11-18.

48. Agnew DM, Koehler RC, Guerguerian AM. Hypothermia for 24 hours after asphyxic cardiac arrest in piglets provides striatal neuroprotection that is sustained 10 days after rewarming. Pediatr Res 2003; 54: 253-262.

49. Rutherford MA, Pennock JM, Counsell SJ, et al. Abnormal magnetic resonance signal in the internal capsule predicts poor neurodevelopmental outcome in infants with hypoxicischemic encephalopathy. Pediatrics 1998; 102: 323-328.

50. Nagdyman N, Komen W, Ko HK, Muller C, Obladen M. Early biochemical indicators of hypoxic-ischemic encephalopathy after birth asphyxia. Pediatr Res 2001; 49: 502-506.
51. Kecskes Z, Dunster KR, Colditz PB. NSE and S100 after hypoxia in the newborn pig. Pediatr Res 2005; 58: 953-957.

52. Sarkar S, Bhagat I, Dechert RE, Barks JD. Predicting death despite therapeutic hypothermia in infants with hypoxicischaemic encephalopathy. Arch Dis Child Fetal Neonatal Ed 2010; 95: F423-F428.

53. Wang B, Armstrong JS, Reyes M, et al. White matter apoptosis is increased by delayed hypothermia and rewarming in a neonatal piglet model of hypoxic ischemic encephalopathy. Neuroscience 2016; 316: 296-310.

54. Ge P, Zhao J, Li S, Ding Y, Yang F, Luo Y. Inhalation of hydrogen gas attenuates cognitive impairment in transient cerebral ischemia via inhibition of oxidative stress. Neurol Res 2012; 34: 187-194.

55. Zhai X, Chen X, Shi J, et al. Lactulose ameliorates cerebral ischemia-reperfusion injury in rats by inducing hydrogen by activating Nrf2 expression. Free Radic Biol Med 2013; 65: 731-741.

56. Hong Y, Chen S, Zhang JM. Hydrogen as a selective antioxidant: a review of clinical and experimental studies. J Int Med Res 2010; 38: 1893-1903.

57. Pankratov L, et al. Resuscitation with hydrogen gas inhibits gene expression of IL-18 after severe asphyxia in a newborn pig model. In: Brain 2015, Vancouver, Canada, 2015.

58. Korbecki J, Baranowska-Bosiacka I, Gutowska I, Chlubek D. The effect of reactive oxygen species on the synthesis of prostanoids from arachidonic acid. J Physiol Pharmacol 2013; 64: 409-421.

59. Gunn AJ. Cerebral hypothermia for prevention of brain injury following perinatal asphyxia. Curr Opin Pediatr 2000; 12: 111-115.

60. Thoresen M, Tooley J, Liu X, et al. Time is brain: starting therapeutic hypothermia within three hours after birth improves motor outcome in asphyxiated newborns. Neonatology 2013; 104: 228-233.

61. Robertson NJ, Faulkner S, Fleiss B, et al. Melatonin augments hypothermic neuroprotection in a perinatal asphyxia model. Brain 2013; 136: 90-105.

62. Alonso-Alconada D, Broad KD, Bainbridge A, et al. Brain cell death is reduced with cooling by 3.5 degrees $\mathrm{C}$ to 5 degrees $\mathrm{C}$ but increased with cooling by 8.5 degrees $\mathrm{C}$ in a piglet asphyxia model. Stroke 2015; 46: 275-278.

63. Hall RW, Shbarou RM. Drugs of choice for sedation and analgesia in the neonatal ICU. Clin Perinatol 2009; 36: 215-226.

64. Aranda JV, Carlo W, Hummel P, Thomas R, Lehr VT, Anand $\mathrm{KJ}$. Analgesia and sedation during mechanical ventilation in neonates. Clin Ther 2005; 27: 877-899.

65. Olischar M, Davidson AJ, Lee KJ, Hunt RW. Effects of morphine and midazolam on sleep-wake cycling in amplitude-integrated electroencephalography in postsurgical neonates $\geq 32$ weeks of gestational age. Neonatology 2012; 101: 293-300.

Received: April 28, 2016

Accepted: October 28, 2016

Author's address: Dr. Ferenc Domoki, Department of Physiology, University of Szeged, School of Medicine, 10 Dóm tér, Szeged, 6720, Hungary.

E-mail: domoki.ferenc@med.u-szeged.hu 